\title{
38118
}

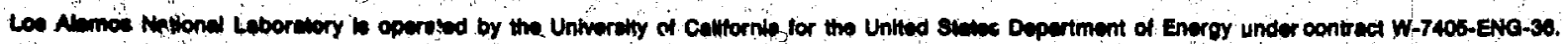




\title{
Measurements of Threshold Behavior for One- and Two-Electron Photodetachment from the $\mathrm{H}^{-}$lon
}

\author{
Charles Alan Frost *
}

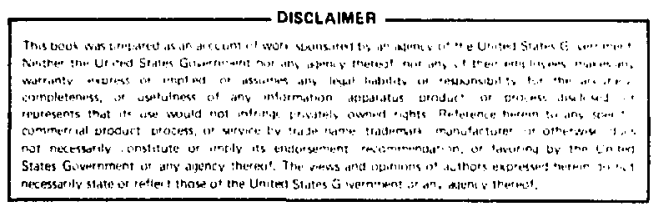

*Sandia Laboratories, P. O. Box 5800, Albuquerque, NM 87185.
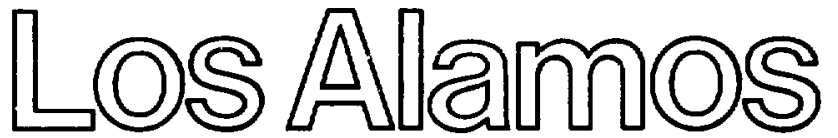

Los Alamos National Laboratory

Los Alamos,New Mexico 87545 
TABLE OF CONTENTS

Page

List of figures . . . . . . . . . xi

List of Tables . . . . . . . . . $x v$

I. Introdućtion . . . . . . . . . 1

A. Motivation and Background ..... 1

B. Calculations and Measurements of Photodetachment Cross Section . . . 4

C. Experimental Method . . . . . . 18

II. Theoretical Background . . . . . . . 24

A. Photodetachment . . . . . . . 24

B. One-Electron Threshold ..... . 29

C. Two-Electron Threshold . . . . . 32

III. Experiment . . . . . . . . . . 41

A. Experimental Setup ........ 41

B. Physical Arrangement . . . . . . 44

C. $\mathrm{H}^{-}$Beam Characteristics . . . . . 54

D. Laser and Optics . . . . . . . 58

E. Magnet Configurations . . . . . 67

F. Sensors . . . . . . . . . 78

G. Elsctronics . . . . . . . . 89

H. Signal Digitization . . . . . . . 98

I. Silicon Detector Electronics . . . 104

J. Timing Circuits . . . . . . 110

K. Computer Units . . . . . . . . 118 
L. Data Analysis Code . . . . . . . 122

M. Calibration Procedures . . . . . 125

IV. Results and Analysis . . . . . . . 133

A. Analysis Procedure . . . . . . 133

B. One-Electron Photodetachment . . . 139

C. One-Electron Threshold . . . . . 155

D. Magnetic Field Stripping . . . . . 171

E. Two-Electron Photoejection . . . . 186

F. Two-Electron Threshold . . . . . 198

V. Conclusion . . . . . . . . . . 221

Acknowledgements . . . . . . . . 224

References............ 225 


\section{LIST OF FICURES}

Figure

Page

1 Photodetachment Cross Section of $\mathrm{H}^{-}$From J. T. Broad and W. P. Reinhardt . . .

2 Experimental Photodetachment Relative Cross Section of Smith and Burch. After Smith and Burch . . . . . . . . . .

$3 \mathrm{H}^{-}$Photodetachment Cross Section in the

Resonance Region From J. T. Broad and

W. P. Reinhardt . . . . . . . . .

4 Two-Electron Cross Section Calculated by Broad and Reinhardt . . . . . . .

5 Comparison of One and Two-Electron Cross Sections as Calculated by Broad and Reinhardt . . . . . . . . . . .

6 Center of Mass Photon Energies Produced by Doppler Shifting Various Laser Lines at LAMPF $(\beta=0.842)$. . . . . . .

7 Schematic of Apparatus . . . . .

8 Physical Arrangement . . . . . . .

9 Photograph of Apparatus Showing Beam Plpe . . . . . . . . . . . . .

10 Section View of Chamber Showing Rotating Mirror Arrangement (Spider) . . . . .

11 Photograph of Scattering Chamber . .

12 Schematic View of Rotating Mirror Arrangement (Spider) . . . . . . . . . .

13 The $\mathrm{H}^{-}$and Laser Beam Structure . . . 57

14 Laser Block Diagram . . . . . . . . 60

15 Optical Bench Photograph . . . . . . 66

16 Magnet and Particle Detector Geometry . 69

17 Sweep Magnets Photograph . . . . . . 76 
18 Photograph of Scintillators .....

19 Bets Particles Range in Silicon. After ORTEC Instruction Manual for Surface Barrier Detectors . . . . . . .

20 Photograph of Part of Fast Electronfes Setup . . . . . . . . . . . . .

21 Electronics Block Diagram . . . . . 9:-

22 Electronics Control Diagram . . . . . 96

23 Signal Digitization Simplified Schematic 100

24 Silicon Detector Circuit . . . . . . 106

25 Timing Diagram ......... 111

26 Laser Trigger Generator Logic Diagram • 116

27 Computer Control Block Diagram . . . 120

28 Feshbach Scan for Calibration . . . . 130

29 Pulse Height Spectrum from Particle Detector . . . . . . . . . . 132

30 One-Electron Cross Section Compared to the Theory of Armstrong . . . . . .

31 One-Electron Cross Section Normalized to the Theory of Broad and Reinhardt . 145

32 Cross Sections from 10.0 to $15.6 \mathrm{eV}$ Raw Form . . . . . . . . . 146

33 Cross Section from $e^{-}$Detector Coarse Scar . . . . . . . . . . . 148

34 One-Elegtron Cross Section Coarse Scan (From $\mathrm{H}^{\mathrm{D}}$ Detector) ........... 150

35 One-Electron Cross Section Normalized to Broad and Reinhardt . . . . . . . 152

36 One-Eiectron Cross Section Global Scan 154

37 Threshold for One-Electron Photodetachment ............. 158 
38 One-Electron Threshold Fine Scan ...

39 Plot of $\mathrm{X}^{2}$ Versus $\mathrm{N}$. . . . . . . . 164

40 One-Elnctron Threshold Restricted

Energy Range . . . . . . . . . .

41 Plot of $N$ Versus Energy Range . . .

42 Cross Section from $\mathrm{H}^{+}$Detector with a Stripping Field of 3600 Gauss ... .

43 Cross Section From $\mathrm{H}^{+}$Detector Fine Scan Near $n=5$ Threshold with 3600 Gauss Field . . . . . . . . . . . .

44 Cross Section from $\mathrm{H}^{+}$Detector with 2200 Gauss Field ... . . . . . . .

45 Fine Scan of Cross Section Calculated with $\mathrm{H}^{+}$Detector Signal ( $B=2200$ Gauss)

46 Fine Scan B=780 Gauss . . . . . . . 182

47 Fine Scan B=380 Gauss . . . . . . 185

48 Two-Elepctron Photoejection Cross Section from $\mathrm{H}^{+}$Detector ......... .

49 Ratio of $\mathrm{H}^{+}$Signal to $\mathrm{H}^{\circ}$ Signal ...

50 Two-Electron Cross Section Normalized to the One-Electron Cross Section Calculation of Broad and Reinhardt ....

51 Fine Scan Oyer the Two-Electron Threshold Using the $\mathrm{H}^{+}$Detector . . . . . .

52 Fine Scan Over the Tyo-Electron Threshold Using the Ratio of $\mathrm{H}^{+}$to $\mathrm{H}^{\delta}$ Signals.

203

53 Two-Elęctron Cross Section Calculated from $\mathrm{H}^{+}$Detector Using Points Above $14.35 \mathrm{eV}$ Only ........... .

54 Ratio of $\mathrm{H}^{+}$to $\mathrm{H}^{\circ}$ Signal Above $14.35 \mathrm{eV} 208$

55 Curve Fit with Separate Exponents in the Two Regions 
56 Temkin's Form Fit to Data from $\mathrm{H}^{+}$ Detector ..........

57 Temkjn's Form Fit to Data from $\mathrm{H}^{+} / \mathrm{H}^{\circ} \cdot \ldots . . . . . . . . . .$.

58 Fit Showing Oscillations ......

218 


\section{LIST OF TABLES}

Table

Page

1. Relative $\mathrm{H}^{-}$Photodetachment Cross

Section after Smith and Burch . . .

2. $\mathrm{H}^{-}$Photodetachment Cross Section

Calculated by Broad and Reinhardt as

a Function of Warelength ...... .

3. Specifications for Quanta-Ray DCR 1 Laser . . . . . . . . . . . .

4. Magnet Configurations . . . . . . 74

5. Energy of the Ground State of $\mathrm{H}^{-}$. . 166

6. Typical Results of Fitting Temkin's Form 
MEASUREMENTS OF THRESHOLD BEHAVIOR FOR ONE- AND

TWO-ELECTRON PHOTODETACHMENT FROM THE H${ }^{-}$ION

\section{Charles Alan Frost \\ B.S., Physics, Auburn University, 1969}

ABSTRACT

One and two-electron photodetachment from the $\mathrm{H}^{-}$ion by a single photon has been studied using a crossed beam apparatus. A Q-switched laser beam was directed across the $800 \mathrm{MeV} \mathrm{H}^{-}$beam at LAMPF ( $\beta=0.842$ ) resulting in Doppler-shifted photon energies in the $\mathrm{H}^{-}$barycentric frame, which were tunable from $0.4 \mathrm{eV}$ to $15.5 \mathrm{eV}$ by changing the intersection angle. The particles $\left(e^{-}, \mathrm{H}^{\circ}\right.$, $\mathrm{H}^{+}$) resulting from photodetachment reactions were magnetically deflected into scintillation detectors allowing the total and partial cross sections for $1 e^{-}$and $2 e^{-}$processes to be separately measured. The "2 $\mathrm{e}^{-1}$ signal $\left(\mathrm{H}^{+}\right)$was produced by two different mechanisms the true signal

$$
\mathrm{Y}+\mathrm{H}^{-}+\mathrm{H}^{+}+2 \mathrm{e}^{-}
$$

and the background process

$$
\gamma+\mathrm{H}^{-}+\mathrm{H}^{\circ}(\mathrm{n}) \text {, followed by motional electric field }
$$

ionization of excited $\mathrm{H}^{\circ}$.

Two-electron photodetachment which had been predicted theoretically was observed for the first time, and the relative cross section was measured from threshold to 15.5 
$e V$. The $1 e^{-}$cross section was measured simultaneously allowing normalization of the $2 \mathrm{e}^{-}$datia to the calculated $1 \mathrm{e}^{-}$cross section. The results were compared with the 1976 calculation of Broad and Relnhardt and found to agree within the combined uncertainty of theory and experiment. The $2 e^{-}$threshold region was examined in detail, and the data were compared with theory.

The $1 e^{-}$cross section was measured from threshold to $15.6 \mathrm{eV}$ and compared to theory with qualitative agreement. The $1 e^{-}$threshold region was examined in detail. The data were compared with theory and found to be consistent with the predicted $3 / 2$ power threshold law. The range of the law was investigated. In the course of this work the electron affinity of hydrogen was determined to be $0.753 \mathrm{eV}$ $\pm 0.005 \mathrm{eV}$ in agreement with theory.

Partial cross sections into excited states of $\mathrm{H}^{\circ}$ were measured for the first time using the field ionization effect. The threshold for excitation into the $\mathrm{H}^{\circ} \quad(n=5)$ state was clearly observed. Thresholds for channels into $\mathrm{H}^{\mathrm{O}}$ states with $\mathrm{n}=4$ through 10 were also seen. A series of resonances below the $n=6$ threshold were observed as dips In the partial cross section into $\mathrm{H}^{\circ}(n=5)$.

PHOTODETACHMENT

H ION, SPECTROSCOPY

BEGATIVE HYDR.OGEN ION

HYDROGEN, ELECTRON AFFINITY 
INTRODUCTION

A. Motivation and Background

The negative hydrogen ion is a stable configuration of one proton and two electrons, formed when a neutral hydrogen atom is polarized by the electric field of a second electron. The electron is bound to the induced dipole with an energy of approximately $0.75 \mathrm{eV}$. This configuration is the ${ }^{1} \mathrm{~S}$ ground state of $\mathrm{H}^{-}$and is the only stable state for the ion. There are also numerous doubly excited states or resonances corresponding with the excited states of $\mathrm{H}^{\circ}$. These unstable states autodetach very rapidly into an ejected electron plus a hydrogen atom which is often in an excited state.

In the experiments to be described photodetachment of one or both elestrons from $\mathrm{H}^{-}$ions is observed and the relative cross section for each process is measured as a function of photon energy from threshold to $15.6 \mathrm{eV}$. The one and two-electron photodetachment processes are renresented symbolically as 


$$
\begin{aligned}
& \mathrm{H}^{-}+\mathrm{hv}+\mathrm{H}^{\circ}+\mathrm{e}^{-} \quad\left(1 \mathrm{e}^{-} \text {photodetachment }\right) \\
& \mathrm{H}^{-}+\mathrm{hv}+\mathrm{H}^{+}+2 \mathrm{e}^{-} \quad\left(2 \mathrm{e}^{-} \text {photodetachment }\right)
\end{aligned}
$$

where $h v$ is the effective photon energy in the ion's rest frame. The experimental technique consists of crossing an intense laser beam with a relativistic $\mathrm{H}^{-}$ion beam and counting the $\mathrm{H}^{\circ}, \mathrm{H}^{+}$and $\mathrm{e}^{-}$particles produced by the photodetachment reactions. Due to the extreme ion velocity the laser photons appear to be highly Doppler shifted. A fixed frequency laser is thus continuously tuned over the region of interest by varying the angle of intersection between the laser and ion beams.

The $\mathrm{H}^{-}$ion is one of the simplest systems to describe quantim mechanically. Due to its simple structure, the $\mathrm{H}^{-}$ ion has been of interest since the advent of quantum mechanics. Bethe (1929) and Hylleraas (1930) both calculated the binding energy and radial distribution of $\mathrm{H}^{-}$.

While the Hamiltonian for this two-electron system is exactly known, the properties are a challenge to calculate. The $\mathrm{H}^{-}$problem is fundamentally different from the related case of helium, in that the outer electron is not bound in a Coulomb field but is attached by core polarization and exchange effects. These weaker interactions which may be treated as perturbations in the case of helium must be accurately accounted for to obtain even 
approximate results for $\mathrm{H}^{-}$. The correlated motion of the electron is essential to the description here. Analytic wave functions for $\mathrm{H}^{-}$are not available but approximate solutions of great accuracy may be obtained using modern numerical computing techniques. The ground state binding energy has been calculated by Pekeris (1962) and Aashamar (1970) to an accuracy of one part in $10^{9}$ using variational techniques.

Since the $\mathrm{H}^{-}$problem is complex but tractable, it has served as a proving ground for the validity of theoretical models and their extension to other more complicated systems. This may prove especially true in the case of two-electron photodetachment near the threshold. In order to predict the correct results for this process the theory must properly describe the dynamics of the correlated motion of the two electrons. The quantum mechanics of three separated charged particles has been called the most important unsolved problem in non-relativistic atomic physics today.

Two-electron photodetachment from $\mathrm{H}^{-}$is closely related to electron impact ionization of an atom. This is a fundamental process in all forms of electric discharge through a gas. Simflarly the doubly excited states in $\mathrm{H}^{-}$ are the same resonance states observed in electron scattering from hydrogen.

The $\mathrm{H}^{-}$ion is of great importance in astrophysics. wildt (1939) first suggested bound free emission of $\mathrm{H}^{-}$as 
a primary mechanism explaining the opacity of the solar atmosphere. In the following decade the $\mathrm{H}^{-}$ion played a central role in tìe theories of stellar atmospheres. $\mathrm{H}^{-}$ is formed by radiative recombination of low energy electrons and hydrogen atoms. Radiation from the surface of the star is absorbed by photodetachment. The cycle of absorption and reradiation occurs over and over again. The net result is a modification of the radiation spectrum. The total energy radiated is unchanged but the emission spectrum is shifted.

The $\mathrm{H}^{-}$ion is of great practical importance. $\mathrm{H}^{-}$ions are accelerated at the Los Alamos Meson Physics Facility (LAMPF) and other high energy accelerators. A better understanding of the ion may lead to more efficient design and use of these accelerators. A most practical application is the generation of high energy neutral beams for heating plasms in magnetically confined fusion reactors. The $\mathrm{H}^{-}$beam can be easily accelerated, then stripped of one electron by photodetachment to produce a ne11tral hesm. Such neutral beams can penetrate the strong confining fields of the magnetic bottle to heat plasma contained inside.

B. Calculations and Measurements of Photodetachment Cross Section.

The process of $\mathrm{H}^{-}$photodetachment has received considerable theoretical attention. Chandrasekhar (1945) 
first calculated the photodetachment cross section. This calculation covered the energy region below the $n=2$ threshold where only the $H(n=1)$ final state is energetically accessible. Numerous others, for instance Geltman (1962) and Ajmera and Chung (1975), have performed calculations considering only the channel into the $n=1$ final state. These calculations give improved accuracy but are in basic agreement with the earlier work of Chandrasekhar. A11 predict the one-electron cross section to rise smoothly from threshold near $0.75 \mathrm{eV}$ to a broad peak of approximately $4 \times 10^{-17} \mathrm{~cm}^{2}$ near $1.5 \mathrm{eV}$ and then fall smooth1y with increasing energy.

Since these calculations ignore channels into excited hydrogenic states, they give incorrect results above the $\mathrm{n}=2$ threshold at $10.95 \mathrm{eV}$. They also cannot be expected to predict the resonance features. Macek (1967) and also Hyman et al. (1972) have performed close coupling calculations above the $\mathrm{n}=2$ threshold. These calculations give branching ratios to $n=1$ and $n=2$ final states. More recently Broad and Reinhardt (1976) have performed a multichannel calculation over the energy range of 0 to $70 \mathrm{eV}$ using the J-matrix formulation of close-coupling theory. They obtain the total cross section as well as partial cross sections for photodetachment channels into $\mathrm{H}$ $(n=1), H(n=2)$ and $H^{+}$. The channel producing $\mathrm{H}^{+}$is the two-electron cross section. 
In figure 1 the total cross section calculated by Broad and Reinhardt is plotted versus photon energy. Also plotted are the partial cross sections into $H(n=1)$ and $H$ $(n=2)$. Notice the form of the channel into $n=2$. There is a very sharp rise from threshold, then a gradual decrease in cross section for increasing energy. It is assumed that similar behavior occurs for $n=3$ and the higher excited states. This behavior is important in the interpretation of the results of the present experiment and is discussed in more detail in following chapters.

Also plotted on the figure are the experimentally measured relative values of Smith and Burch (1959). These are the classic data of the fleld. The data of Smith end Burch are seen in more detail in figure 2 and are listed in Table 1. These relative cross sections cover the energy range of $0.95 \mathrm{eV}$ to $2.9 \mathrm{eV}$ with a resolution of approximately $0,06 \mathrm{eV}$. They were obtained by crossing the photon beam from a filtered carbon arc continuum source with a low velocity $\mathrm{H}^{-}$beam and counting photodetached electrons. Data which extend to lower energy have been obtained by Feldman (1970) using similar techniques with a monochromatized xenon arc source. The relative measurements have been normalized to absolute integrated measurements of Branscomb and Smith (1955).

Structure near $n=2$ is apparent in figure 1 . This structure is seen in more detail in figure 3 which is the 


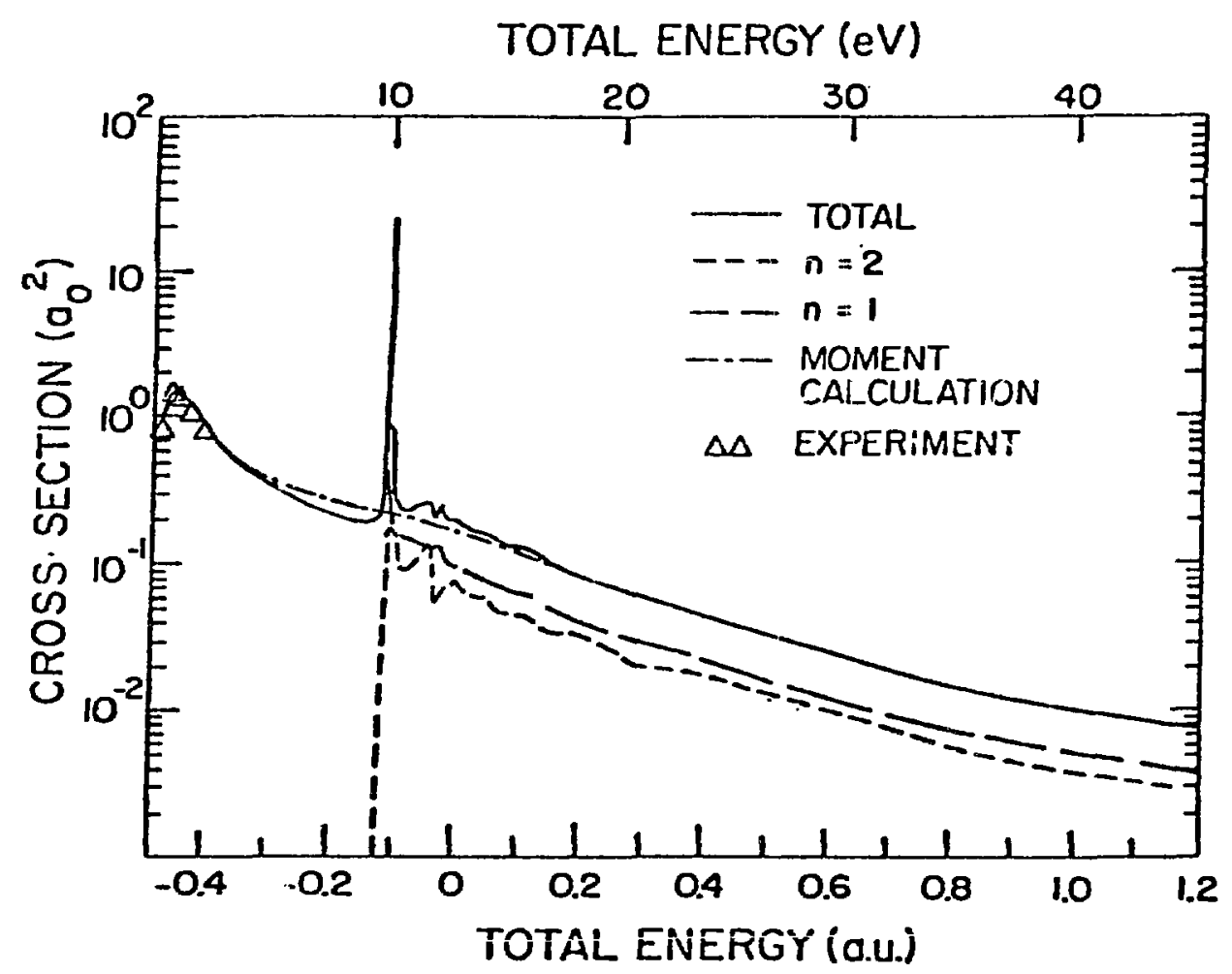

Figure 1. Photodetachment Cross Section of $\mathrm{H}^{-}$. From J. T. Broad and W. P. Reinhardt. 


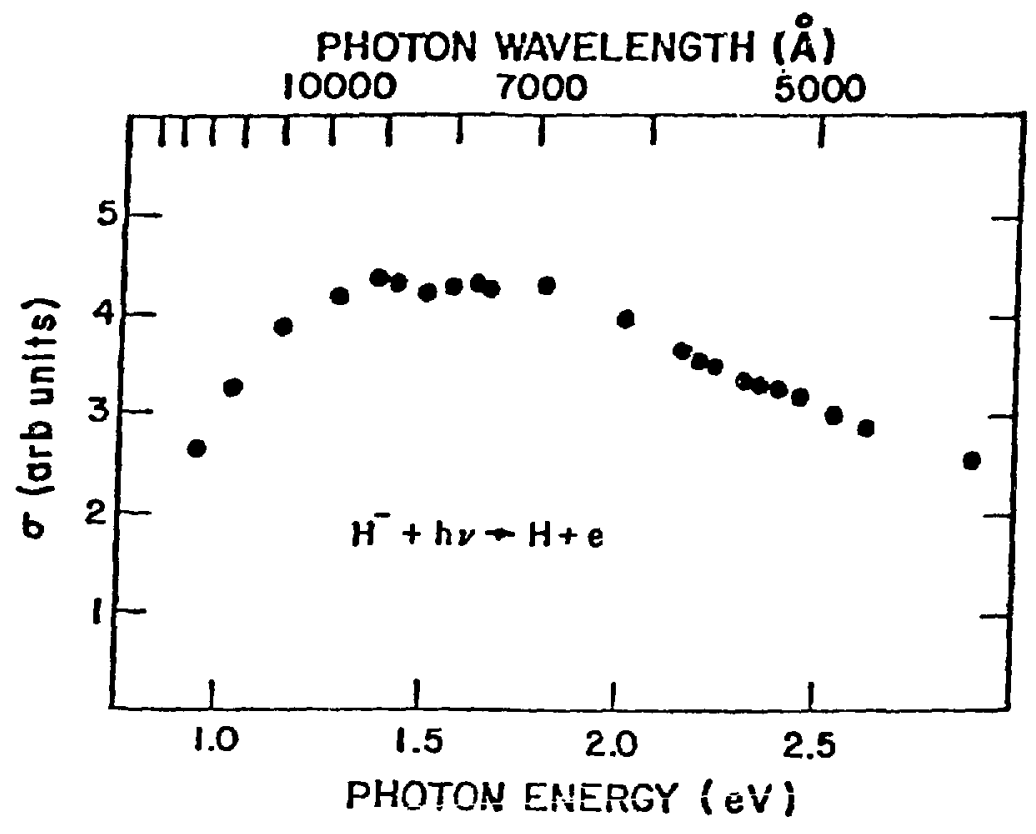

Figure 2. Experimental Photodetachment Relative Cross Section of Smith and Burch. After Smith and Burch. 
Table 1. Relative $\mathrm{H}^{-}$Photodetachment Cross Section, $\frac{\sigma\left(\lambda_{\mathrm{m}}\right)}{\sigma\left(\lambda_{1}\right)}$.

\begin{tabular}{|c|c|c|c|}
\hline Filter & $\begin{array}{c}\text { Effective } \\
\text { Wavelength } \\
\lambda_{\mathrm{m}} \text { (Angstrom) }\end{array}$ & $\sigma\left(\lambda_{\mathrm{m}}\right) / \sigma\left(\lambda_{7}\right)$ & $\begin{array}{l}\text { Probable } \\
\text { Error } \\
(\%)^{r}\end{array}$ \\
\hline 1 & 4260 & 0.76 & 3.0 \\
\hline 3 & 4620 & 0.87 & 2.5 \\
\hline 4 & 4880 & 0.91 & 2.0 \\
\hline 5 & 5060 & 0.95 & 1.5 \\
\hline 6 & 5180 & 0.98 & 1.5 \\
\hline 7 & 5280 & 1.00 & \\
\hline 8 & 5340 & 1.02 & 1.2 \\
\hline 9 & 5560 & 1.05 & 1.2 \\
\hline 10 & 5620 & 1.07 & 1.3 \\
\hline 11 & 5760 & 1.10 & 1.4 \\
\hline 12 & 6170 & 1.19 & 1.2 \\
\hline 13 & 6890 & 1.29 & 1.3 \\
\hline 14 & 7500 & 1.28 & 1.8 \\
\hline 22 & 7520 & 1.29 & 1.7 \\
\hline 23 & 7640 & 1.29 & 2.6 \\
\hline 24 & 7650 & 1.31 & 1.6 \\
\hline $25 x$ & 7940 & 1.29 & 1.9 \\
\hline 25 & 8270 & 1.28 & 1.9 \\
\hline 26 & 8740 & 1.31 & $1: 9$ \\
\hline 28 & 8960 & 1.30 & 1.7 \\
\hline 27 & 9020 & 1.32 & 1.6 \\
\hline 29 & 9700 & 1.26 & 1.7 \\
\hline 32 & 10770 & 1.18 & 2.6 \\
\hline 33 & 12010 & 0.98 & 2.7 \\
\hline 34 & 13010 & 0.79 & 2.7 \\
\hline
\end{tabular}




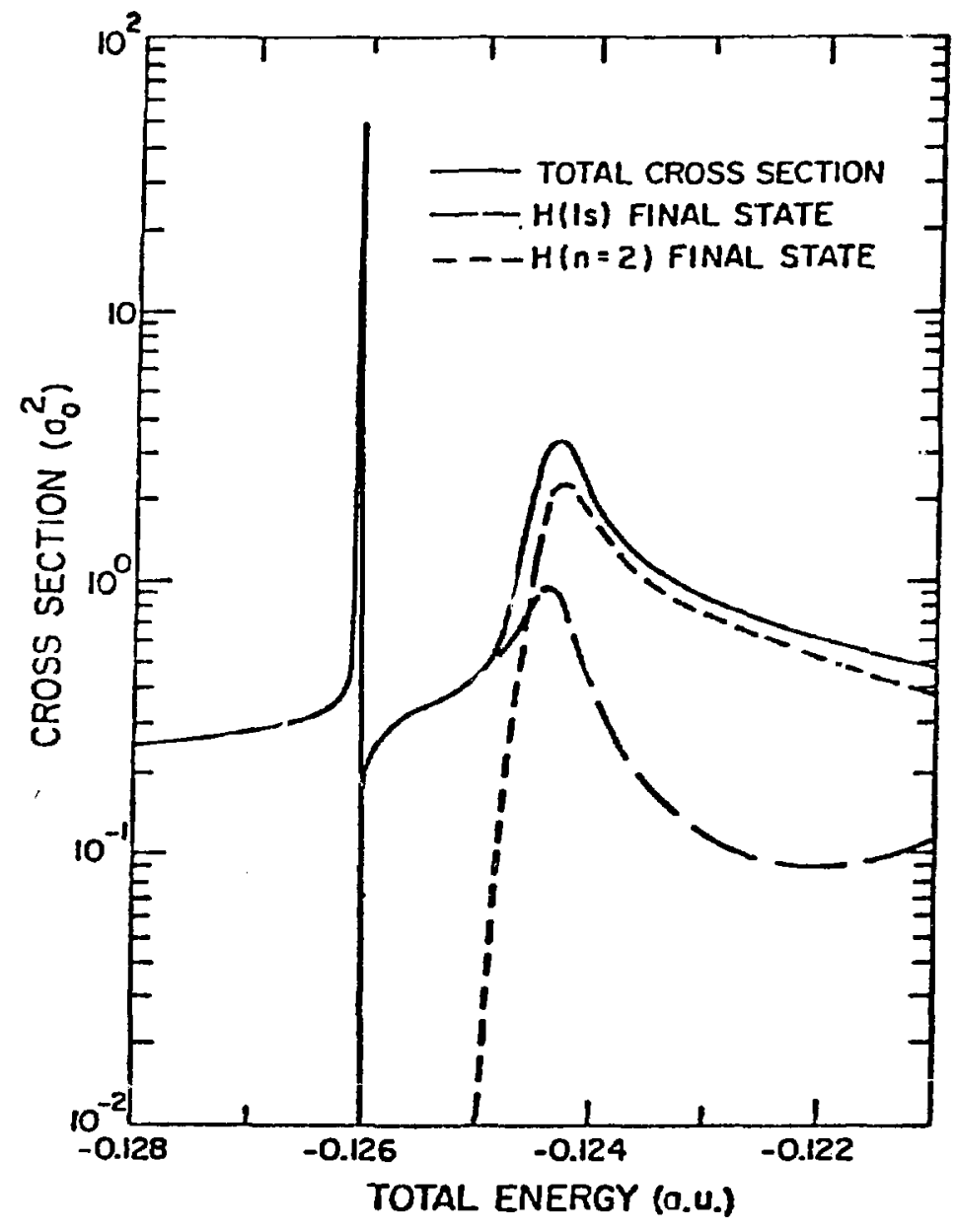

Figure 3. $\mathrm{H}^{-}$Photodetachment Cross Section in the Resonance Region. From J. T. Broad and W. P. Reinhardt. 
result of another calculation by Broad and Reinhardt (1976) using a more diffuse basis set optimized for the $\mathrm{n}=2$ threshold region. The one-electron cross section in square Bohrs $\left(a_{0}^{2}=2.80 \times 10^{-17} \mathrm{~cm}^{2}\right)$ is plotted as a function of the photon energy relative to the two-electron threshold in atomic units (a.u. $=27.2 \mathrm{eV}$ ). The narrow resonance below the threshold is the lowest member of a Feshbach series converging on $n=2$. The broad peak above threshold is the $n=2$ shape resonance. Both of these resonance features have been clearly observed by our group (Byrant et al 1977) and have been thoroughly discussed as the topic of a previous dissertation (Tootoonchi 1977). The Doppler-tuned-colliding beam technique pioneered by these measurements has also been employed (Hamm et a1 1979) to observe resonance features associated with $n=3$ and the continuum from 1.5 to $10.5 \mathrm{eV}$ (Sharifian 1977). Figure 4 is a plot of the two-electron cross section calculated by Broad and Reinhardt as a function of photon energy above the two-electron threshold in atomic units. The peak in photodetachment occurs approximately 0.1 a.u. above the threshold or at a photon energy of approximately $17 \mathrm{eV}$. At peak the two-electron cross section is predicted to be approximately 0.007 square Bohrs. The threshold for two-electron photoejection occurs at 14.35 eV. The authors polnt out that the two-electron calculations are not well converged below $22 \mathrm{eV}$. Thus the 


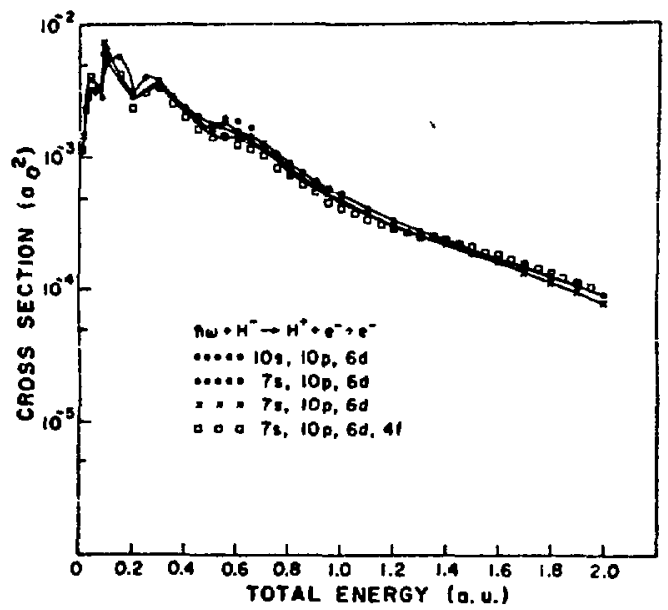

Figure 4. Two-electron Cross Section Calculated by Broad and Reinhardt. 
magnitude, as well as location of the peak, is not well determined by the calculation.

Figure 5 shows the two-electron cross section compared to the one-electron cross section. This $\log -\log$ plot demonstrates the dominance of the one electron process. This figure contains the key features of the calculations of Broad and Reinhardt. These calculations have agreed well with our measurements and provide a framework for the analysis of our results. Table 2 lists the values calculated for the total cross section as a function of wavelength.

The behavior of the photodetachment cross section near threshold is very interesting. Generalizations can be made about the shape of the cross section at threshold without specifying the exact atomic system under study or its particular wave functions. Wigner (1948) considered two charged particles and showed that threshold cross section behavior is determined by the long-range interaction and is independent of short-range forces.

For the case of photodetachment, the dominant long range interaction is the $\frac{\ell(\ell+1)}{r^{2}}$ potential barrier term. In this case the Wigner threshold law is $\sigma \sim \mathrm{E}_{\mathrm{k}}$ $\frac{(2 \ell+1)}{2}$ where $\sigma$ is the cross section, $E_{k}$ is the kinetic energy of the ejected electron and $l$ its orbital angular momentum quantum number. For photodetachment from the $\mathrm{H}^{-}$ground 


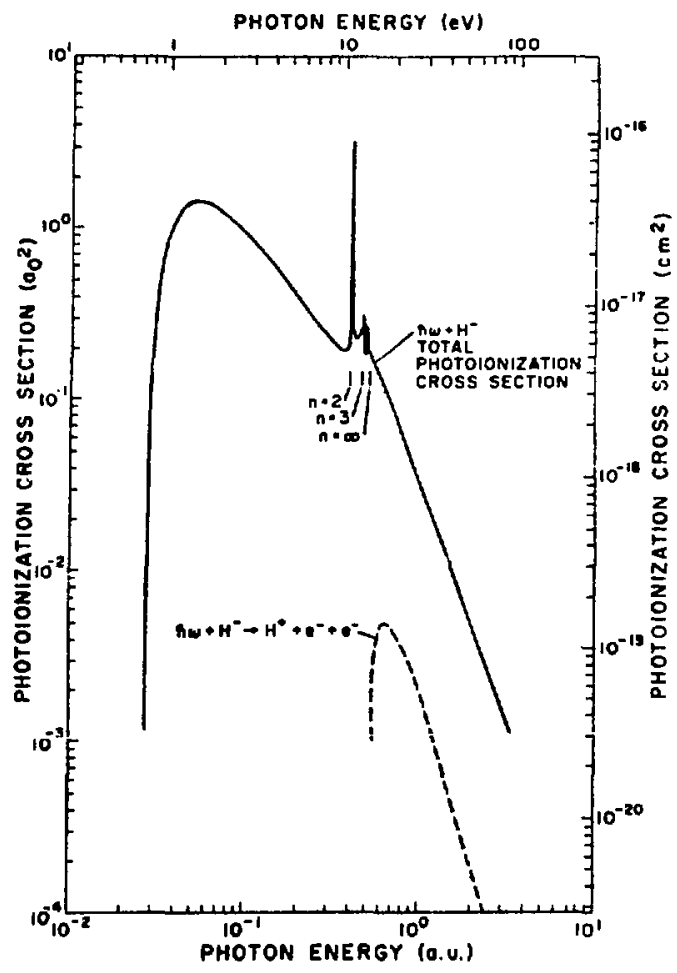

FIgure 5. Comparison of One and Two-Electron Cross Sections as calculated by Broad and Reinhardt. 
Table 2. $\mathrm{H}^{-}$photodetachment cross section calculated by Broad and Reinhardt as a function of wavelength.

\begin{tabular}{|c|c|}
\hline $\begin{array}{c}\text { Wavelength } \\
\text { (nm) } \\
\end{array}$ & $\begin{array}{l}\text { Calculated } \\
\left(10^{-17} \mathrm{~cm}^{2}\right) \\
\end{array}$ \\
\hline 1219.0 & 2.80 \\
\hline 962.0 & 3.98 \\
\hline 794.0 & 3.95 \\
\hline 676.0 & 3.76 \\
\hline 589.0 & 3.31 \\
\hline 528.0 & 3.02 \\
\hline 358.0 & 1.98 \\
\hline 257.0 & 1.31 \\
\hline 200.0 & 0.951 \\
\hline 164.0 & 0.729 \\
\hline 139.0 & 0.591 \\
\hline 121.0 & 0.543 \\
\hline 62.6 & 0.233 \\
\hline 49.1 & 0.124 \\
\hline 40.4 & 0.0703 \\
\hline 34.3 & 0.0414 \\
\hline 29.8 & 0.0283 \\
\hline 26.4 & 0.0206 \\
\hline 23.6 & 0.0155 \\
\hline 21.4 & 0.0177 \\
\hline 19.6 & 0.0088 \\
\hline 18.0 & 0.0067 \\
\hline
\end{tabular}


state to the $H^{\circ}$ ground state, the efected electron must be $P$ wave since both ground states are $S$ states while the photon carries one unit of angular momentum. Then the threshold law goes as $E_{k}^{3 / 2}$. Here $E_{k}=E-E_{0}$ where $E$ is the photon energy and $E_{0}$ the binding energy of $\mathrm{H}^{-}$.

There is no a priori way to assess the energy range over which the law applies. As the energy excess increases, the power will depart from $3 / 2$. This is due in part to the effects of short range interactions between the neutral $\mathrm{H}$ and the departing electron. Analysis of the range of the law and its departure from the limiting threshold behevior gives information about the nature of the short range forces. The calculation of Broad and Reinhardt predicts the $3 / 2$ power threshold law for oneelectron photodetachment.

For the case of photodetachment of ${ }^{1} \mathrm{~S}$ ground state $\mathrm{H}^{-}$into excited $\mathrm{H}^{\circ}$ states the ejected electron is not limited to $\mathrm{P}$ wave. Hyman et al (1972) have shown that the photodetachment channel to $\mathrm{H}^{\mathrm{O}}$ (2P) dominates near the $\mathrm{n}=2$ threshold. In this case the ejected electron is $S$ wave giving a Wigner threshold dependence of $E_{k}^{1 / 2}$. We expect similar behavior for higher excited states.

The Wigner threshold theory does not apply to the case of two-electron photodetachment since this is definitely a three-body problem. One cannot assume the model of 
one electron partially screening the proton from the other. Such a situation would lead to the screening electron being bound with only one electron ejected. In order to achieve double escape at threshold the two electrons must remain at the same distance from the proton but on opposite sides (Fano 1974). The motion is strongly correlated. This strongly correlated motion is not adequately described by the independent particle model.

The only way in which both electrons may be ejected at threshold is to share the photon energy equally. As the photon energy is increased above threshold, there are more ways of distributing the photon energy which will allow double escape. If the energy were shared randomly between the two electrons, the cross section would rise linearly from threshold. This is not expected.

Wannier (1953) has solved the problem of electron impact Ionization classically. This is the problem of three charged bodies. His argument considers the family of allowed escape trajectories as a function of energy. The result of Wannier's analysis is that the cross section varies as $E_{k}^{n}$ where $E_{k}$ is the kinetic energy of the ejected electron and $\mathbf{n}$ is a simple function of the nuclear charge. For the case of $\mathrm{H}^{-}$photodetachment with charge cf one, $\mathrm{n}=$ $1.12689 \ldots$ This is the generally accepted form of the two-electron photodetachment threshold behavior for $\mathrm{H}^{-}$. 
This result is referred to as the "Wannier threshold law." The most recent treatments have tended to confirm the behavior predicted by Wannier. The result of Peterkorp (1970) and Rau (1971) give the same form. There are numerous other results which agree on the form of the law but predict $n$ from 1.0 to 1.5 . These results as well as the derivation of 'Aannier's Law are discussed in more detail in the following chapter.

Temkin (1974) has suggested a modulated linear law which predicts oscillations in cross section near the threshold. The form of the two-electron threshold is currently a subject of controversy.

There have been no reported observations of twoelectron photodetachment from $\mathrm{H}^{-}$previous to the current experiments. There have been experiments with electron impact lorization of helium which seem to suggest that the Wannier law is correct (Marchand et al. 1969) (Cvejanaovic and Read 1974). These experiments cannot select the correct form from the many conpeting theories due to their 1 imited energy resolution. A high resolution measurement of the two-electron photodetachment threshold behavior is required to settle the controversy.

$C_{\text {n }}$ Experimental Method

In order to study the threshold laws, one needs a source of narrow linewidth radiation. This is essential 
for the study of a threshold law since the law may be valid for only a few meV above the threshold. The crossed beam technique as employed by Smith and Burch and also Feldman would be ideal if the filtered arc lamp were replaced with a tunable laser. This would give the intensity and resolution required for the study of a threshold law. To study the single-electron threshold near $0.75 \mathrm{eV}$, a tunable dye laser could be employed (Lineberger 1980).

Unfortunately lasers producing wavelengths short enough to study two-electron threshold at $14.353 \mathrm{eV}$ do not exist. It is highly unlikely that tunable lasers for this range will be developed in the near future.

The problem of obtaining a short wavelength tunable source was solved by Bryant et al (1977) with the crossed beam Doppler shifted laser technique used to observe the $\mathrm{n}=2$ Feshbach and shape resonances. In the current experiment this technique is extended to the measurement of the photodetachment cross section near the one and twoelectron thresholds. In this technique the beam from a standard laboratory laser is directed to intersect a high velocity $\mathrm{H}^{-}$ion beam. Due to the high velocity, laser photons may be Doppler shifted through a large energy range by varying the angle of intersection between the " beams. The electrons produced by photodetachment reactions are magnetically deflected out of the beam and into a particle counter. 
In order to use thĭs 'trick', one needs a source of the target ions at very high velocity. The linear accelerator at the Los Alamos Meson Physics Facility (LAMPF) provides an $\mathrm{H}^{-}$beam with a velocity which is $84 \%$ the speed of light. The relativistic Doppler equation gives the photon energy $\mathrm{E}$ in the frame of the $\mathrm{H}^{-}$ion (which is also the C.M. frame) in terms of the laboratory energy of the laser $E_{L}$ and the angle of intersection of the beams. $\alpha$ is defined as zero for a head-on collision. The Doppler equation is

$$
E=\gamma(1+\beta \cos \alpha) E_{L}
$$

where $\beta$ is the ratio of ion velocity to the speed of light and $\gamma=\left(1-\beta^{2}\right)^{-1 / 2}$. For the $800 \mathrm{MeV} \mathrm{H}$ beam at LAMPF $\beta=0.842$ and $\gamma=1.85$. For this case the term $\gamma(1+\beta$ $\cos \alpha$ ) ranges from 0.293 to 3.41 . This provides a very large energy tuning range. A larger range is obtained by using harmonics of a Nd:YAG laser. Figure 6 shows the energies available with several common lasers as a function of the intersection angle. Using the first four harmonics of the YAG laser gives an energy range from $0.34 \mathrm{eV}$ to $15.9 \mathrm{eV}$. Thus the spectrum from near IR to VUV is covered.

The rate of photodetachment reactions (photodetachments/second) is proportional to the product of the cross 


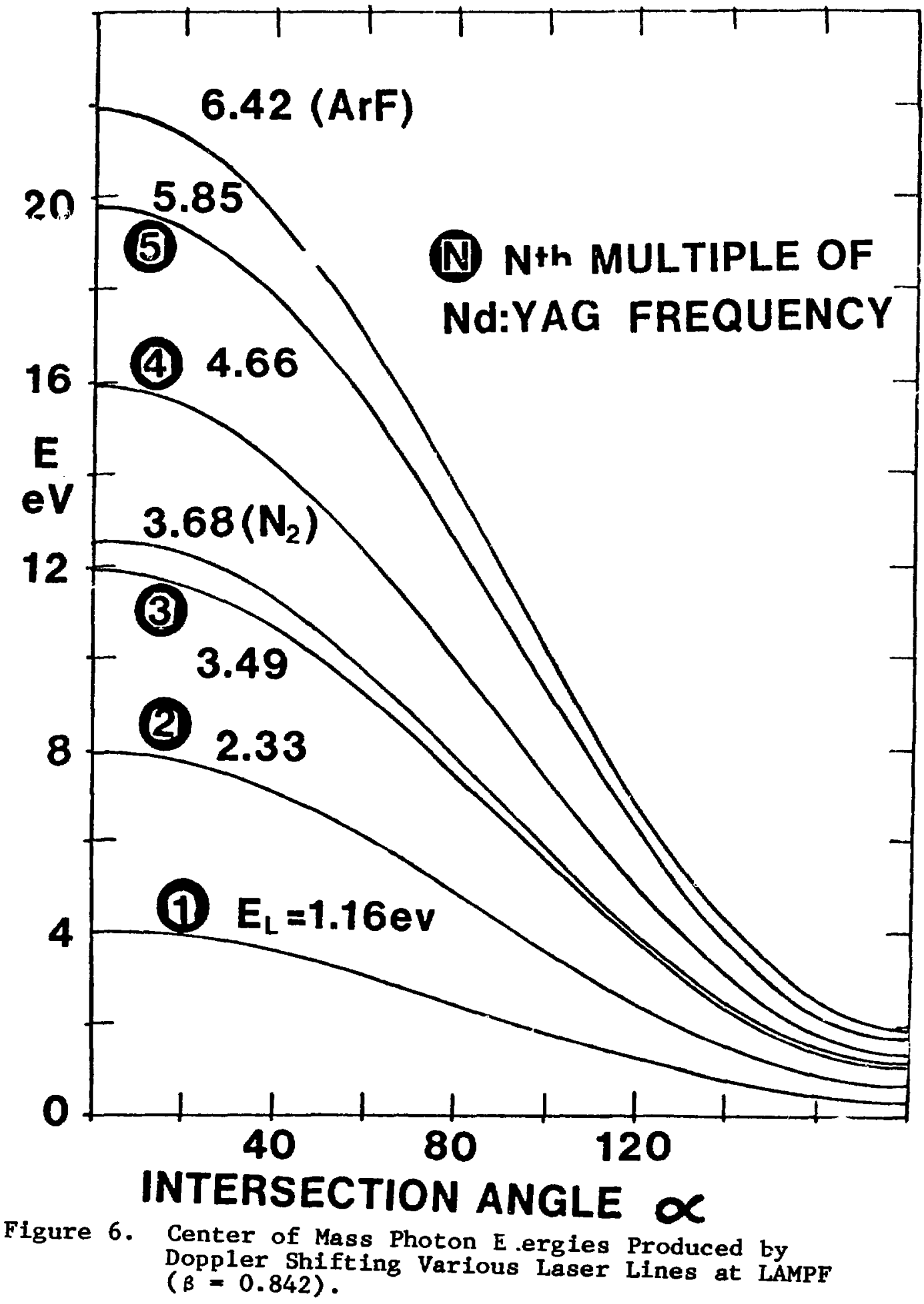


section for the process, the lon beam current, and the photon beam current. The relativistic rate equation is given by Bryant et al (1971) as

$$
R=\sigma \operatorname{IJG}\left(\frac{1+\beta \cos \alpha}{\beta \sin \alpha}\right)
$$

where $R$ is the detachment rate in counts per second, $\sigma$ is the photodetachment cross section and $\alpha$ is the intersection angle. I and $J$ are the photon and ion currents respectively in particles per second. G is a factor which depends on th $a$ overlap of the cwo beams in time as well as space. For two continuous, perfectly overlapping cylindrical beams of radius $r$,

$$
G=\frac{16}{3 \pi^{2} r c}
$$

where $c$ is the speed of 1ight. Derivation of the relativistic rate equation is given by Sharifian (1977).

The photodetached particles $\left(\mathrm{H}^{\circ}, \mathrm{H}^{+}, \mathrm{e}^{-}\right)$are directed into particle counters by a deflecting magnet. The $\mathrm{H}^{\circ}$ signal results from one-electron photodetachments while the $\mathrm{H}^{+}$signal is due to two-electron photodetachments. Both processes contribute to the $\mathrm{e}^{-}$signal. The cross section is calculated from the measured rate and other known quantities using equations 4 and 5 .

Another relativistic effect which one must consider is the transformation of the magnetic deflecting field. 
The transverse magnetic field generates a perpendicular electric field which is given by the Lorentz equation $E=\gamma \beta B C$. Due to the extreme velocity of the lons, the modest magnetic deflecting field may be transformed into a huge electric field. For the $800 \mathrm{MeV}$ beam, a magnetic field of 1000 Gauss is transformed into an electric field $468 \mathrm{kV} / \mathrm{cm}$. This huge field can strip electrons from the particles, confusing the results of the experiment. For this reason the magnitude of the magnetic deflecting field is minimized. 
II

THEORETICAL BACKGROUND

\section{A. Photodetachment}

The problem of photodetachment of $\mathrm{H}^{-}$can be formulated within the framework of time dependent perturbation theory. The initial state is the ion in a radiation field. The final state for the one-electron process is a neutral hydrogen atom and a free electron. In the case of two-electron photodetachment, we have a proton with two free electrons for the final state.

In the electric dipole approximation the one-electron problem is that of the dipole moment $\mathrm{p}$ of the $\mathrm{H}^{-}$ion interacting with an oscillating electric field of infinite extent. The probability of a transition per unit time is given by time-dependent perturbation theory as

$$
P \sim \nu\left|\left\langle\Psi_{b}|p| \Psi_{f}\right\rangle\right|^{2} \rho\left(E_{k}\right)=\nu D^{2} \rho\left(E_{k}\right)
$$

where $\rho\left(E_{k}\right)$ is the density of states in the continuum which is proportional to the square root of the energy $E_{k}$ of the ejected electron, and $E_{k}=h v-E_{o}$ where $h v$ is the photon energy and $E_{0}$ the binding energy. $D_{z}$ is the 
electric dipole matrix element between the inftial bound state $\Psi_{b}$ and the final continuum state $\Psi_{f}$.

The photodetachment cross section $\sigma$ will be given by

$$
\sigma \sim \mathrm{UD}_{\mathrm{z}}^{2} \mathrm{E}_{\mathrm{k}}^{1 / 2}
$$

where the density of states has been made explicit.

The dipole matrix element $D_{z}$ may be expressed in the length, velocity, or acceleration forms which are

$$
\begin{aligned}
D_{z} & =\int \Psi_{b}\left(z_{1}+z_{2}\right) \Psi_{f} d \tau & \text { (length) } \\
& =\frac{1}{h \nu} \int{ }_{b}\left(\frac{\partial}{\partial z_{1}}+\frac{\partial}{\partial z_{2}}\right) \Psi_{f} d \tau & \text { (velocity) } \\
& =\frac{1}{h v} 2 \int \Psi_{b}\left(\frac{z_{1}}{r_{1}^{3}}+\frac{z_{2}}{r_{2}^{3}}\right) \Psi_{f} d \tau & \text { (acceleration) }
\end{aligned}
$$

where $z_{i}$ is the component of the radius vector $r_{i}$ of the ith electron along the oscillating field (Chandrasekhar 1945) .

If the wave functions $\psi_{b}$ and $\psi_{f}$ were exact solutions, all three forms would be equivalent. Since only approximate wave functions are avallable, the three forms give different results. The degree of convergence between the three results is a test of the 'quality' of a given pair of wave functions.

The dipole operator in the matrix element may be considered as a weighting on the wave function which emphasizes different regions of the coordinate space depending 
on the form chosen. Chandrasekhar (1945) first noted that the most accurete cross section is computiad from the velocity form since the accuracy of the wave functions is highest for intermediate distances from the: proton. These regions are emphasized by the velocity form. The length form emphasizes regions of large $r$, while the acceleration form weights the interior region most highly. A large number of calculations for the photodetachment cross section into the $\mathrm{H}^{\mathrm{O}}$ ground state have been performed using this method. These calculations have demonstrated increasing accuracy over the years as more complete wave functions were employed.

The earliest calculations (for example Chandrasekhar 1945) employed for the final state wave Finction the product of an unperturbed hydrogen atom ground state with a plane wave for the ejected electron. More recent calculations (Chandrasekhar and Elbert 1958) have used a distorted wave for the ejected electron with the same unperturbed $\mathrm{H}^{\circ}$ state. These continuum states have been used with increasingly accurate Hylleraas-type $\mathrm{H}^{-}$bound state functions determined by the Ritz variational principle of energy minimization. Such calculations (Chandrasekhar 1958, Chandrasekhar and Elbert 1958, John 1960) are virtually identical in shape and differ by only a few percent in magnitude for the velocity form of the 
matrix element. They give considerable disagreement between results obtained with length, velocity, and acceleration forms (Bates 1962).

More recently Geltman (1962) has used a 70-parameter bound-state function and a many-term correlated free-state function to obtain a cross section that gives essential agreement with the data of Smith and Burch (1959) within their experimental error. This function gives much better convergence of length, velocity, and acceleration forms than previous calculations.

A very simple parameterization for the $\mathrm{H}^{-}$cross section has been obtained by Armstrong (1963). This simple analytic expression is obtained by using a $V=0$ solution for the bound state for all $r$ rather than only for large $r$. This gives a bound state wave function of the form

$$
\psi_{b} \sim \frac{1}{r} \exp \left[\frac{\sqrt{\frac{m E_{o}}{h}}}{r}\right]
$$

This is used with a plane wave for the electron to obtain

$$
\sigma(h v) \sim E_{O}^{1 / 2} \frac{\left(h v-E_{o}\right)^{3 / 2}}{(h v)^{3}}
$$

This simple form demonstrates the key features of the oneelectron process. These are

a) Threshold at $E_{0}$

b) Limiting threshold behavior of $\sigma \sim\left(h \nu-E_{O}\right)^{3 / 2}$

c) Broad maximum in cross section near $2 \mathrm{E}_{\mathrm{o}}$ 
Armstrong introduces an empirical correction factor which is a function of photon energy to obtain better agreement with the data of Smith and Burch (1959). The corrected formula is

$$
\sigma(h v) \sim \frac{E_{O}^{1 / 2}\left(h \nu-E_{0}\right)^{3 / 2}}{(h v)^{3}}\left[1+\frac{g_{0}}{2} E_{o}\left(2 E_{O}-h v\right)\right]^{2}
$$

where $E_{0}=0.745 \mathrm{eV}$ and $g_{0}=4.7$ (when energies are expressed in Rydbergs) gave best fit to the data of Smith and Burch.

The previous calculations consider only the channel into the $\mathrm{H}^{0}$ ground state. The recent calculation of Broad and Reinhardt (1976) which is not limited to this channel gives close agreement to the calculation of Geltman (1962) and other single channel calculations for the region below $\mathrm{n}=2$ where they are equivalent. Above the $\mathrm{n}=2$ threshold, the results of the multichannel calculation give substantial differences as one would expect. These results were described in chapter one.

All of the theories have been tested by comparison to the measurement of Smith and Burch over a limited energy region. It is clear that another experimental measurement of the one-electron photodetachment cross section would be extremely valuable. This would be particularly true for a measurement which covered the entire energy range from below threshold $(.75 \mathrm{eV})$ to above the 
series limit $(14.35 \mathrm{eV})$. For this reason we have measured the one-electron cross section over this extended range.

\section{B. One-Electron Threshold}

Certain generalizations can be made regarding the threshold behavior of a system. These are based on its overall symmetry properties rather than on the detalls of the specific syster under study. Wigner (1948) in a classic paper showed that the limiting energy dependence of a cross section at threshold is the same function of the excess energy no matter what the reaction mechanisms so long as the long range interaction between the particles is the same. He considered only the two-body problem for the three cases of Coulomb attraction, Coulomb repulsion, and no long range force. Wigner divided the problem into an inner region or reaction zone and an outer region where only the long range forces apply. He showed that knowledge of the complex process in the reaction zone is not required to determine the threshold behavior. He obtained threshold laws from the asymptotic forms of solutions to the two-body Schrödinger equation in the outer zone.

Wigner obtained the threshold law for each possible case of reaction or scattering process of two particles. The relevant case for the one-electron photodetachment reaction is the creation of two new particles with angular 
momentum $l$ when there is no Coulomb interaction. This is appropriate to the ejection of an electron from the neutral core. Here the dominant pseudopotential in the equivalent ore dimensional problem at large distances is the $\frac{\ell(\ell+1)}{r^{2}}$ angular momencum term. For this case Wigner obtains

$$
\sigma \sim \mathrm{k}^{2 \ell+1}
$$

where $k$ is the momentum or wave number of the ejected electron.

The range of validity of the Wigner law cannot generally be determined a priori. Wigner points out three effects which limit the range of validity of the asymptotic behavior. These are as follows:

a) The region must be small compared with the distance of resonances from each other.

b) We must have ka $<<1$ where $k$ is the wave number of the ejected electron and $a$ is the radius of the reaction zone.

c) The range of validity can be reduced by an add:tional potential term.

Branscomb (1958) considered the case of photodetachment of negative ions in more detail. He showed that the threshold behavior is given by 


$$
\sigma(v) \sim v^{2 \ell+1}\left(a_{0}+a_{1} k^{2}+a_{2} k^{4}+\ldots\right)
$$

where $v$ is the photon frequency, $k$ is the momentum of the ejected electron and $\ell$ is the lowest angular momentum component of the continuum state to which an electric dipole transition is allowed from the bound state.

Since the ejected electrons are nonrelativistic we have $E_{k} \sim k^{2}$ where $E_{k}$ is the energy of the ejected electron and for the one-electron threshold of $\mathrm{H}^{-}$we have

$$
\sigma \sim v E_{k}^{3 / 2}\left(a_{o}+a_{1} E_{k}+a_{2} E_{k}^{2}+\ldots\right) \text {. }
$$

For $\mathrm{P}$ electron shells such as $\mathrm{C}^{-}, \mathrm{O}^{-}$, etc.,

$$
\sigma \sim v E_{k}^{1 / 2}\left(a_{0}+a_{1} E_{k}+a_{2} E_{k}^{2}+\cdots\right) \text {. }
$$

This result should also apply to the $\mathrm{H}^{-}$thresholds for photodetachment into excited $\mathrm{H}^{\circ}$ states.

The effect of short range forces on the threshold laws is poorly understood. It has been suggested that the inclusion of a polarization potential $\left(-a r^{-4}\right)$ may cause the appearance of logarithmic terms and odd as well as even powers of $k$ in the expansion of equation 15 .

The range of validity of the threshold law must be determined by measurement. In the case of $\mathrm{S}^{-}$photodetachmenc (Lineberger 1970) to the neutral ground state the simple $3 / 2$ power law is valid to within a few percent for 
a range of approximately $20 \mathrm{meV}$ above threshold. Measurements on photodetachment of $\mathrm{Cs}^{-}$to the first ${ }^{2} \mathrm{P}$ state (Slater 1976) indicate a range of $0.1 \mathrm{meV}$ is appropriate. Extreme experimental resolution is required to verify the asymptotic behavior in this case. It is believed that all one-electron photodetachment processes follow the Wigner law when very close to the threshold.

C. Two-Electron Threshold

Wigner's threshold theory is limited to the case of two final particles and does not directly apply to twoelectron photoejection which is a three-budy problem. In theory the threshold cross section for photoejection can be calculated as

$$
\sigma_{t h}(E) \sim \rho(E) \mid\left\langle\Psi_{b}|p| \Psi_{f}>\left.\right|^{2}\right.
$$

where $E$ is the photon energy excess above threshold. The phase space term $\rho(E)$ is multiplied by the appropriate matrix element. The density of states $\rho(E)$ which was proportional to $\mathrm{E}^{1 / 2}$ for the one-electron process will be proportional to $\mathrm{E}^{2}$ for the two-electron photodetachment (Rau 1971).

This method worked quite we11 for the one-electron case, as was demonstrated by close agreement with the $3 / 2$ power law at threshold for all the calculations including the simple model of Armstrong. 
In the case of two-electron photodetachment exactly the same Initial bound state wave function is involved, but for the final state the full two-electron continuum wave function must be employed. The correct functions are the quantum solutions of the three-body problem. These were discussed as early as 1937 (Bartlett 1937), but even today the two-electron continuum functions are poorly understood.

Bates et al (1950) employed perhaps the simplest continuum functions. He represented both ejected electrons as Coulomb waves and thus obtained a threshold cross section which was linear in energy. His representation is equivalent to saying that neither electron screens the other from the central field. This ignores the electronelectron interaction. Such behavior would be the limiting case of two electrons ejected from a highly ionized core of large number charge $z$.

Another approach assumes one electron totally shields the other from the field of the nucleus. The final state is then the product of a Coulomb wave for the electron which provides the shielding with a plane wave for the outer electron. This assumption results in a threshold cross section which varies as the three halves power of the excess energy above threshold. This behavior is equivalent to the case of single-electron ejection and cannot lead to two-electron escape. 
Neither of these results can be ccrrect since they Ignore the mutual screening between the two-electrons which is determined by the partition of the available energy between them. The energy dependent screening is determined by the correlated motion of the two electrons. These correlation effects become increasingly important as the energy of the electrons approaches zero. Fano (1974) has fointed out the importance of correlated motion in providing high $\ell$ states near the two-electron threshold. These states should exist both above and below the threshold even when the overall state possesses zero angular momentum. That is, the continuum (or Rydberg) wave functions include higher spherical harmonics of either electron even when the total wave function is restricted to $\mathbf{S}$ state.

Complete shielding of one electron by the other gives $\sigma \sim E^{3 / 2}$ while no screening gives $\sigma \sim E$. Due to the energy dependent mutual shielding or 'Dynamic Screening' (Rau 1971) we expected $\sigma \sim E^{n}$ where $n$ is between $3 / 2$ and one.

Due to the seemingly intractable problem of obtaining the true two-electron continuum wave functions, Wannier (1953) approached the problem of electron impact ionization of atoms classically. He applied classical mechanics to obtain trajectories outside the reaction zone and 
assumed that trajectories from the inner zone fil1 the available phase space. This gave $\sigma \sim E^{\mathrm{n}}$ where $\dot{n=1.127}$.

Wannier's work was the first to consider the mutual screening of the two-electrons and forms the basis of our present understanding of the two-electron ejection process. Because of its importance I will trace a few of the key steps.

If an electron is incident on an atoin, the $S$ wave coupling will dominate and the final state will have zero angular momentum. This simplifies the Hamiltonian which may be witten in terms of 3 variables $r_{1}, r_{2}$, and $\theta_{12}$ and their conjugate momenta. Here $r_{1}$ and $r_{2}$ are the distances of the electrons from the ion and ${ }^{\theta_{1}} 12$ is the angle between them.

A change of variable gives

$$
\begin{aligned}
& r_{1}=R \cos (1 / 2 x) \\
& r_{2}=R \sin (1 / 2 x) \\
& R=\left(r_{1}^{2}+r_{2}^{2}\right)^{1 / 2} \\
& 0 \leq x \leq \pi
\end{aligned}
$$

Symmetry in the two electrons is reflected as symmetry in $x$ about $\pi / 2$.

The space is naturally divided into 3 zones in terms of hyperradius $R$. These are 1 ) an inner reaction zone of 
atomic dimensions, 2) a Coulomb zone where the potential dominates, and 3) a free zone. As in Wigner's 1948 development, knowledge of the process in the reaction zone is not required to determine the threshold behavior. Motion in the two outer zones is shown to be classical.

The Hamiltonian is analyzed in the outer zones to obtain families of trajectories which lead to single and double electron escape. For the case of zero energy excess only orbits with $x=\pi / 2$ and $\theta_{12}=\pi$ lead to double escape. All other orbits degenerate into $x=0$ or $x=\pi$ and thus lead only to single escape.

As the energy excess becomes non-zero other orbits open up which allow double escape. It is shown that the relative number of orbits allowing double escape from the reaction zone is a function of energy and varies as $E^{1 / 2 \mu-1 / 4}$ where

$$
\mu=1 / 2[(100 z-9) /(4 z-1)]^{1 / 2}
$$

Here $\mathrm{Z}$ is the nuclear charge of the ion.

Wannier points out that his derivation is not rigorous because of its reliance on ergodicity. The rate of ionization is derived by computing the volume in phase space which escapes per second from the reaction zone and is also subtended by those orbits allowing two electrons to escape. Wannier states "This volume will shrink as the 
energy excess above threshold is reduced; this shrinkage I presume to be the variation of cross section with energy". The density of points of the system in phase space is assumed to be constant in the neighborhood of the reaction zone. This is the ergodic assumption.

If this is true then the cross section is proportional to the relative number of orbits allowing double escape and we have

$$
\sigma \sim E^{1 / 2 \mu-1 / 4}
$$

where $\mu$ is given by equation 20 .

For the case of electron impact ionization of an atom $\mathrm{Z}=1$ and this gives $\sigma \sim \mathrm{E}^{1.127 \ldots}$. Note that the Wannier exponent approaches one as $\mathrm{Z}$ approaches infinity. This is equivalent to the case of no screening.

This result is referred to as the Wannier law. The derivation also predicts the probability distribution $P_{\theta}\left(\theta_{12}\right)$ of angles between the two outgoing electrons has a maxinum at $\theta_{12}=180^{\circ}$. Wannier's classical argument was later extended by Vinkalins and Gailitis (1967) who showed that the width of $\mathrm{P}_{\theta}\left(\theta_{12}\right) \sim \mathrm{E}^{1 / 2}$.

Peterkorp and Liepirsh (1969) have used the WKB approximation in one dimension to confirm Wannier's result. Peterkorp (1970) has extended the WKB treatment to 3 dimensions and obtained solutions which correspond 
with the orbits given by Wannier and also confirmed o $\sim E^{1.127 \ldots .}$. Rau (1971) has obtained two electron continuum solutions to the Schrodinger equation by a similar approximation method. He obtained exact agreement with Wannier's result from one set of solutions.

All of these derivations parallel Wannier's original work very closely and apply most of the same assumptions including ergodicity. They also expand about the Wannier direction $\left(r_{1}=r_{2}, \theta_{12}=\pi\right)$ and can thus be expected to arrive at the same threshold law. There have also been other calculations which predict $\sigma \sim$ E (Rudge and Seaton 1965) or $\sigma \sim E^{\mathrm{n}}$ where $\mathrm{n}$ is between 1 and 1.5 (Temkin 1966, Temkin 1968, Kang 1970). Temkin's approach is based on continuation of the higher excited states into the continuum.

Most recently Temkin and Hahn (1974) have predicted a modulated linear law. They consider the problem of $S$ wave scattering directly using an optical-potential approach and making the simplification $\frac{1}{r_{12}}=\frac{1}{r_{1}+r_{2}}$ in the electronelectron repulsion term of the Hamiltonian. This method does not obtain a unique threshold law but does predict oscillation (of unknown frequency) in cross section near threshold. They suggest a threshold law of the form

$$
\sigma \sim E[1-C \sin (A \ln (E)+B)]
$$


where $A, B$, and $C$ are constants with the only restriction being $|C| \leq \frac{1}{\sqrt{2}}$. This form gives a monatonically increasing function of $E$ with oscillations increasing in frequency as threshold is approached. With the proper choice of constants this form can give results which are quite similar to the Wannier law in the experimentally accessible region.

It should be pointed out that all the treatments of the two-electron problem have considered on $1 \mathrm{y}$ the $\mathrm{L}=0$ case which is approprlate to $S$ wave coupling in electron impact ionization. The case of two-electron photodetachment has an overall $P$ state for the two-electron continuum wave function since the incident photon must deliver one unit of angular momentum to the ${ }^{1} \mathrm{~S} \mathrm{H}^{-}$ground state.

Rau (1971) stated that the process of electron impact Ionization and two-electron photodetachment were equivalent and would have the same threshold law because "The threshold behavior is a feature of the escape process and does not involve detalls of what goes on in the reaction zone". This argument seems tenuous at best since the angular momentum of the final state is different for the two processes. Temkin (1974) also states without justification that the two processes are equivalent and suggests the laser photodetachment technique as a means of observing his predicted oscillations in cross section. 
We note that equivalency of the two processes may be justified on the grounds that each electron has very high $\ell$ near threshold so that the addition of one more unit of angular momentum to either electron would seem to be of minor consequence. 
III

EXPERIMENT

A. Experimental Setup

As discussed in chapter one the photodetachment cross section is related to the observables by equations 4 and 5. These may be solved for the cross section a giving

$$
\sigma=\text { constant } \times N \times \frac{\beta \sin \alpha}{1 J(1+\beta \cos \alpha)}
$$

Where $\mathrm{N}$ is the number of particles photodetached, I and $J$ the number of photons and lons respectively crossing the interaction region during the sampling period. Here the photodetachment rate and currents are replaced by the total number of particles detached and incident since a pulsed laser is used.

The Doppler shifted photon energy in the $\mathrm{H}^{-}$Ions rest frame is given by equation 3

$$
E=E_{0} Y(1+B \cos \alpha)
$$

where $E_{0}$ is the laboratory energy of the laser which is accurately known. B and $\gamma$ are the relativistic constants 
which are functions of $\mathrm{H}^{-}$beam energy. For the $800 \mathrm{MeV}$ $\mathrm{H}^{-}$Ion beam at LAMPF $\beta=0.842$ and $\gamma=1.85$.

The experimental technique consists of measuring the quantities $N, I$ and $J$ for each angle $a$. $\alpha$ is varied to cover the desired energy range. With the present experiment measuring only relative cross sections, the constant of proportionality need not be considered and the quantities $I$ and $J$ need only be determined to arbitrary scale. The actual number of photodetachment reactions counted, $N$ however, must be correct to allow calculation of uncertainty in the result due to counting statistics.

Figure 7 shows the apparatus schematically. The relativistic $\mathrm{H}^{-}$ion beam is crossed with the photon beam from a Q-switched laser. The intersection angle $\alpha$ is varied by a stepping motor and is accurately measured with a precision shaft angle encoder.

A sweep magnet upstream of the interaction point removes electrons stripped from the $\mathrm{H}^{-}$ions by collisions with gas molecules during their traversal of the long beam transport system. Photodetachment fragments are separated according to species $\left(\mathrm{e}^{-}, \mathrm{H}^{\circ}, \mathrm{H}^{+}\right)$by the sweeping magnets. The relativistic particles are detected as counts in plastic scintillators in tine-colncidence with the laser flash. A count in the $\mathrm{H}^{+}$(proton) detector is the signature of two-electron photodetachment while a count in the 

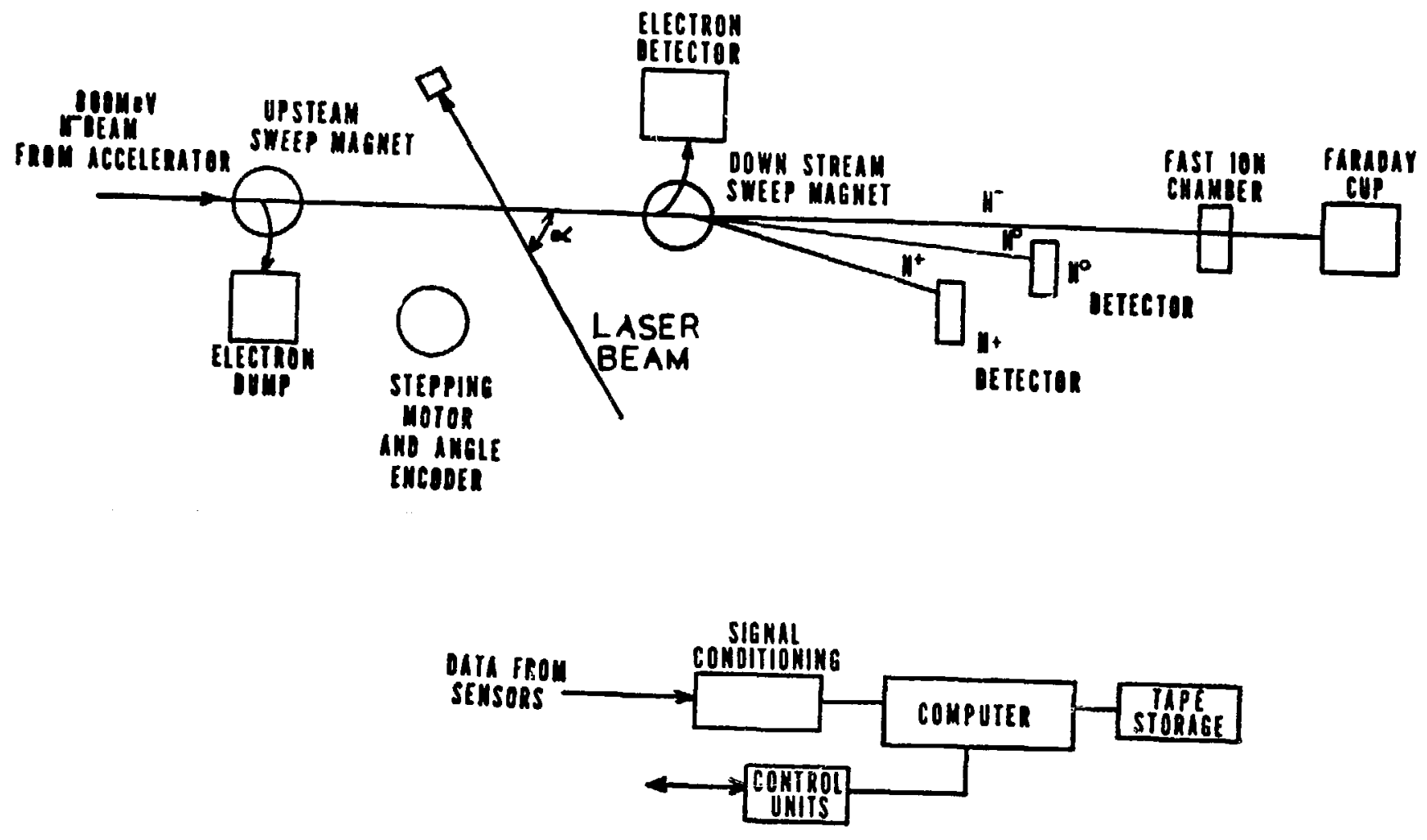

Figure 7. Schematic of Apparatus 
$\mathrm{H}^{\circ}$ (neutral hydrogen atom) detector results from singleelectron photodetachment. The electron detector is sensitive to both processes.

Normalization to $\mathrm{H}^{-}$flux and photon flux is obtained from beam current monitors and photodiodes respectively. The laser provides a train of narrow photon bursts at a constant repetition frequency of 10 pulses per second. After each burst the computer reads and stores digital words which correspond to the quantities $N, J$, and $I$. When sufficient courts are collected to provide preset counting statistics, the sums are recorded giving $\mathrm{N}, \mathrm{J}$, and $I$ for one value of $\alpha$ and its corresponding energy. The angle $\alpha$ is automatically advanced and the process repeats for a new angle. Each angle might require 600 laser shots or 1 minute (at $10 \mathrm{~Hz}$ ) giving a running tine of 1 hour for a typical scan of 60 angles.

The resulting measurements may be displayed graphically in real time. For example, one could display cross section as a function of angle number. At the end of the run a line printer output is generated giving the calculated energy and cross section for each angle as well as other calculated quantities and scaler sums. The sums are recorded on magnetic tape for future analysis.

\section{B. Physical Arrangement}

Figure 8 shows the physical layout of the experiment in the external proton beam (EPB) in area $B$ at LAMPF. The 


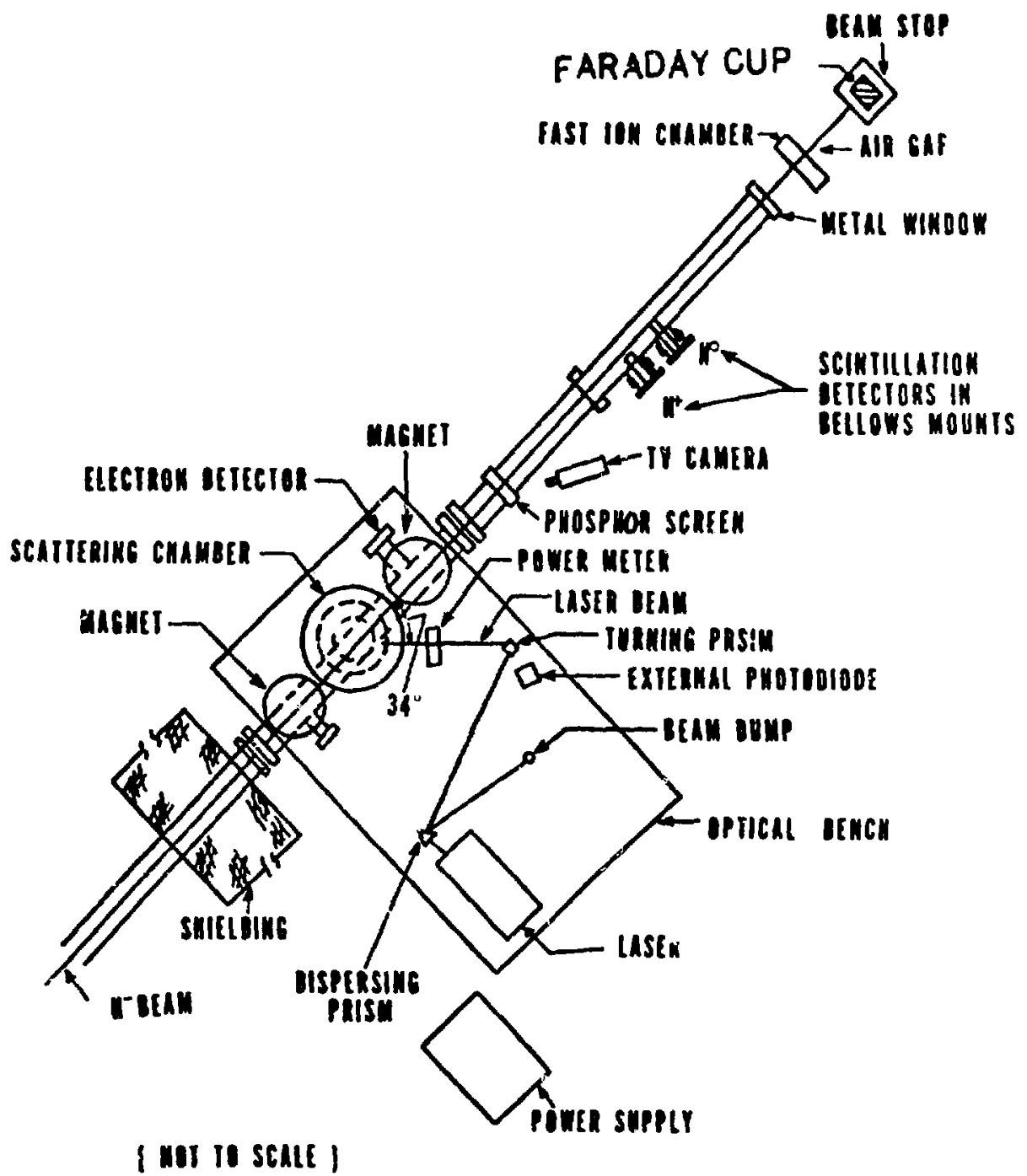

Figure 8. Physical Arrangement 
$800 \mathrm{MeV}$ relativistic $\mathrm{H}^{-}$ion beam enters the scattering chamber where it interacts with photons from the laser. The $\mathrm{H}^{-}$beam is supplied to the photodetachment apparatus through a 6 inch diameter evacuated pipe (beam pipe). Figure 9 is a photograph of the apparatus showing the beam pipe. All major parts of the apparatus were fabricated from type 304 stainless steel to minimize cutgassing. High vacuum is maintained throughout the entire length of approximately one half mile from the $\mathrm{H}^{-}$ion source through the accelerator to the thin metal window at the far end of our apparatus. This 8 inch diameter window is made of $15 \mathrm{mil}$ heat-treated aluminum foil.

Rupture of the window or any other sudden loss of vacuum integrity within our apparatus could launch a shock wave down the beam pipe causing massive damage to the accelerator. For this reason special precautions are required. Standard glass vacuum fittings are not allowed. Instead all vacuum fittings employ stainless steel flange couplings with compression o-rings of aluminum wire or viton.

The scattering chamber must be maintained under extremely high vacuum to minimize gas stripping of the $\mathrm{H}^{-}$beam between the two sweeping magnets which would increase the background count rate in the experiment. To 


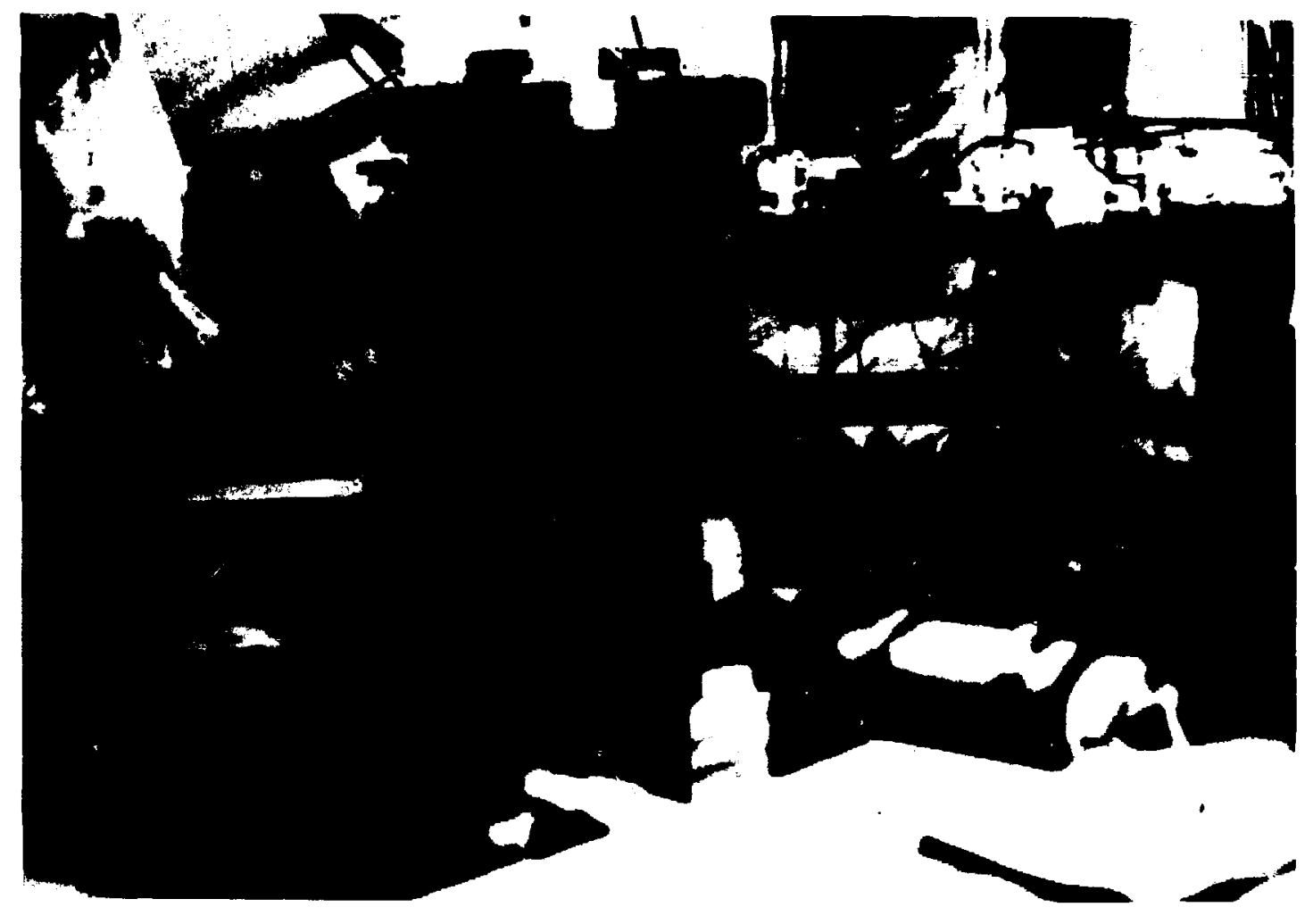

Figure 9. Photograph of Apparatus Showing Beam Pipe. 
this end five massive $600 \mathrm{l} / \mathrm{sec}$ ion pumps are employed. One is coupled to the side of the chamber and the others are attached to the beam pipe near the chamber.

With the above procedures, pressures as low as $8 \mathrm{x}$ $10^{-9}$ torr have been routinely obtained. These pressures are measured inside the chamber with an ionization gauge. The current drawn by the lon pumps and gas stripping rates are also a measure of the pressure.

Due to the extreme angular sensitivity of the measurement, slight motions resulting from flexing of the chamber walls could cause errors in the measurement. The massive scattering chamber designed by David Clark and fabricated in University of New Mexico Physics shop is constructed for maximum rigidity.

Figure 10 is a section view of the chamber showing the mechanism which directs the laser beam across the ion beam. The chamber consists of a 16-inch diameter, thickwalled stainless steel tube mounted with its axis vertically. Four large ports are cut symmetrically in the sides of the tube. Flanges on these ports allow connection of incident and exit beam pipes, ion pump, and pyrex viewing window. Other smaller ports allow penetration for electrical cables, TV camera viewing of mechanism, pressure gauge, etc. The top contains a large removable blank-off flange allowing access to the mechanism inside. The chamber is solidly bolted to the heavy optical table 


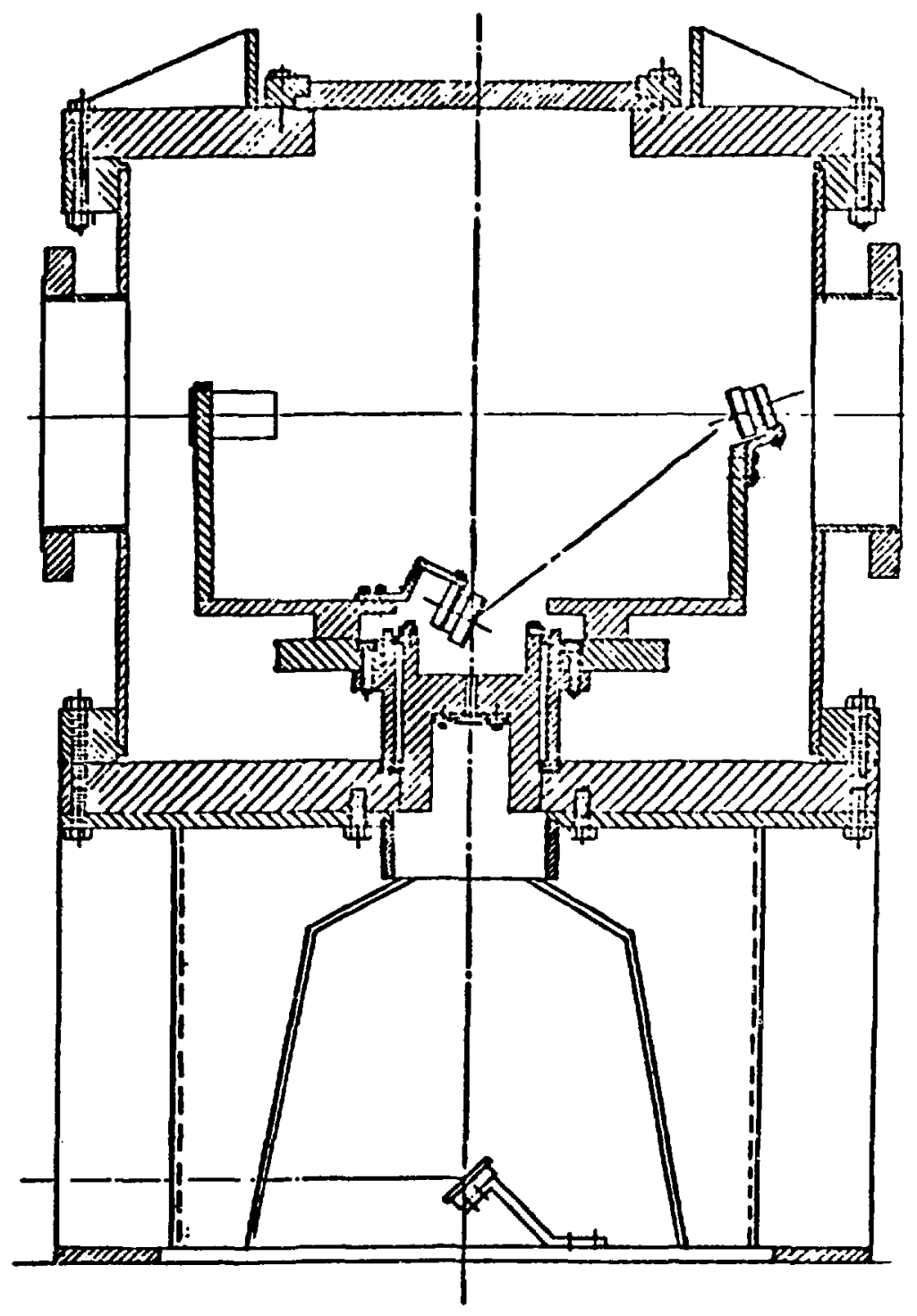

Figure 10. Section View of Chamber Showing Rotating Mirror Arrangement (Spider). 
which supports 1t. Figure 11 shows the robust construction of the chamber.

The laser beam enters the chamber vertically through a quartz window (Suprasil fused silica) which is mounted In a well cut in the bottom plate. The laser beam is then directed horizontally across the $\mathrm{H}^{-}$beam by a pair of mirrors mounted on an arm (referred to as the "spider" for historical reasons). This arm rotates about a vertical axis .

Figure 12 shows the laser beam steering and driving mechanisms schematically. The laser beam which enters the chamber vertically along the axis of rotation of the spider is deflected to cross the ion beam at a variable angle. The spider rotates with the turntable on which it is mounted (See Figure 10). After crossing the $\mathrm{H}^{-}$beam the laser beam is dissipated in a photon dump or catcher mounted on the other end of the spider. The catcher is machined from aluminum in the form of a cone which funnels the photon beam into a cavity where it is absorbed. A solid state photodiode inside the cavity monitors a sample of the photon flux for normalization.

The turntable is driven by a stepping motor through a precision stainless steel belt mechanism. This motor (HS 25 Slosyn, 200 steps/Rev) and 100:1 antibacklash gearbox (DQ - 11 PIC) are mounted to the bottom plate of the chamber. The shaft is coupled through the bottom plate 


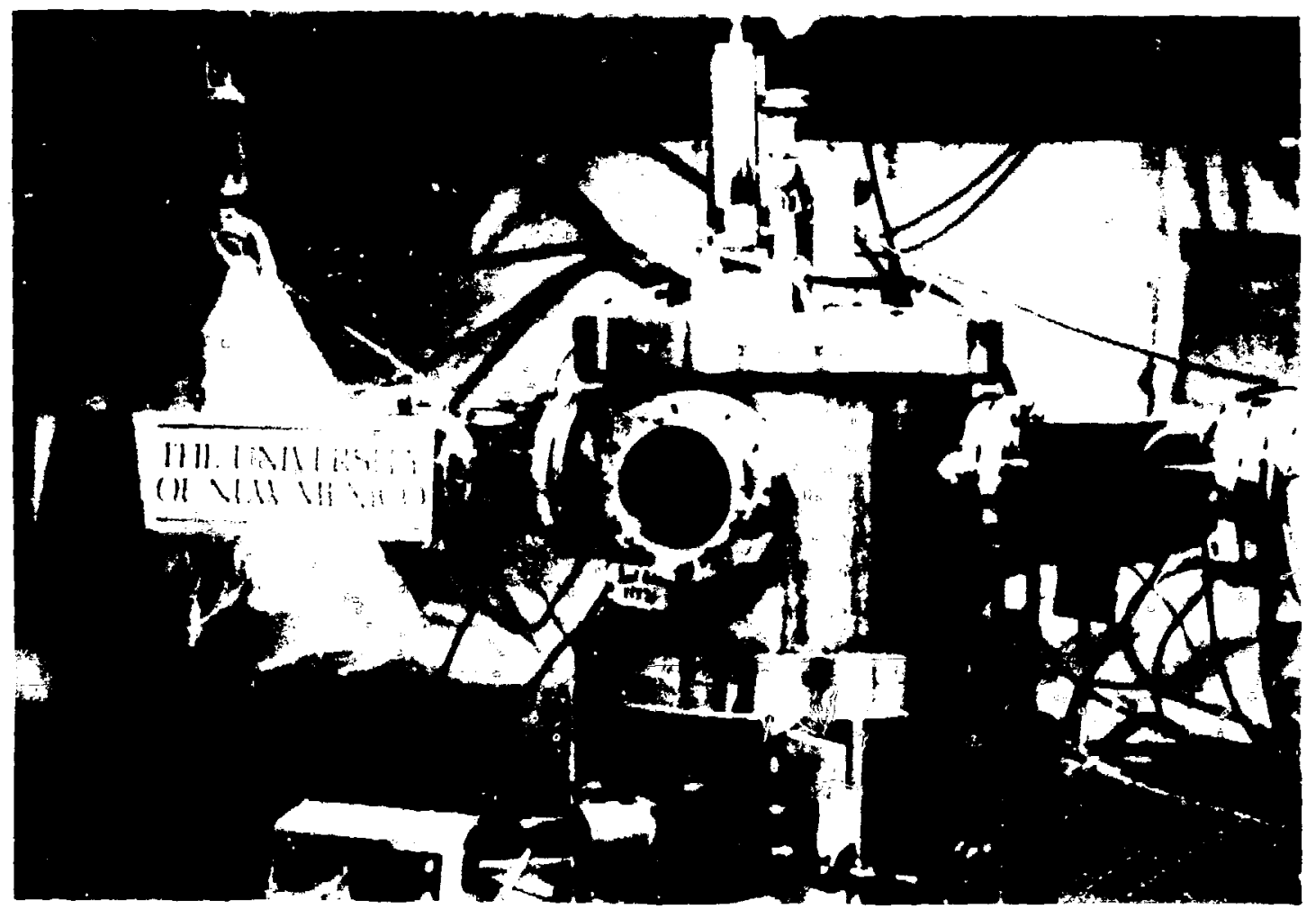

FIgure 11. Photograph of Scattering Chamber 


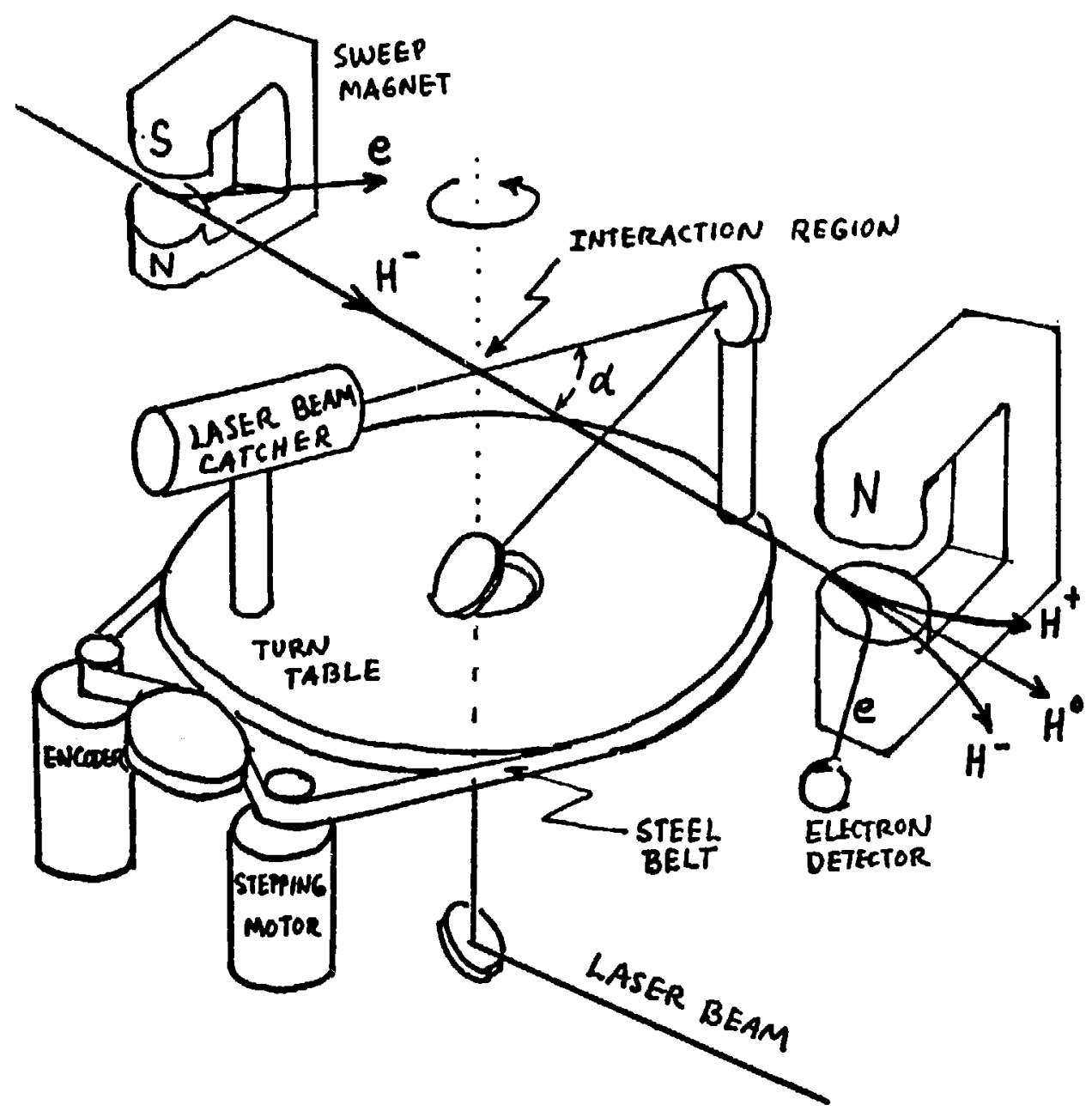

Figure 12. Schematic View of Rotating Mirror Arrangement (Spider) 
with a vacuum tight magnetic feedthrough (SB - 250 rotary feedthrough, Ferrofluidics Corporation) to the belt pulley. The overall mechanical division ratio gives 1 degree of rotation of the spider for approximately 550 notor steps.

The spider angle is measured by a second pulley which is isolated from the driving mechanism to reduce backlash error. Through another magnetic feedthrough, the pulley drives a fourteen bit shaft angle encoder (model 5V42BX, Baldwin Electronics, Inc.) which telemeters the spider angle to the data acquisition system. The angle is actually measured with a resolution of 18 bits. The 360 degrees of possible rotation are divided into 12 sectors. The sector is manually set in binary by four switches. Accuracy and repeatability of the encoding system was checked using a surveyor's theodolite. The calibration was found to be 552.71 encoder steps per degree of rotation. The least significant bit (1 step) corresponds to approximately 31 microradians of angle. The relationship between angle and encoder reading was found to be linear with a small sinusoidal modulation due to bearing wobble. The RMS value of the modulation was less than 3 encoder steps.

The $2 \mathrm{~mm}$ diameter $\mathrm{H}^{-}$ion beam is steered to intersect the center of the $6 \mathrm{~mm}$ diameter laser beam by LAMPF 
operating personnel from the central control room (CCR) using a retractable phosphor screen which is located just down stream of the chamber. This screen is visible by c'osed circuit television from CCR as well as from our experimental trailer. The $\mathrm{H}^{-}$beam is steered vertically to produce maximum photodetachment count rate. This procedure is referred to as the "vertical scan". The beam may be reset with an accuracy of approximately $1 \mathrm{~mm}$. The steering magnet is located approximately four meters upstream from the chamber giving beam angle uncertainty of approximately $0.3 \mathrm{mrad}$ on resetting. The chamber phosphor as well as all upstream phosphors are retracted when not in use to prevent stripping of the $\mathrm{H}^{-}$beam.

C. $\mathrm{H}^{-}$Beam Characteristics

As users of the Clinton P. Anderson Meson Physics Facility (LAMPF), we are provided with a nominal $800 \mathrm{MeV}$ $\mathrm{H}^{-}$ion beam. We have no direct control of the beam. LAMPF operating personnel steer the beam onto our target, adjusting focus and beam current to our specifications. Fluctuations in beam energy, current, and position are inherent in the accelerator and are a constant source of frustration. Indeed they are the primary limitation on accuracy and resolution.

$\mathrm{H}^{-}$ions as well as protons are produced in duoplasmatron ion sources and injected at $750 \mathrm{keV}$ into the 
linear accelerator where they are boosted to an energy of $800 \mathrm{MeV}$. The multi-section accelerator is tuned to maximize current and stability of the primary $\mathrm{H}^{+}$beam. This often results in less than optimum stability in the $\mathrm{H}^{-}$ion beam. Typically the average beam current for the $\mathrm{H}^{+}$beam is $500 \mu \mathrm{A}$ compared with $5 \mu \mathrm{A}$ for the $\mathrm{H}^{-}$beam.

Due to the small beam current requirement of our experiment ( $<100 \mathrm{nA}$ ), the $\mathrm{H}^{-}$beam can be supercollimated. The initial $\mathrm{H}^{-}$ion beam has a diameter in excess of $1 \mathrm{~cm}$. The final adjustable jaw stripper has an opening of typically less than $1 \mathrm{~mm}$ which depends on the ratio of our current requirement to the total $\mathrm{H}^{-}$current. The small point source of the stripper opening is imaged to a nearly parallel beam through our apparatus by a pair of magnetic quadrupole lenses. This arrangement provides an ion beam in our chamber which is typically $2 \mathrm{~mm}$ in diameter with an anguiar divergence of less than $0.2 \mathrm{mrad}$. Separate adjustment of vertical and horizontal quadrupole lenses can provide optimum beam spot for any particular experiment.

While super-collimation provides improvement in angular and spatial characteristics of the $\mathrm{H}^{-}$bean, it does not reduce energy spread of the beam. The beam consists of a group of $\mathrm{H}^{-}$ions with a nominal $800 \mathrm{MeV}$ energy centroid and a more or less Gaussian spread of typically 
$800 \mathrm{keV}$ full width at half aximum (FWHM). The spread has been as large as $1.4 \mathrm{MeV}$ and as small as $50 \mathrm{keV}$ while the centroid has been seen to shift several MeV within one hour. These variations are due to changes in the accelerator. Large bean energy shifts are associated with removal of loading due to the $\mathrm{H}^{+}$beam. For this reason the experiment is automatically gated off whenever the $\mathrm{H}^{+}$beam is absent. These variations in beam energy require constant calibration of the energy scale of the apparatus against a known feature in the $\mathrm{H}^{-}$photodetachment spectrum. The Feshbach resonance (Bryant et al. 1977) serves this purpose well.

The beam current at LAMPF is not steady but rather possesses a complex time structure. Figure 13 compares the beam current tine structure to the duration of the laser flash. The bean tine structure consists of a series of macropulses of 600 us duration with a spacing of 8.33 Ms giving a repetition frequency of $120 \mathrm{~Hz}$. The macropul ses consist of strings of 0.25 ns bursts of current called "micropulses" separated with 5 ns spacing. The laser is tined to fire near the center of the macropulse but coincidence with a micropulse is left to chance. This interplay is considered in the analysis and is seen to affect counting statistics. 


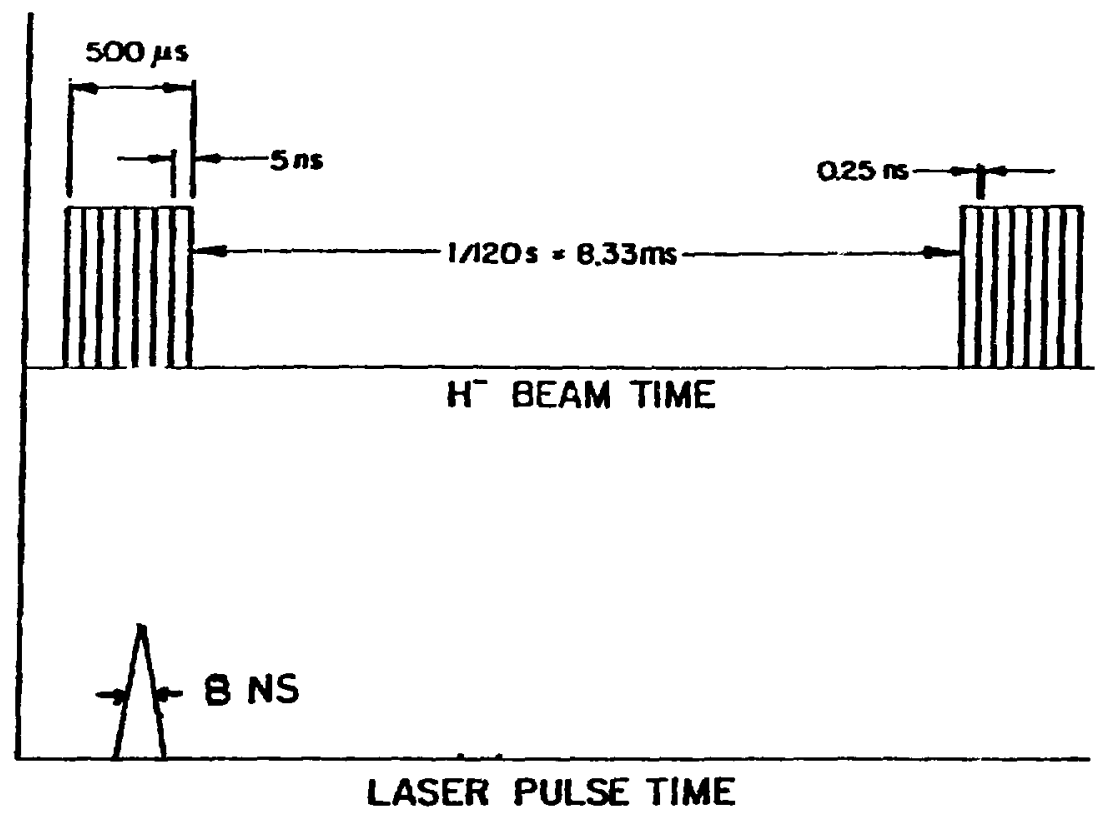

Figure 13. The $\mathrm{H}^{-}$and Laser Beam Structure. 


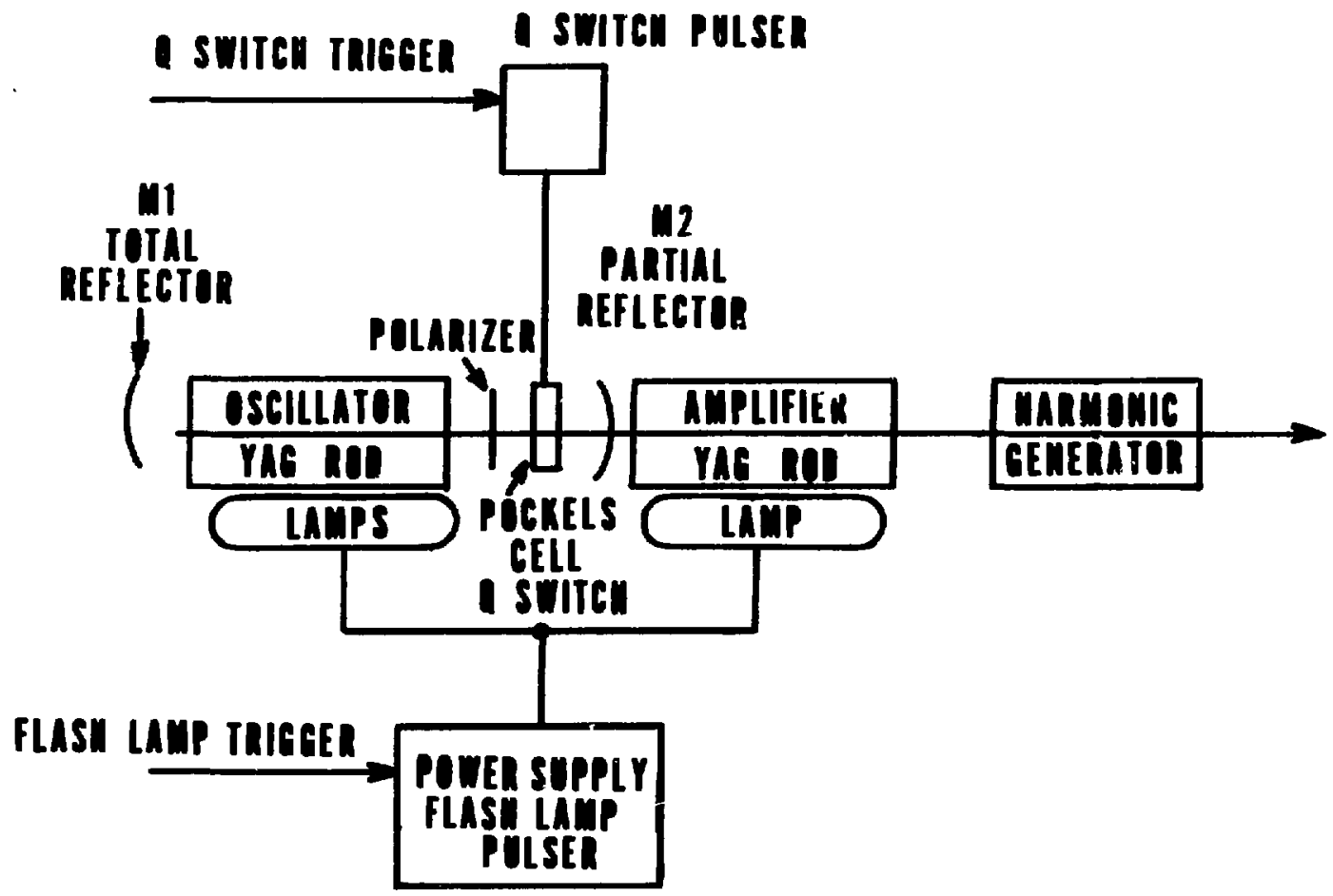

Figure 14. Laser Block Diagram 
optical shutter formed by the combination of the Pockels cell with a crossed polarizer. Opening the shutter restores the $Q$ of the cavity resonator allowing amplification of random noise from rod fluorescence to build to peak oscillation in approximately 5 nanoseconds. Most of the energy stored in the rod is extracted in a laser pulse of approximately 9 nanoseconds duration. The wavelength corresponds with the Nd:YAG fluorescence peak at 1.064 micron.

The burst of photons passes through the amplifier rod, dumping energy stored in population inversion and producing an output pulse of approximately 0.7 joule at a wavelength of 1.064 micron with a temporal width of approximately $8 \mathrm{~ns}$ (FWHM) and an angular divergence of $0.5 \mathrm{mrad}$. Peak power is $8 \times 10^{7}$ watts in a beam $6 \mathrm{~mm}$ in diameter.

The extremely high power densities aillow efficient harmonic generation through non-linear optics. The harmonic generator consists of a pair of room temperature angle-tuned crystals of ammonium di-hydrogen phosphate (ADP). The first stage produces second harmonic energy and also transmits some of the fundamental wavelength. One of two crystals may be used for the second stage. To obtain either the fourth harmonic by doubling of the second or the third harmonic by mixing of fundamental with 
second harmonic. The crystals have faces cut at Brewster's angle. No index matching fluid or antireflective coatings are employed as the extreme power density would cause damage. The crystals are angle-tuned for maximum output by a pair of selsyn motors.

A fifth harmonic generator employing mixing of first and fourth was purchased and briefly tested. This unit manufactured of ADP material by Inrad Corporation provided an output of 4 millijoule per pulse at $212 \mathrm{~nm}$. The unit was unsatisfactory due to rapid accumulation of surface damage. Future experiments with the fifth harmonic appear to be reasonable. Table 3 gives a brief summary of the characteristics of the Quanta-ray DCR-1 laser.

On careful inspection using an ultra fast planar vacuum photodiode, the laser output pulse was found to be, not a simple Gaussian of width $8 \mathrm{~ns}$, but rather, a series of extremely narrow spikes contained within the Gaussian envelope. Interaction of these narrow spikes with the current microstructure of the $\mathrm{H}^{-}$beam pulse had a catastropic effect on the measurements. When $\mathrm{H}^{-}$ion micropulses overlap laser micropulses saturation occurs. The $\mathrm{H}^{-}$beam is totally stripped by the extreme peak laser power level. When $\mathrm{H}^{-}$micropulses fall to overlap laser micropulses no photodetachment occurs. This effect caused a non-linear response and produced non-Poisson counting statistics. 
Table 3. Specifications for Quanta-Ray DCR I Laser.

DCR Laser System output characteristics at $10 \mathrm{~Hz}$ repetition rate

\begin{tabular}{cccc} 
Wavelength(nm) & $\begin{array}{l}\text { Pulse } \\
\text { Width(ns) }\end{array}$ & $\mathrm{mJ} /$ pulse & $\begin{array}{l}\text { Peak } \\
\text { Power(MW) }\end{array}$ \\
\hline 1064 & $8-9$ & 700 & 80 \\
532 & $6-7$ & 225 & 32 \\
355 & $5-6$ & 125 & 20 \\
266 & $4-5$ & 60 & 12 \\
\hline
\end{tabular}

Pulse repetition rate: User Selectable, 2 to 22 pps. Average power constant above 15 pps.

Beam diameter: $\quad 6.5 \mathrm{~mm}$, nominal.

Beam divergence: Near diffraction limit, $<0.5 \mathrm{mr}$. full angle.

Output pulse fitter: <5ns from Q-switch sync. pulse output (typically 3ns).

Linewidth $1064 \mathrm{~nm}$ :

$<1 \mathrm{~cm}^{-1}$

with Intra-Cavity

$$
\text { Etalon }
$$

$<0.2 \mathrm{~cm}^{-1}$

with Electronic

Line Narrowing

$<0.02 \mathrm{~cm}^{-1}$

kated flashlamp life: $3 \times 10^{7}$ shots 
A search of the literature revealed an explanation of the problem as mode beating or mode spiking. The random phase combination of several cavity resonator modes falling within the fluorescence amplification band of the laser medium produces a series of narrow spikes of periodicity $2 \mathrm{~L} / \mathrm{c}$, where $\mathrm{L}$ is the cavity length and $c$ the speed of light.

This is similar to the effect which is employed in mode locked lasers to achieve extremely narrow pulses. Computer simulation of the process confirmed the fact that the observed waveform could be the result of mode spiking. To simulate the process the Gaussian envelope was multiplied by the sum of sinusoids of random phase and a frequency spacing $220 \mathrm{MHz}$ (the mode spacing resulting from physical dimensions of the oscillator cavity). The sinusoids were weighted with a Gaussian distribution of amplitude coefficients centered at the fluorescence peak. The results of several simulations showed waveforms similar to those observed experimentally. Some spikes thus produced had widths of less than 0.1 ns. One expects this is the typical output time structure for pulsed lasers of this type.

The solution to the mode spiking problem was an accessary from the laser manufacturer. The spiking produces a line-broadening of the laser line explained by 
Fourier transformation of the temporal structure into the frequency domain. An accessory available from Quanta-Ray Inc. called "Electronic I.ine Narrowing (ELN)" narrows the output to a single mode or at most two or three modes. An ELN unit was purchased and eliminated the mode the spiking problem. Observations with the fast vacuum photodiode confirmed the near Gaussian time structure. After installation of the ELN, Poisson counting statistics were obtained.

Operation of the ELN is as follows. A photodiode is coupled to the oscillator cavity via a fiber optics light guide. This photodiode monitors the spontaneous noise emission within the cavity. When optimum flashlamp pumping is achieved, a large noise spike is sampled. On the following noise spike the Q-switch is fired by a fast thyratron circuit. This technique produces single mode laser output in accord with the natural oscillation phase of the system.

Figures 8 and 15 show the elements of the optical train. The fundamental laser wavelength together with harnonics are enitted colinearly through the laser output aperture. These various conponents are separated by a Pellin-Broce dispersing prism. The unwanted harmonics are dumped in photon bean dumps ade from copper tubing. The desired harmonic is directed to a 90 degree turning prism 


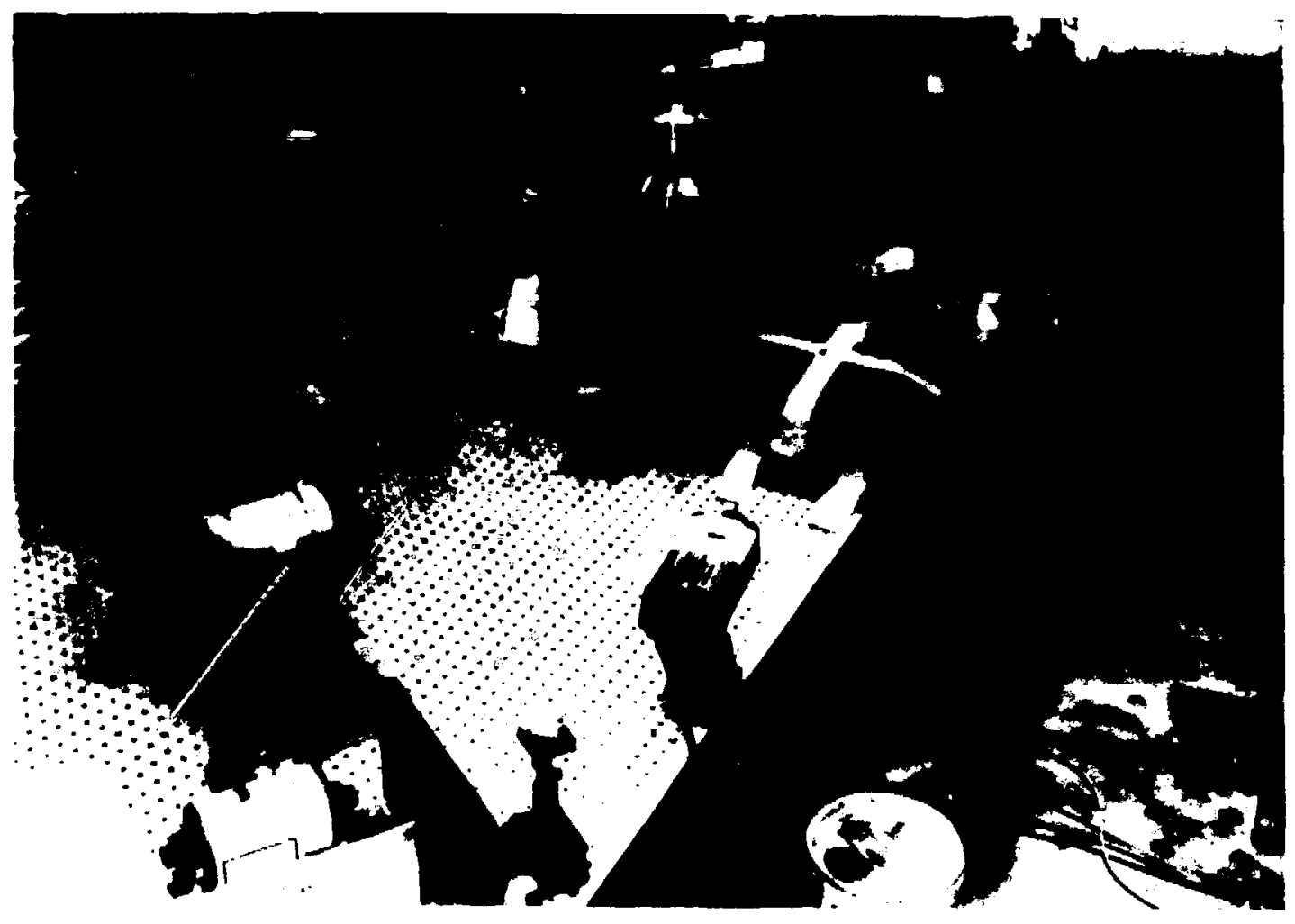

Figure 15. Optical Bench Photograph 
which reflects the beam through a pair of circular beam shaping apertures ("gun sights") to the bottom of the chamber. Here another 90 degree turning prism directs the laser beam directly up into the chamber through the bottom window and onto the spider. The rotating spider directs the laser beam horizontally across the $\mathrm{H}^{-}$ion beam at the interaction point.

All prisms are fabricated from fused silica (Suprasil 1). Ordinary optics are rapidly destroyed by the intense short wavelength radiation. High quality multilayer dielectric mirrors will survive the laser beam and are satisfactory for a single wavelength . Aluminized or micromachined aluminum mirrors are damaged very quickly. The need for operation at several different wavelengths requires fused silica prisms rather than mirrors. The entire apparatus is contained in a tentlike laminar flow clean room. Without the clean room the optical elements would rapidly suffer damage due to the interaction of laser light with any dust particles setting on them.

\section{E. Magnet Configurations}

Figure 7 showed the particle trajectories in a highly simplified manner, indicating only paths for incident $\mathrm{H}^{-}$ions. The "H- beam," while predominantly $\mathrm{H}^{-}$ions, also contains electrons, protons, and neutral hydrogen atoms. These particles all travel with the same velocity ( $B=$ 
.842). Species other than $\mathrm{H}^{-}$are normally produced by gas stripping, that is, collisions of the $\mathrm{H}^{-}$lons with residual gas molecules in the long beam transport system. The magnet configuration must sweep these gas-stripped species away from the detectors in order to prevent the gas stripped signal from completely obscuring the desired photodetachment signal.

Normally gas stripped products are present in very sma11 quantity. However when an alignment phosphor is inserted upstream of our apparatus the entire beam is stripped to $\mathrm{H}^{+}$. When this occurs the magnets must direct the stripped component safely into the beam dump which is the only area shielded well enough to absorb the full beam current without producing excessive radiation levels. The primary function of the magnets is, of course, to direct the photodetachment fraguents $\left(e^{-}, \mathrm{H}^{\circ}, \mathrm{H}^{+}\right)$into their respective detectors.

The above objectives were accomplished by the magnet configuration shown schematically in figure 16 . This is a top view of the apparatus with the field lines of the upstream magnet directed vertically into the paper. The identical downstrean magnet is reversed to compensate the beans. The upstrean nagnet sweeps gas stripped electrons out of the bean and provides separation of the $\mathrm{H}^{\mathrm{O}}$ and $\mathrm{H}^{+}$contanination from the desired $\mathrm{H}^{-}$ion beam. The 


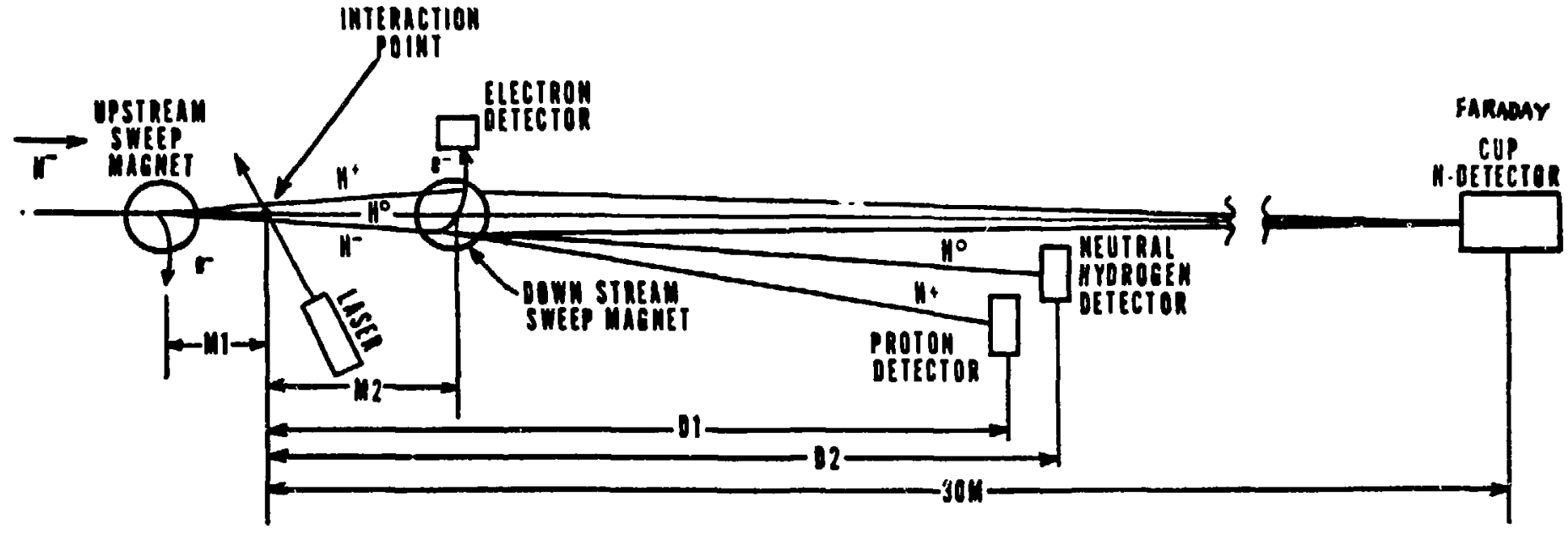

Figure 16. Magnet and Particle Detector Geometry 
downstream sweeping magnet recombines these three beams so that they converge into the Faraday cup in the shielded beam dump area.

When photodetachments occur in the interaction region, $\mathrm{H}^{-}$ions become $\mathrm{H}^{\circ}$ or $\mathrm{H}^{+}$, between the magnets. When this occurs the downstream magnet no longer compensates for the upstream magnet. The particles are instead directed into their respective detectors.

The electrons, having small mass, are swept directly out of the beam at 90 degrees and into the electron detector. The result of single electron photodetachments are $\mathrm{H}^{\mathrm{O}} \mathrm{s}$. These are undeflected by the downstream magnet. The $\mathrm{H}^{\mathrm{O}}$ 's continue along their original uncompensated path until they strike the $\mathrm{H}^{\circ}$ detector. The protons which result from two electron photoejection are deflected to the right by the downstream magnet. This deflection, together with the deflection (as $\mathrm{H}^{-}$ions) from the upstream sweeping magnet, gives them twice the angular deflection of the $\mathrm{H}^{\mathrm{O}} \mathrm{s}$.

It should be noted that any particle which is gas stripped between the two magnets will be directed into the detectors. Coincidence gating with the narrow laser flash minimizes, but does not eliminate, noise due to this gas stripping. For this reason, the length between magnets $\left(M_{1}+M_{2}\right.$ in figure 16) is minimized. Even so, gas 
stripping is a major source of background in the two electron photodetachment measurement.

Another process produces counts in the $\mathrm{H}^{+}$detector which do not correspond to true two electron photodetachment. This is electric field ionization of highly excited $\mathrm{H}^{\circ} \mathrm{s}$ in the downstream magnet. When single electron photodetachment produces $\mathrm{H}^{\circ} \mathrm{s}$ in highly excited states they may be field ionized while passing through the downstream magnet and thus deflected into the $\mathrm{H}^{+}$detector. The very large electric field which strips the excited atoms is of course due to Lorentz transformation of the magnetic field into the atom's rest frame. The electric field is large (up to $2 \mathrm{MV} / \mathrm{cm}$ ) due to the extreme velocity of the particles.

The c.ceshold for field stripping to occur varies as the inverse fourth power of the field, that is $n_{c} \sim \frac{1}{B^{4}}$ where $n_{c}$ is the principal quantum number of the lowest $H^{\circ}$ state which can be stripped by a nagnetic field of strength B (Bayfield 1979). For any given strength of magnetic field $\mathrm{H}^{\mathrm{O}} \mathrm{s}$ with principal quantum number $\mathrm{n}$ less than this critical value will not be stripped. Ho's generated (by photodetachment of $\mathrm{H}^{-}$) in excited states with $n$ greater than or equal to $n_{c}$ will be stripped and seen in the proton counter along with the true two electron photodetachment signal. This phenomonon causes 
almost no degradation of the one-electron channel but can seriously distort the two-electron signal.

The field stripping effect was not considered in initial design of the experiment. A large deflection field of 3600 Gauss was employed on the sweeping magnets. This insured reasonable deflection of the photodetached beams in a flight path of a few meters. Initial observations revealed the stripping signal.

Throughout the course of the experiment efforts were directed at reducing the field on the sweeping magnet in order to minimize the contamination of the true twoelectron photodetachment signal. In the final hours of "beam time" authorized for the experiment, we were able to reduce the sweep field to 22 Gauss. At this low field only states with $\mathrm{n}$ greater than 13 were stripped. The signal due to field stripping was reduced to a value 15 times smaller than the maximum two electron photodetachment signal. This small perturbation was treated as a constant background above $14.35 e v$ and subtracted from the two-electron signal. The two-electron signal was thus distinct except very near the threshold.

The data with high magnetic fields actually gives valuable information about single electron photodetachment channels into the higher excited states. The use of various stripping fields allows branching ratios and 
channels into specific states to be observed. Oneelectron photodetachments into Rydberg states have been described as a mirror image of the two electron threshold (Temkin 1980). The field stripping effect allows these channels to be observed selectively. The field stripping phenomenon is discussed in more detail in the next chapter.

Five different magnetic fields were employed in the course of the experiment. Each field provides unique data on channels into the higher excited states. Three different pairs of magnets, designated $A, B$, and $C$, were employed to achieve these fields. In each case operation is similar to that depicted in figure 16.

Table 4 lists the pertinent characteristics for each configuration. In some cases one detector is not available due to physical constraints. The available signals are indicated by check marks. The configurations are identified by the peak magnetic field which causes stripping. The lowest $\mathrm{H}^{\mathrm{O}}$ quantum state which undergoes stripping is indicated by its $n$ value for each field. The dimensions indicated are keyed to symbols on figure 16.

The 380 Gauss configuration is basically the same as that employed in previous experiments $(200,323,339)$ with the addition of $\mathrm{H}^{\circ}$ and $\mathrm{H}^{+}$detectors. These detectors must be mounted approximately 20 meters downstream to get adequate defiection of the heavy particles $\left(\mathrm{H}^{\circ}\right.$ and $\left.\mathrm{H}^{+}\right)$with 
Table 4. Magnet Configurations.

\begin{tabular}{c|c|c|c|c|c|c|c}
\hline $\begin{array}{c}\text { Magnetic } \\
\text { Field } \\
\text { Gauss }\end{array}$ & $\begin{array}{c}\text { Detectors } \\
\mathrm{e}^{-}\end{array}$ & $\mathrm{H}^{\mathrm{O}}$ & $\mathrm{H}^{+}$ & $\begin{array}{c}(\mathrm{B} . \mathrm{d} \mathrm{d} \\
(\text { Gaus }-M)\end{array}$ & $\begin{array}{c}\text { Lowest } \\
\text { Ho state } \\
\text { ionized } \\
\mathrm{n}_{\mathrm{c}}\end{array}$ & $\begin{array}{r}\mathrm{D}_{1} \\
(\mathrm{M})\end{array}$ & $\begin{array}{r}\mathrm{D}_{2} \\
(\mathrm{M})\end{array}$ \\
\hline 22 & & $\mathrm{X}$ & $\mathrm{X}$ & 48 & 14 & 20.8 & 21.4 \\
\hline 380 & $\mathrm{X}$ & $\mathrm{X}$ & $\mathrm{X}$ & 53 & 7 & 20.8 & $21 .$. \\
\hline 780 & & $\mathrm{X}$ & $\mathrm{X}$ & 110 & 6 & 20.8 & 21.4 \\
\hline 2200 & $\mathrm{X}$ & & $\mathrm{X}$ & 410 & 5 & 2.67 & $\mathrm{X}$ \\
\hline 3600 & $\mathrm{X}$ & $\mathrm{X}$ & $\mathrm{X}$ & 660 & 4 & 2.05 & 2.67 \\
\hline
\end{tabular}


the small magnets employed. The old scheme has been previously described (Bryant et al 1977). The 780 Gauss configuration is identical. Increased magnet current gives 780 Gauss field. The $\mathrm{H}^{\circ}$ and $\mathrm{H}^{+}$detectors at 20 meters are moved out to twice their previous spacing from the beam line to compensate for the increased field. The electrons do not strike their detector with this increased field.

In the two highest field configurations ( 3600 Gauss and 2200 Gauss), two sets of cross field magnets are used. A 380 Gauss magnet sweeps the electrons into the electron detector. The protons are deflected vert..cally by a second set of powerful magnets located farther from the scattering chamber. The dimensions and fields given in table 2 are for the larger proton sweeping magnets.

Magnets A, B, and C (see figure 17) are electromagnets with copper conductors. Magnets A are air cooled with soft (Armco) iron cores. The two magnets each have pole tip diameter of $14 \mathrm{~cm}$ and gap of $7 \mathrm{~cm}$. The maximum field of 780 Gauss with 5 Amperes current is limited by heating rather than core saturation. These same magnets were used in previous experiments and are described in more detail elsewhere (Sharifian 1977).

Magnets $B$ are similar in design but larger. They are cooled by flowing water through the conductors which are hollow rectangular tubes made of copper. These magnets 


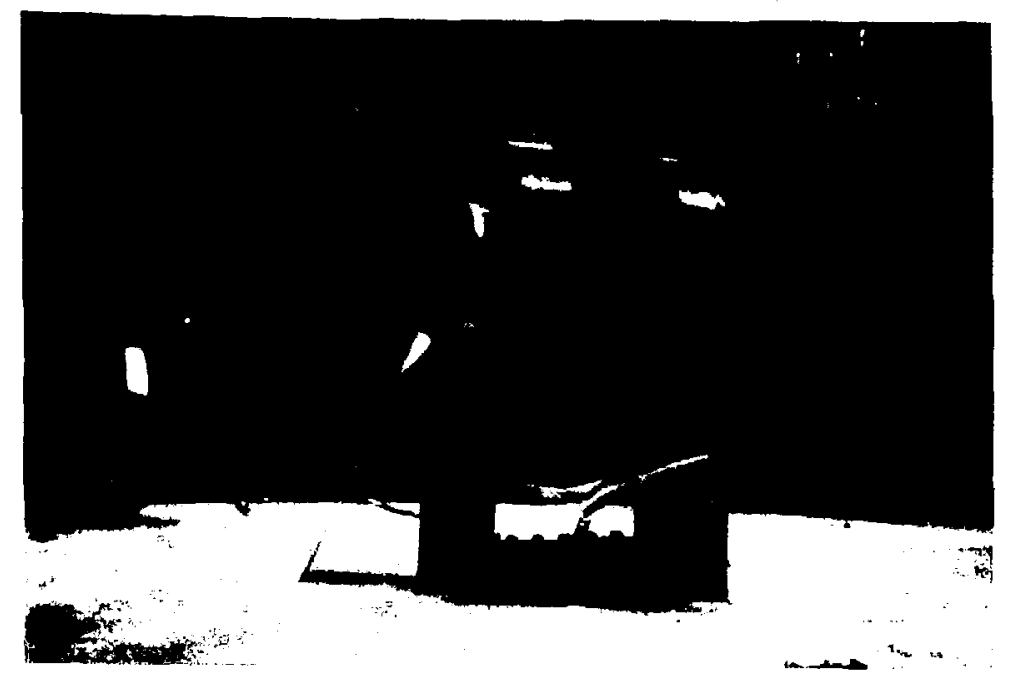

A

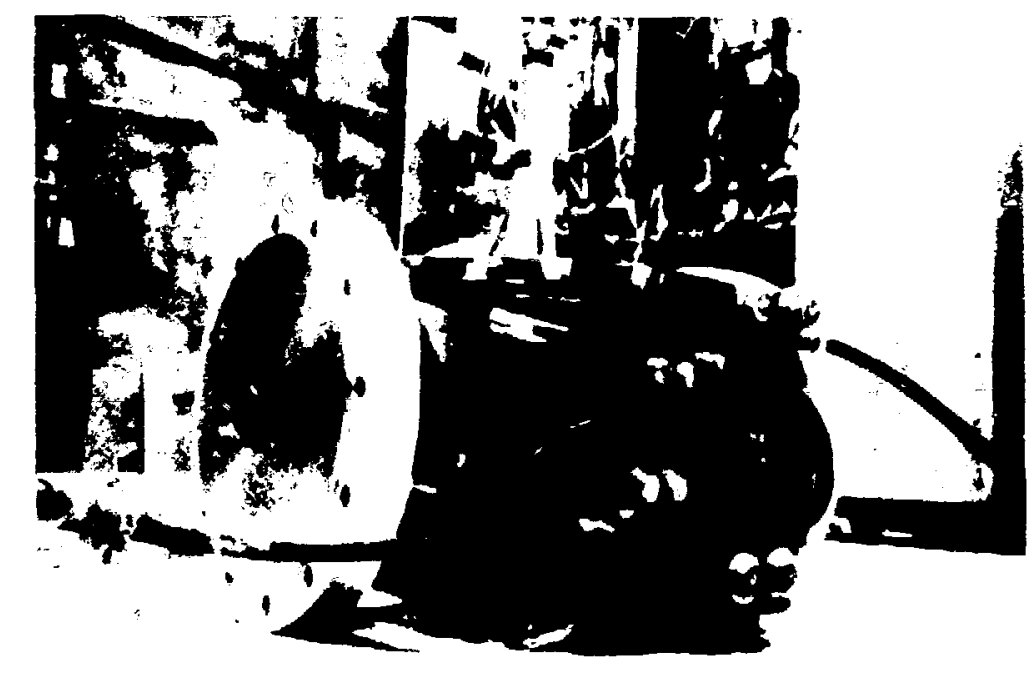

B 
have rectangular pole tips with an effective length of $18.3 \mathrm{~cm}$. The magnet gap is $3.8 \mathrm{~cm}$. A field of 3600 Gaus corresponds to a current of 300 amperes. Somewhat more field can be obtained with increased current.

Downstream magnet $C$ for the 22 Gauss configuration is of a somewhat different construction. For the upstream magnet 380 Gauss is employed using magnet $A$. The downstream magnet is an air core design consisting of a pair of wires taped to the left and right side of the 6 inch diameter stainless steel beam pipe. Currents flowing in opposite directions along the wires produce fields which add vectorally to give a vertical field at the center of the nonmagnetic beam ixe. The length of the wires is such that the product of field times length is equal to that of the 380 Gauss magnet upstream. Compensation is thus obtained. The 22 Gauss field is achieved by passing a 500 ampere current through the wires which are number 0000 gauge copper welding cable. The electron signal is not available with this configuration. Trajectories for the relativistic particles through the various magnetic field configurations were originally calculated using numerical procedures to track the particles over circular paths (cyclotron orbits) inside magnets and straight lines outside. This is the so called "Hard Edge" model. During setup, fine tuning of both 
magnet currents and detector position was employed to peak the counting rate in each detector. A Hall effect Gauss meter was used to continuously monitor the field. This allowed resetting magnets accurately despite core hysteresis. Magnet current was also monitored using calibrated shunts.

While setting the magnets and detector positions is simple in theory, it is no small task in practice. The $\mathrm{H}^{-}$ion beam with $2 \mathrm{~mm}$ diameter is surrounded by a non-Gaussian halo which extends out to approximately $2 \mathrm{~cm}$. The detectors must be outside this halo region or they will be swamped with noise. In order to obtain sufficient separation of the photodetached beams from the main beam, a very long flight path is required for low field measurements. The long "lever arm" translates small angular misalignments of the beam into large spatial motions on the detectors. Moving detectors to compensate is time consuming. The beam must be shut off and a radiation survey conducted whenever the experimental area is entered to adjust detectors. Because of these complications several hours or even days of beam time were required to tune up each configuration.

\section{F. Sensors}

Various sensors are used to measure the quantities required for the cross section calculations. They fall 
into three convenient groups; particle detectors, photon detectors, and beam current monitors. There are also numerous other sensors including shunts used to measure magnet current, a Hall effect Gauss meter, the angle encoder and radiation monitors employed to measure personnel radiation exposure.

Fast scintillation detectors are used to count the number of photodetached $\mathrm{H}^{\mathrm{O}} \mathrm{s}$ and $\mathrm{H}^{+} \mathrm{s}$. Two identical units (see figure 18) are used. Each detector consists of a small block of plastic scintillator material (Pilot F) optically coupled to the face of a 2 inch photomultiplier tube (PMT) by a 6 inch length of ultraviolet-transmitting lucite. The lucite rod acts as a light guide, transmitting the scintillation flash to the PMT.

The scintillator is mounted inside a metal cup made of 16 gauge stainless steel tubing. The cup is attached to a standard vacuum flange by a stainless steel bellows mount. This allows the cup containing the scintillator to be inserted into the 6 inch beam pipe. The inside of the cup is at atmospheric pressure. Three threaded rods allow adjustment of the distance between the scintillator and the beam center line. These are adjusted so that the scintillator intercepts the desired photodetached beam.

Due to their high energy the photodetached $\mathrm{H}^{+}$and $\mathrm{H}^{\circ}$ particles penetrate the thin walls of the cup to excite 


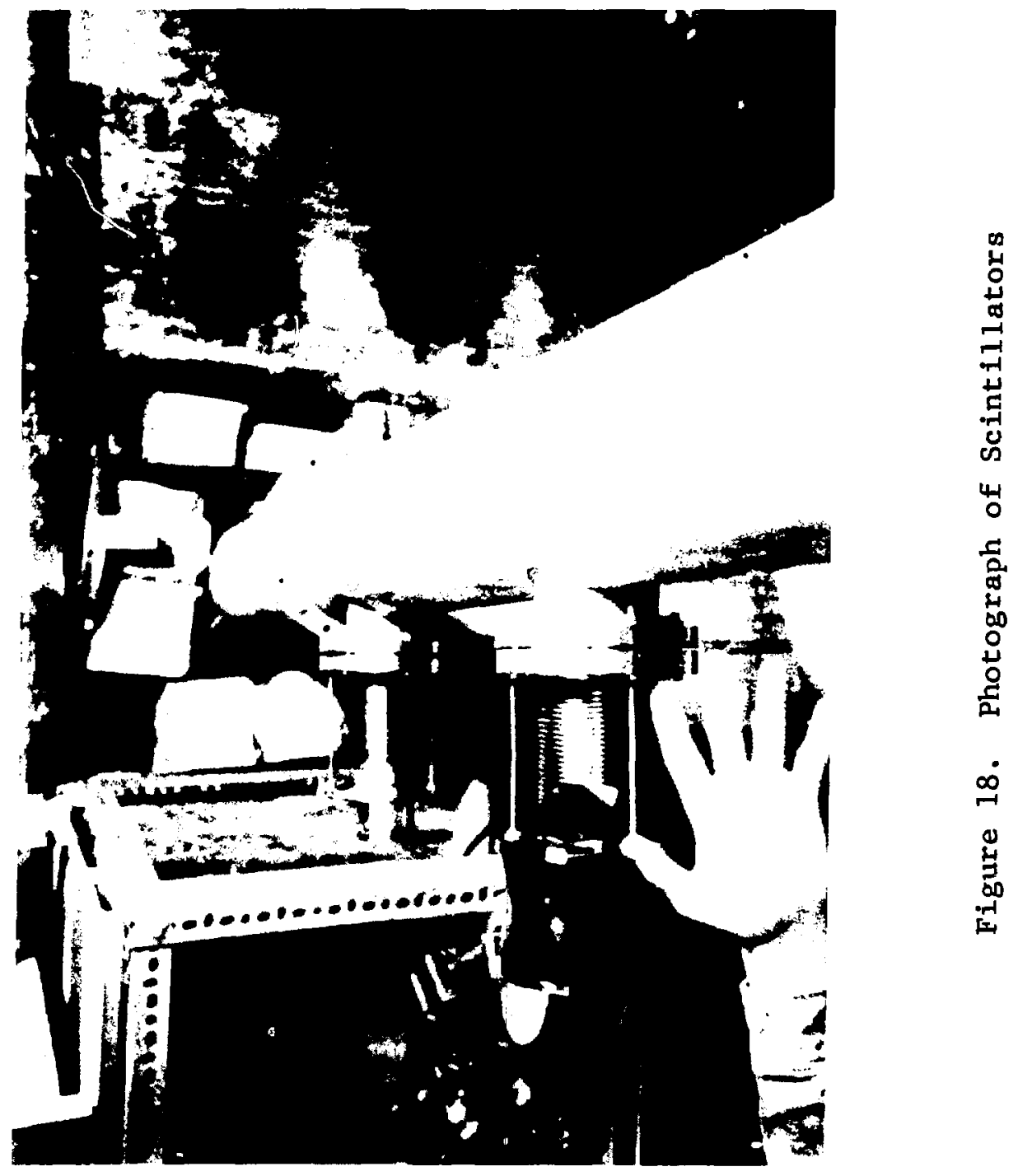


the scintillators. The particles pass completely through the scintillator material. In the process they deposit a fraction of their energy. When multiple photodetacinents occur, each particie deposits an equal amount of energy. Thus the scintillation flash and the PMT output pulse are proportional to the number of photodetachments which occurred for the laser pulse.

Actually there is a statistical fluctuation (Landau fluctuation) in the energy deposited when a particle passes through the scintillator. In a thin detector landau fluctuations occur when the energy lost by a charged particle is small compared to its total energy (Landau 1944). These Landau flucuations degrade the energy resolution of the detector somewhat. The result is observed on a pulse height spectrum as a slight smearing out of the discrete peaks due to multiple photodetachments. The Landau fluctuations do not significantly affect the measurement since the total number of particles counted is large.

The scintillation flash is detected by a PMT. The PMT is mounted in a LAMPF standard base. The output pulse has an approximately 3 ns risetime with a width of less than $10 \mathrm{~ns}$ into a $50 \mathrm{ohm}$ load. The narrow pulse width allows extreme timing accuracy and high background counting rate providing the ability to operate with high beam current. 
The PMT can provide a large current pulse (up to $100 \mathrm{~mA}$ ) for a short time. We are thus able to count a large number of simultaneously photodetached particles. The bias voltage is adjusted so that a single particle gives 20 millivolt output into 50 ohms. 100 particles should then give approximately 2 volts. High background rates can cause droop due to high average current. This droop is not generally a problem.

The electron scintillator is similar in concept and design. Here the plastic scintillator must be mounted in the vacuum. The photodetached electrons with $B$ of 0.842 have only $435 \mathrm{keV}$ of kinetic energy as opposed to $800 \mathrm{MeV}$ for the $\mathrm{H}^{\mathrm{O}} \mathrm{s}$ and $\mathrm{H}^{+} \mathrm{s}$. The scintillator is a 2 inch disk of pilot F plastic scintillator material glued directly to the face of the LAMPF standard PMT assembly. The face of the disk is covered on the vacuum side by a thin aluminum foil which blocks laser photons which would otherwise be detected by the PMT. The foil also acts as a barrier to preveni contamination of the vacuum by outgassing from the plastic. The 3 mil foil thickness causes negligible loss of energy resolution.

The electrical details of the electron detector are Identical to the $\mathrm{H}^{\circ}$ and $\mathrm{H}^{+}$detectors except that higher bias voltage is employed to give comparable output. Bias is adjusted to give 20 millivolts output per electron 
detached. There are no Landau fluctuations since the electrons are totally absorbed in the plastic due to their short range.

For some of the measurements electrons were detected with a silicon solid state detector (SSD). The SSD detector system is characterized by a very slow response time. The solid state detector is in theory capable of extreme energy resolution. Such energy resolution is not realized in practice due to the slow response of the system. Very low beam current would be required in order to obtain the high resolution potential of the SSD. For currents high enough to obtain reasonable counting rates, the scintillation detector actually provides better energy resolution.

The solid state detector (Ortec model TR-22300-1000) consists of a wafer of n-type silicon over which a thiil layer (a few microns) of p-type silicon is produced by doping from the gas phase. A thin (much less than the range of $435 \mathrm{kev}$ electrons) aluminum film is deposited over both faces on the wafer to serve as front and back electrode and to block visible photons from entering the unit.

A high reverse bias voltage is applied across the junction. The reverse bias creates an electrically insulating depletion region of approximately 1000 micron 
thickness at the junction and extending into the substrate. The range of $435 \mathrm{keV}$ electrons (see figure 19) is less than 1000 microns. Since the depletion region extends from the surface to a depth of 1000 microns, the incident electrons lose all their kinetic energy within the barrier. This type of SSD is referred to as a surface barrier detector since the depletion region (barrier) occurs near the surface.

When the charged particles are absorbed in the depletion region, they create free electron-hole pairs. Each $3.6 \mathrm{eV}$ of energy loss creates one electron-hole pair. This rate is almost independent of particle energy or species, provided that the electric field is sufficient to separate the charge carriers before they are lost to recombination.

For $435 \mathrm{keV}$ electrons the rate of charge production is approximately $4 \times 10^{-14}$ Coulombs per particle. The charged particles are collected by the bias voltage. The current pulse thus produced is amplified with an integrating preamplifier or charge amplifier.

While the liberation of charge and ensuing current pulse is prompt, the long integrating time required to smooth out shot noise and thermal noise produces a slow output pulse in the electronics. The electronics associated with this detector is discussed in the following section. 


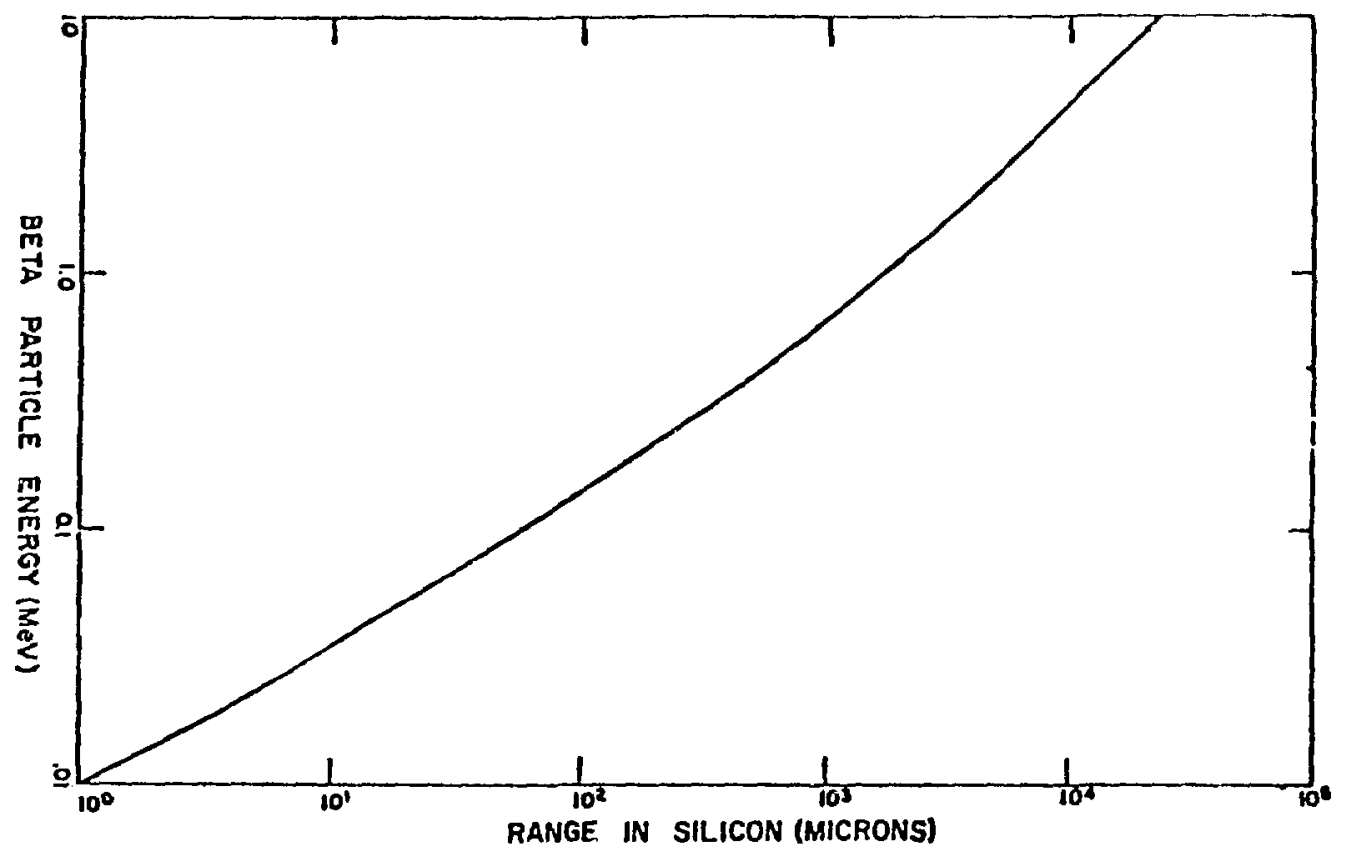

Figure 19. Beta particles range in silicon. After ORTEC Instruction Manual for surface barrier detectors. 
Two types of photon detectors are employed; solid state photodiodes and planar vacuum photodiodes. The solid state photodiodes are EGG type UV-100. These units are sensitive throughout the range of laser photon energies available. The sensitivity is however strongly dependent on the wavelength. Sensitivity is maximum for the laser fundamental which is in the near IR. The operation of these units is identical to the solid state electron detector except that the charged pairs are liberated by incident photons. The photodiodes are reverse biased by a small 28 volt battery.

The silicon photodiodes have approximately 3 ns rise time. This gives an accurate reproduction of the 8 nanosecond laser pulse envelope but does not reveal the mode spiking .

Two solid state photon detectors are employed. One, referred to as "external SSPD," is located on the optical bench. This unit views scattered 1ight from the 90 degree turning prism giving indication of laser power rather than power inside the chamber. Figure 8 shows the SSPD location on the bench.

The other SSPD is located inside the chamber sampling the photon beam that actually crosses the $\mathrm{s}^{-}$beam. This unit is located in the photon beam catcher whilh is attached to the rotating spider arm. The beam catcher 
consists of a cone machined into a block of aluminum. The shiny surface of the cone funnels the laser beam down through a small aperture into a cavity. The laser energy is dissipated in the cavity by multiple reflection losses. Since the losses are distributed over the entire inside surface of the cavity, damage from the high energy laser is minimal.

The photodiode views the inside of the cavity through a very small aperture, sampling a fraction of the laser energy. The funneling cone and cavity absorber of the laser beam catcher are extremely effective in preventing the reflection of laser photons back across the $\mathrm{H}^{-}$ion beam. Such backwards reflections could produce significant background since they would be Doppler shifted to a different effective energy than the incident photons. The planar vacuum photodiodes (VPD) have 0.5 nanosecond response time into a $50 \mathrm{ohm}$ load. The fast response time of these units reveals the mode spiking when it occurs. The photodiodes have S1 photocathodes with quartz windows and provide useful response throughout the wavelength range of interest. The sensitivity is however wavelength dependent. The photodiodes are forward biased at 1000 volts to prevent saturation due to space charge accumulation.

A calorimeter type power meter is also employed for occasional calibration of the photon sensors. Although 
absolute calibration is not required, linearity is essential.

Two types of beam current monitors are employed. The primary reference is the Faraday cup which consists of a massive $(700 \mathrm{~kg}$ ) graphite cup of sufficient mass to completely stop the incident protons and prevent reenission of any charged particles produced in collisions. The $\mathrm{H}^{-}$ions are totally stripped to $\mathrm{H}^{+}$by a thin foil before reaching the Faraday cup. The cup then receives one unit of positive charge for each incident charged particle. The charge is drained off the Faraday cup by a teflon insulated wire and led to the input of a sensitive charge amplifier. The output of the charge amplifier provides a voltage to frequency conversion. The calibration is set so that an output of 10 Hertz corresponds with a beam current of 1 nanoamp.

The entire unit is linear and accurate to better than 1 percent absolute (Barrett 1975). The Faraday cup is slow in response requiring approximately 1 second to fully respond to changes in beam current. Fast fluctuations in beam current and variations of current within the macropulse are typical at LAMPF. In order to properly normalize the cross section against these fast fluctuations a fast ion chamber is employed.

The fast ion chamber consists of a number of thin metal plates. The space between the plates is filled with 
hydrogen gas. When high energy protons pass through the gas, Ionization occurs as the protons lose energy to the gas. A high potential is applied across the series connected plates producing an electric field in the gas. The field collects the free electrons producing an output current which is proportional to the proton beam current. The fast ion chamber and its associated charge amplifier provides a response time of approximately 10 microseconds. This is adequate to reflect variations in beam current within the macropulst.. The fast ion chamber signal is digitized in time with the laser flash giving the current at the instant the laser is fired.

G. Electronics

The photodetachment experiment involved a highly complex array of fast electronic equipment. Figure 20 shows some of the circuitry which completely fills 7 equipment racks, each 6 feet high. This complexity is required to optimize data collection rate. With limited beam time available, this is necesary. It is a tribute to the resourcefulness of the research team that all of this complex and rather hastily constructed equipment was made to operate successfully for the duration of the measurements.

The complexity of the setup precludes presentation of circuit diagrams or detailed circuit description of each 


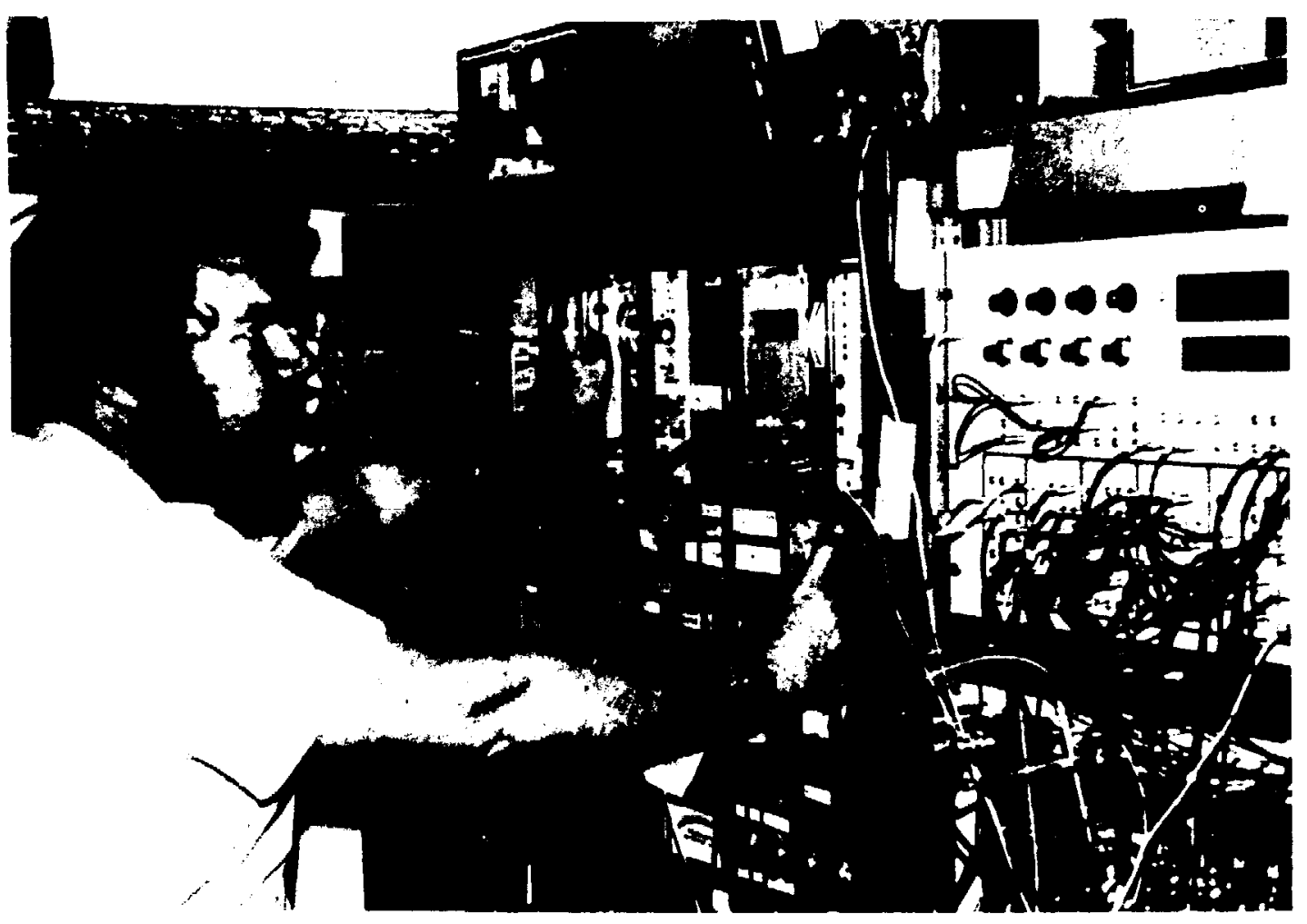

Figure 20. Photograph of Part of Fast Electronics Setup 
unit. Many of these units are stanc rd NIM or CAMAC modules. Characteristics of these modules may be found in the manufacturer's manual. nere I will present an overview of the data acquisition system using block diagrams and emphasizing concepts rather than details. It is hoped that sufficient information will be presented to allow duplication of the apparatus as well as critical evaluation of the validity of the data collected using it.

Figure 21 shows a highly simplified electronics block diagram. On the left-hand side are the various sensors as well as the laser. All of these units are located in the experimental area, access to which is restricted due to high radiation levels when the beam is on. Sensors include particle counters, photon monitors, beam current monitors and an angle encoder. The other systems shown are located in the data acquisition trailer.

The laser trigger generator produces flashlamp and Qswitch triggers for laser timing. The triggers are generated in synchronization with the accelerator timing pulses. This insures that the laser will fire in the center of beam current macropulses. All other systems are synchronized to the laser flash. The fast photon detector (vacuum photodiode) output pulses are used to trigger the coincidence circuits and other fast timing allowing large jitter between laser trigger and output flash to be tolerated. 


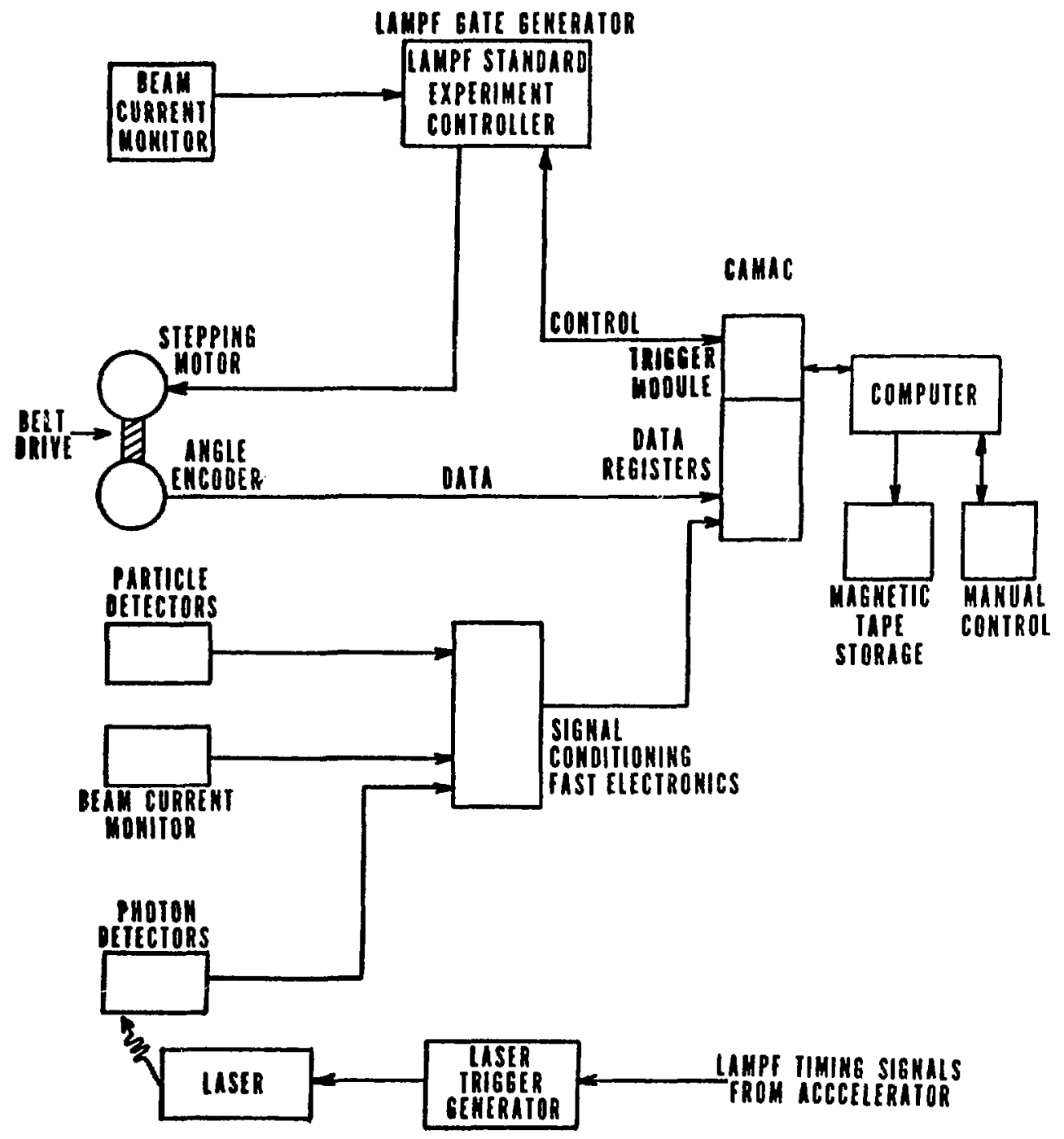

Figure 21. Electronics Block Diagram 
The signal conditioning and fast electronics occupies several NIM bins. Here coincidence circuits provide output pulses when particle detector pulses occur in time with the laser flash. Analog signals are attenuated or amplified for optimum level. Signal inversion and pulse shaping are also employed to generate pulses of proper level, polarity, and temporal characteristics for digitization. Signals are delayed as required to center al1 pulses in digitization and coincidence windows. Communication with the computer is via CAMAC units. Each of these units contains output registers which are interrogated by the computer after every laser flash.

The coincidence pulses are applied to scalers. Fast scalers also count ungated scintillation flashes for background, accidental counts, laser flashes, beam pulses, clock pulses, and numerous other quantities. A special set of registers relays angle encoder reading as an 18 bit binary word. Analog to digital converters (ADCs) are used to read all analog signals. These ADC's are of two types: peak sensing and integrating. For both types the input voltage is sensed only during an externally applied ADC strobe pulse. The peak value or the time integral of the input is digitized by the two types respectively. The scalers and ADCs are multichannel units, most having 12 inputs. In total approximately 40 channels are read. 
The first channel is read by the computer several hundred microseconds after each laser firing. Each of the other channels is then read sequentially. The process is repeated for each laser flash.

The digital words representing $\mathrm{ADC}$ levels and register contents are recorded on magnetic tape after each laser flash. At the end of each angle a software sum which gives the total number of scaler or ADC counts that occurred during the angle is recorded on a computer disk. These software sums are used for the calculation of cross section.

Computer control signals are passed through CAMAC modules in the form of event triggers and register contents. The computer, however, exerts minimal control over the experiment. In fact the entire experiment may be run manually. The essential computer scalers are duplicated with visible scalers having LED readout. These visible scalers are reset at the end of each angle rather than after each laser burst as ocsurs for the computer scalers. In manual operation ADC function can be duplicated by pulse height analyzers. Manual operation has only been employed as a diagnostic because the time required for writing down all scaler sums after each angle limits the data collection rate to an unacceptat le level in this mode. 
The "end-of-an-angle" is initiated when a preset quantity of charge has passed through the apparatus. This is accomplished by integration of the Faraday cup signal. A preset comparator scaler in the LAMPF experiment controller generates a pulse when this condition occurs. The pulse initiates the advance of the stepping motor and signals end of angle to the computer.

The LAMPF standard experiment controller is officially designated as "LAMPF gate generator." The LAMPF gate generator provides necessary gating signals (see figure 22), and performs a number of control functions. Many NIM units (logic modules) are gated to respond only when a beam current macropulse exists. This is accomplished by use of the beam gate signal, a 600 us pulse which is high for the duration of the beam current macropulse. The beam gate is generated in the LAMPF accelerator control room and is arranged to drop out whenever beam current $\left(\mathrm{H}^{+}\right.$or $\left.\mathrm{H}^{-}\right)$is absent. The LAMPF gate generator distributes this pulse to the logic units to prevent their operation when the beam is off or between beam macropulses. Hence its name (Gate Generator).

This unit also generates an experiment run gate which is used to gate logic units on when the experiment is running. When the run gate is low, all data acquisition stops. The run gate is low between angles (while the stepping motor is moving the spider), between runs, when 


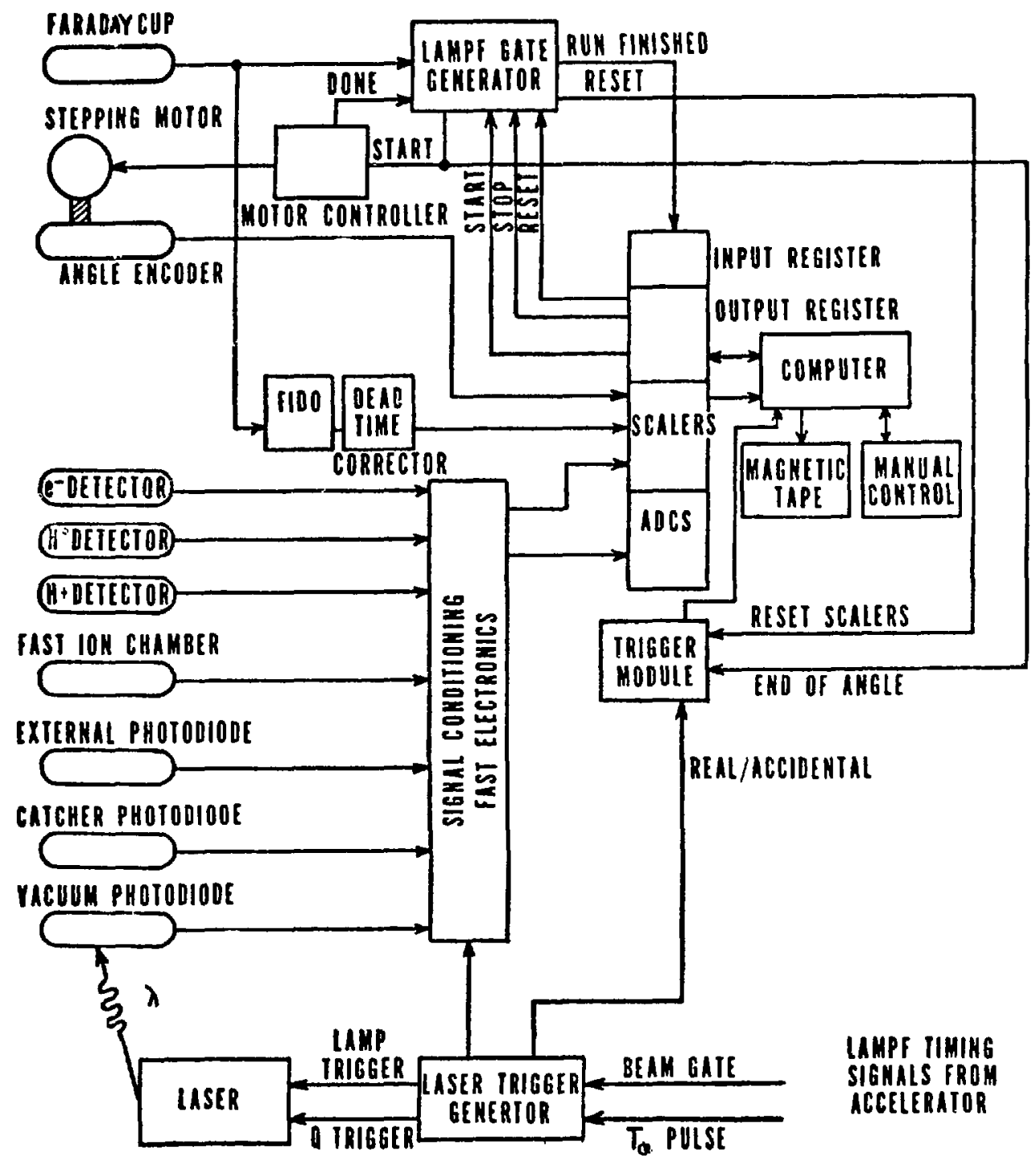

Figure 22. Electronics Control Diagram 
beam current is off for prolonged periods, and any time the experimenter wishes to halt temporarily the experiment. The run gate is controlled by start and stop commands. These may be activated by pushbuttons on the LAMPF experiment controller but are normally controlled automatically. The stop command is generated whenever angle is changed, or when a run of preset number of angles is finished. The start command is generated by the conputer when a run is started. The LAMPF gate generator also allows clearing all scalers by the reset function which may be activated by front panel pushbutton switch or computer control. These control pathways are designated on figure 22 .

Also indicated in figure 22 is the stepping motor controller. Advance motor commands are generated by the LAMPF gate generator whenever the preset quantity of charge is sensed. This condition is called "end of angle". Upon receipt of a start trigger, the stepping motor controller sends a preset number of pulses to the motor. The number of steps is set with front panel thumbwheel switches on the controller unit. When the preset number of pulses has been transmitted, the controller issues a "done" command allowing data acquisition to resume for a new angle. The done commands are counted in a preset scaler called the angle counter. When the 
desired number of angles has been counted, the run is terminated. At this time cross sections are calculated and displayed graphically.

Associated with the Faraday cup current monitor are two circuits "watch Dog" and dead time corrector. The watch dog circuit automatically interrupts data acquisition whenever beam current is absent or below acceptable levels for a period exceeding 15 seconds. This function is accomplished by blocking off the run gate. The dead time corrector compensates for the dead time which occurs while the computer reads scalers. The Faraday cup current monitor produces a pulse train whose frequency depends linearly on beam current. The total charge is calculated by counting these pulses which are asynchronous with experiment operation. The number of pulses counted must be corrected for the dead time which occurs while the run gate is off during "computer busy."

H. Signal Digitization

Due to the high laser power employed, multiple photodetachments generally occur. That is, more than one simultaneous particle count occurs per laser flash. If only scalers were employed, the counts would be limited to a maximum of one count per laser flash. This would not reflect true cross section.

This problem is circumvented by digitization of analog signals from particle detectors with a linear 
integrating analog to digital converter (ADC). The scaler counts, however, are useful for tune up. They are also valid in region of very low cross section or when low laser power is employed. All analog signais are digitized in a similar manner.

Figure 23 shows the particle counting electronics in detail. The fast vacuum photodiode (VPD) produces narrow trigger pulses (approximately $10 \mathrm{~ns}$ FWHM) which correspond to the laser flash. After a cable delay of approximately 100 nanoseconds, these pulses trigger discriminator D1. The input attenuator (PAD) must be chosen so that the discriminator triggers reliably throughout the range of laser operating powers without "double pulsing" from small cable reflections.

The 100 nanosecond wide pulse from D1 is counted on visible and computer scalers giving the number of laser flashes. The pulse from D1 triggers discriminators D3, D4 and other timing circuits. D3 generates a 20 nanosecond gate (ADC read) which strobes the ADC. D4 generates a 15 nanosecond gate (coin gate) which is used to form coincidence of particle count with laser flash.

D3 and D4 may also be triggered (through the strobe OR gate) by a background trigger which is generated in the laser trigger generator. The background trigger occurs in each beam current macropulse following a laser flash at 


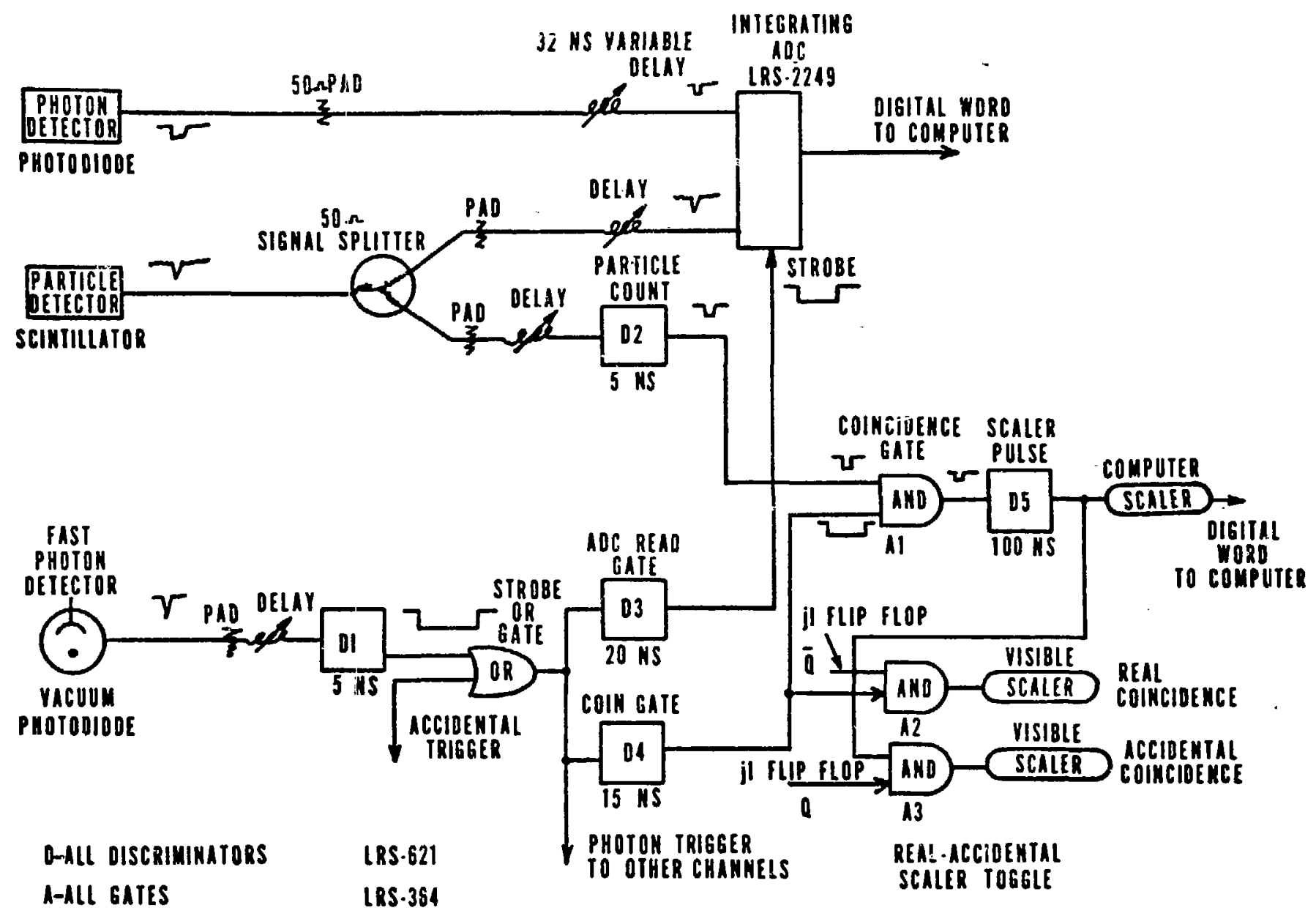

Figure 23. Signal Digitization Simplified Schematic 
the same point in the macropulse as the laser flash in the previous macropulse. Background triggers initiate the reading of background counts. These background counts are subtracted from the coincidences in later data analysis to obtain the number of real events. The events are termed "coins" or "accidentals" for coincldences and background events respectively. The technique of reading coins and accidentals on successive beam macropulses allows the same ADC channel to be employed for both eliminating errors due to imbalance of the gain of the two channels. Extremely small signals may be extracted from background noise with this technique. The background subtraction compensates for gas stripping noise and other events which are not in time with the laser flash.

The integrating ADC (LRS-2249) converts the total absorbed charge during the period of the strobe pulse into a 10 bit binary word which is read by the computer after each coincidence or background event. A pedestal charge is injected into the 50 ohm input stage to establish the operating point in the linear region and provide a DC baseline to minimize drift in the unit. The pedestal is approximately 5 counts out of the maximum range of 1024 $\left(2^{10}\right)$. The maximum range of the unit is either 256 picocoulombs or -2 volt peak. The input noise is approximately 1 millvolt. Due to the limited dynamic range of the 
$A D C$, it is important that all input signals are at the correct level. The ADC has 12 channels. The 3 particle detectors (scintillators) and 3 photon detectors are read by this unit.

The photon channel seen in figure 23 is typical. Pulse inverting transformers are employed as required to give negative input signal polarity to the ADC. The input attenuator (50 ohm PAD) is selected to give a maximum input signal of .5 volt or 100 picocoulombs for a 10 nanosecond duration pulse into the 50 ohm $A D C$. The variable cable delay line is adjusted to center the photon pulse in the 20 nanosecond timing window of the ADC strobe. Three identical channels provide measurement of the photon pulse for each of the three detectors. The output corresponds to the time integral of the photon flux.

Each of the three particle detector channels is similar. The scintillation counters provide a negative pulse approximately 10 nanoseconds in width. The level is approximately 20 millivolts per particle counted. A 50 ohm power splitter divides the signal between analog (ADC) channel and pulse counting channel while maintaining a $50 \mathrm{ohm}$ impedence on all three ports. Impedence match must be maintained for all fast signals to prevent cable reflections. The analog (ADC) channel is identical to the photon channel previously described. 
The energy resolution of the particle channels is sufficient to display the multiplicity of counted particles as separate peaks in the digitized pulse height spectra. Background spectra display peaks at 0,1 , or 2 electrons while real photodetachments may show up to 100 particles photodetached when high laser power is employed. The statistical distribution seems to be Poisson.

In the counting channel, discriminator D2 is triggered by the particle count. The input attenuation (50 ohm pad) is selected so that a single particle count just triggers the discriminator. The 5 nanosecond output pulse of discriminator D2 is combined with the photon timing signal (coin gate), in AND gate Al. The variable delay on D2 input is adjusted to compensate for cable delays and particle transit delay so that photodetachment counts result in the coincidence with photon triggers.

The output of $\mathrm{Al}$ is used to generate a 100 nanosecond pulse for driving scalers. The computer scaler 1s rad after each laser flash. If one or more particles has been photodetached, the running sum of reals in the computer is advanced by one step. The scaler is always cleared after reading. On the following beam current macropulse the scaler is again read. There is no laser flash during this nacropulse since the laser fires at 10 Hertz while the beam pulses occur at 120 Hertz. If a background count 
occurs, the running sum of backgrounds in the computer is advanced by one count. The sum of real events for each angle is calculated by subtracting backgrounds from coincidences for that angle. It is clear that the computer scaler always reads zero or one since it is cleared after each event.

The visible scalers are not cleared after each event but at the start of each new angle. This allows the experimenter to read the total number of counts at the end of each angle. Separate visible scalers are employed for coins and accidentals. A pair of AND gates (A2 and A3) direct the pulses from D5 to the appropriate visible scaler. This flow is directed by the $Q$ and $\bar{Q}$ outputs from the $j / 1$ flip flop which is located in the laser trigger generator. $\bar{Q}$ is high during real events. A set of triggers ( $j$ and 1 triggers) is likewise used to inform the computer of coincidence and background events respectively. The background trigger (input to strobe OR gate) which simulates laser flashes on background events is also generated by the laser trigger generator.

\section{Silicon Detector Electronics}

Some measurements employed a solid state detector (SSD) for electron counting instead of the electron scintillator. In theory the silicon detector is capable of greater energy resolution than the scintillation 
detector. This was never achieved in practice. The SSD electronics is inherently slow in response compared to the scintillator. The slow response causes a modulation of the desired photodetachment signal by the long decay tails of background pulses. Due to the large number of background pulses with random time relation to the desired pulse an amplitude smearing occurs which destroys the high energy resolution cabability of the SSD. At high beam currents the discrete pulse height spectra (indicating multiplicity of photodetachment events) is destroyed.

A detailed description of the circuitry for the SSD will be given since future experiments call for using a germanium SSD for extremely fine energy resolution. The pulse width from the SSD is approximately 5 microseconds compared to 5 nanoseconds for the scintillator. Very low beam current must be employed to prevent interference of background counts with the desired signal, allowing the ful1 encrgy resolution of the detector to be utilized. Counting rate is thus very low.

Figure 24 shows a simplified circuit for use with the silicon detector. Upon absorption of incident $435 \mathrm{keV}$ electrons, a quantity of charge is promptly released by the detector. The total quantity of charge liberated is directly proportional to the number of electrons absorbed. The integrating preamp (Ortec 142) provides an output step 


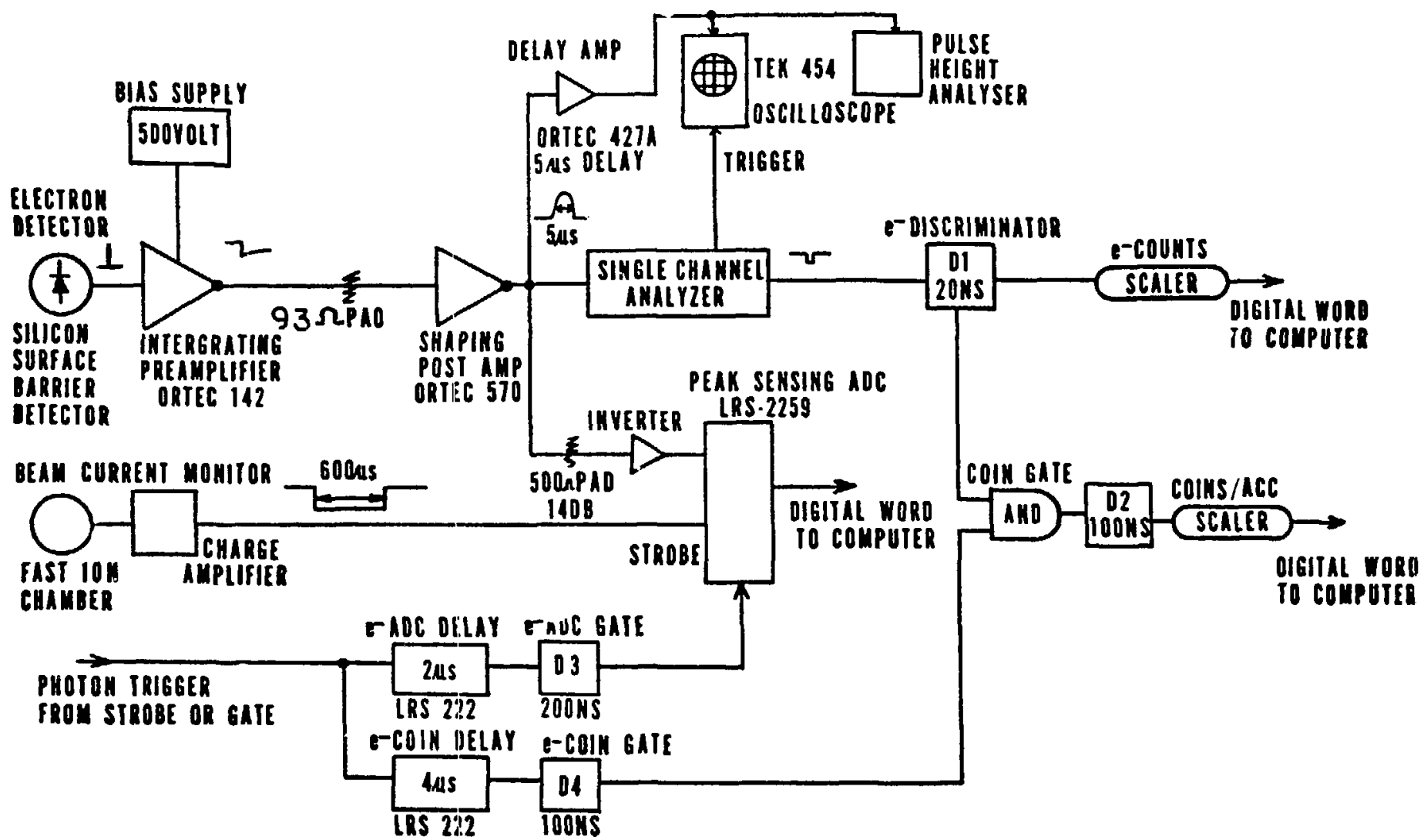

Figure 24. Silicon Detector Circuit 
whose height is proportional to the quantity of charge and thus to the number of electrons. The preamp is attached directly to the detector output connector to minimize capacitive loading on the high impedance input. The minute detector output pulse is comparable to the thermal noise on the preamp input. For this reason a long integrating time is employed to smooth out the noise. The preamp output displays a sharp rise followed by a 300 microsecond decay. This long tail causes pulse pile up under high count rate conditions but is necessary to achieve adequate signal to noise ratio. The preamp has 93 ohm output impedance.

A $93 \mathrm{ohm}$ input attenuator is required on the post amplifier input to prevent saturation. The preamp output is coupled to the data trailer by 130 feet of 93 ohm coaxial cable. The voltage step is applied to a shaping post amplifier (Ortec 570) where the step is shaped to provide a Gaussian output pulse. This method broadens the pulse but is necessary to discriminate against noise. The postamp allows adjustment of the pulse width. In theory the energy resolution is directly proportional to the puise width. We employ minimum pulse width to maximize counting rate. Actually this also improves the energy resolution since spread in pulse height due to excessive background count rate is the major degrading factor. The 
postamp gain is adjusted so that a single electron gives an output of 100 millivolts. This arrangement gives a dynamic range of 100 to 1 . That is, a single electron produces a pulse well above the noise floor while 100 simultaneous counts will just saturate the amplifier at 10 volts.

The Gaussian pulse (5 microseconds FWHM) is digitized by a peak sensing analog to digital converter (LRS-2259 $A D C)$. The peak voltage of the Gaussian pulse is directly proportional to the number of photodetached elec* cons. The ADC provides 10 bit resolution with maximum voltage of negative two volts corresponding to a binary count of 1024. A special $500 \mathrm{ohm} \times 5$ attenuator is required to drop the maximum preamp output of 10 volts (corresponding to 100 electrons) to 2 volts. An active inverter provides a negative polarity.

The ADC strobe is a 200 nanosecond pulse corresponding in time with the peak of the preamp output Gaussian pulse for photodetached counts. This stroba is generated by discriminator D3. A long ADC delay of approximately 2 microseconds is required to compensate for the slow risetime of the SSD pulse and achieve this timing. The triggering pulse is either the VPD trigger for coicidence events or the background trigger for background events. 
The fast ion chamber current monitor signal is also digitized by this peak sensing $A D C$. The time response of the fast ion chamber circuitry is comparable to the SSD channel. This gives digitization of beam current during the time window in which photodetachment occurred.

The particle counifing coincidence circuit for the SSD requires some cleverness. Timing accuracy of 50 ranoseconds is required to minimize false coincidences due to background. This is extremely difficult given the 5 microsecond wide signal pulses. A standard level crossing discriminator is unsatisfactory with the 100 to 1 range in signal amplitudes. A level crossing discriminator would translate even slight amplitude variations into large timing errors.

To avoid this problem, a single channel analyzer (SCA) is employed. This gives an output pulse which corresponds in time to the peak of the Gaussian input. The Ortec 427 SCA is adjusted to give an output spike which follows the occurrence of the peak of the input wave form by exactly 2 microseconds. By careful attention to the time walk adjustments, a timing jitter of less than 20 nanoseconds is obtained with a dynamic range of 100 to 1 . The amplitude window is set to accept pulses between 100 millivolts and 10 volts.

Discriminator D1 produces a narrow timing pulse which corresponds with delayed detector counts. These pulses 
are counted in visible and computer scalers designated ungated electrons. Ungated electrons give an indication of severity of gas stripping and allow indirect calculation of chamber pressure. The narrow pulses from D1 are ccmbined with a 100 nanosecond coincidence window to provide the coincidence output. This window is generated from either VPD triggers for real events or accidental triggers for accidental events. The triggers are delayed by approximately 4 microseconds to compensate for both SSD risetime and SCA delay.

The coincidence pulses are displayed on visible and computer scalers as described in figure 23. The scaler sums are quite useful for checkout and alignment. These scaler sums are also more accurate than $A D C$ sums in regions of very low cross section or when low laser power is used.

The oscilloscope and pulse height analyzer (PHA) provide convenient diagnostics and allow operation independent of computer controi. The delay amplifier is required so that the oscilloscope may be triggered from coincidence pulses to observe real events only.

\section{J. Timing Circuits}

Figure 25 is a timing diagram showing the relative timing of several important pulses occurring in the data acquisition system. Beam gates and $\mathrm{T}_{\mathrm{o}}$ pulses are received 


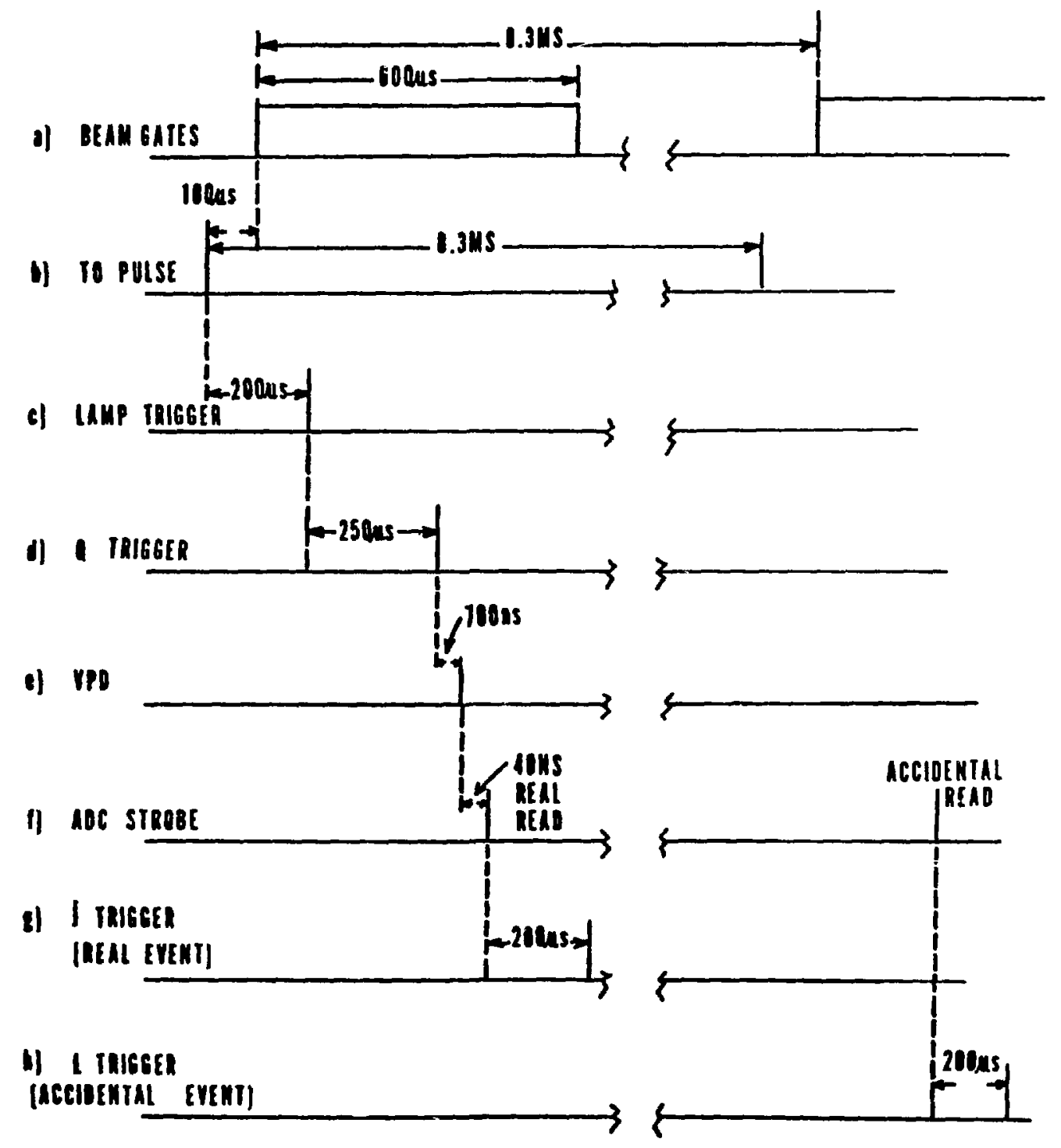

Figure 25. Timing Diagram 
from the accelerator control room. All experiment timing signals are derived from these signals.

The beam gates are high whenever beain current is present. If the beam current is momentarily shut off, the beam pulses are absent. The 600 microsecond width of the beam pulses corresponds with the current macropulse. The spacing between beam pulses is approximately 8.3 milliseconds corresponding with the 120 hertz beam current pulse repetition frequency. Many of the logic circuits are gated with beam gates insuring that data acquisition only occurs when current is present.

The beam current pulse train often displays missing macropulses. This occurs when selected macropulses are removed from our beam line and diverted to another experimental area. When this occurs, the corresponding beam gate pulses are deleted in the accelerator control room. If this occurs the laser trigger generator does not trigger the laser. In this case of missing current pulses, the laser is triggered on the first goud wacropulse after the beam current is restored to normal.

The $\mathrm{T}_{\mathrm{O}}$ pulses precede the beam pulse leading edge by approximately 100 microseconds. These pulses allow initiation of events before the current macropulse begins.

The laser trigger generator occupies one full NIM bin (Nuclear Instrument Manufacturer's standard equipment 
module). This complex unit provides mester timing signals for the data acquisition system as well as the lasei triggers. These are synchronized with the beam gates and $\mathrm{T}_{\mathrm{O}}$ pulses.

The laser trigger generator divides the basic 120 Hertz pulse repetition frequency (PRF) of the beam by 12 to produce laser triggers at a PRF of 10 Hertz. As discussed in the description of the laser, the flashlamp trigger must precede the Q-switch trigger by approximately 250 microseconds. This allows the YAG rod to be fully pumped before the Q-switch is fired. This delay is generated and controlled by the laser trigger generator. The laser Q-switch fires near the center of the macropulse. The lamps fire approximately 250 microseconds earlier.

The laser flash is monitored by the vacuum photodiode (VPD). This signal generates a fast trigger for strobing $A D C^{\prime} s$ and coincidence gating. The VPD trigger is the primary timing siginal and is slaved to the laser flash. The discriminator which generates the VPD trigger is located in the data conditioning fast electronics module and is gated with beam gates. Data acquisition thus occurs only during current macropulses. The VPD trigger occurs approximately 700 nanoseconds after the Q-suitch trigger. This delay results from the laser firing delay as well as the two way cable delay to the experimental area and back. 
In order to acquire background data, the VPD trigger is simulated on the next good beam pulse following the one in which the laser fired. The laser does not receive triggers in this pulse. Since the laser does not flash, this macropulse gives background conditions. The ADC and scaler channels are read in the background event. These data are subtracted from the coincidence event data to obtain real events.

The background or accidental trigger is generated in the laser trigger generator. This trigger is beam gated so that accidental read event cannot occur if beam current is absent. Instead, the background read will occur on the next good beam pulse.

The signal which tells the computer to read scalers and ADC's is generated in the laser trigger generator. These signals are termed " $j$ " and " $l$ " triggers for real and background events respectively. The triggers cause computer $j$ and 1 events via the CAMAC trigger module. The $j$ and 1 events cause the computer to read selected scalers. This occurs for each laser flash and accidental event. A similar trigger (k trigger) occurs at the end of each angle. The $k$ event causes the reading of the angle encoder to be recorded and signals a new set of data. Other triggers signal end of run, and start, or stop the run . 
Figure 26 is a simplified logic diagram of the laser trigger generator. Only essential logic units are displayed. These units are NIM (Nuclear Instrument Manufacturers) standard logic units. Fan outs and level converters are omitted for simplicity. All delays and gates of less than 1 microsecond duration are LeCroy LRS-621 discriminators. Delays of 1 microsecond or more are LeCroy LRS-222 variable delay generators. All logic gates are LeCroy LRS -364 fourfold logic gates.

The fundamental timing signal is the $T_{0}$ pulse. This pulse is delayed by D1 (see Figure 26) to fire the flash lamps at the desired time with respect to the beam current macropulse. This delay is adjusted to center the laser flash (VPD trigger) in the beam macropulse. The trigger out of Dl passes through AND gate Al firing discriminator D3. This is amplified to 10 volts by the line driver and sent to the laser as flash lamp trigger. This pulse also sets the 96 millisecond holdoff gate D2. This holdoff prevents the passage of the next 11 trigger pulses through AND gate Al. This achieves the division of the $T_{0}$ pulse rate by 12 to fire the lamps at 10 Hertz. Beam gates also form one input to Al. This prevents firing the lamps when beam current is absent.

The 250 microsecond delay of the Q-switch trigger with respect to flashlamp trigger is established by Qswitch delay $D 4$. This delay is adjusted to obtein maximum 


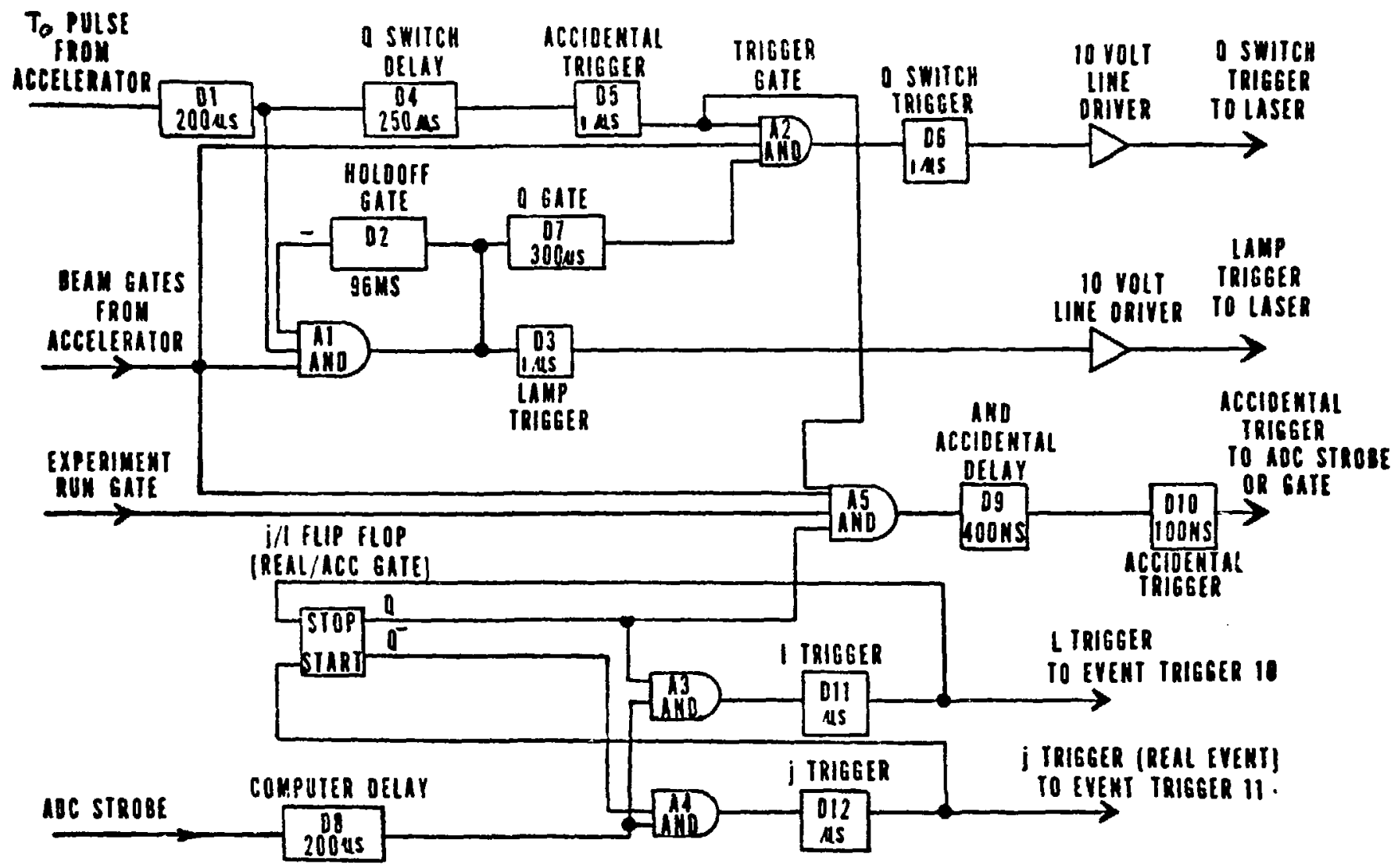

ALI GATES.LRS 364

DELAYS LESS THAH MS.LRS 621

DELAYS GREATER THAN AS - LRS 222

Figure 26. Laser Trigger Generator Logic Diagram 
laser power which occurs when the Q-switch fires at the point of optimum pumping.

The pulse from D4 triggers D5. This pulse passes through AND gate A2 triggering D6. The trigger from D6 is amplified to a 10 volt level and sent to the laser to trigger the Q-switch. AND gate A2 is held open by Q-gate D7 for 400 microseconds following each flash lamp trigger. Only during this period may the Q-trigger occur. This insures that a single Q-trigger will occur and that it will occur in the same macropulse as the lamp trigger which precedes it.

The pulse from D5 is also used to generate the accidental trigger which simulates the VPD pulse in strobing $A D C$ 's and forming coincidence windows for the accidental read (1 event).

The pulse from D5 after passing through A5, triggers accidental delay D9. Delay D9 is adjusted so that the accidental trigger occurs at the same time in the macropulse as the VPD trigger. A5 only allows the accidental trigger to pass during the macropulse following the laser flash. This is accomplished by the $j-1$ flip flop signal. The flip flop (actually a "jk" type of toggling flip flop) changes state approximately 200 microseconds after each laser flash. This arms the circuit by making the $Q$ output true. The following accidental trigger is passed by A5. 
200 microseconds later the flip flop changes state again, returning the circuit to its unarmed condition. The $Q$ output is true during the accidental read period only and false otherwise. The flip flop toggling is triggered by a delayed ADC strobe pulse from the data conditioning fast electronics module.

The $Q$ output of the flip flop is used to gate delayed ADC strobe pulses to either $j$ or 1 trigger outputs for commanding the computer to read scalers and $A D C$ 's for real or accidental events respectively. The gating occurs in and gates $\mathrm{A} 3$ and $\mathrm{A} 4$. The $\mathrm{ADC}$ strobe is delayed 200 microseconds in D8 to allow time for all scalers to stabilize before they are read. The same $Q$ and $\bar{Q}$ outputs are used to gate coincidences to real and accidental visible scalers through a pair of AND gates in the coincidence circuits. These have been previously described (see $A_{2}$ and $A_{3}$ in figure 23).

K. Computer Units

The operation of the experiment is controlled by the LAMPF standard data acquisition software system called Q. This is accomplished through a Digital Electronics Corporation PDP $11 / 34$ computer with Dataram 256 kilobyte memory and Plessy dual disk drive.

The experiment is actually self-operating with the computer monitoring and recording data as it flows in. 
The computer reads scalers and $A D C$ 's, starts the run, and can stop it as well. Otherwise the experiment free runs. Increment of angle is initiated by a trigger from the charge measuring comparator in the LAMPF Gate Generator. The computer is informed of angle changing by $k$ events but does not initiate the process.

Figure 27 shows a simplified block diagram of the computer hardware employed. The PDP-11 is the heart of the system. This unit, operating under the $Q$ code, directs the reading of scalers and $A D C$ 's after each $j$ or 1 event is received. The scaler sums are recorded in a disk file after each angle. A complete scan of angles is referred to as a "run". At the end of each run, a complete set of sums for each angle exists. This is called the dump file. Also recorded on each dump file are calibration parameters such as slopes and pedestals for each ADC, angle encoder offset and calibration, laser energy, and various other quantities required for the cross section calculation.

From time to time the disk files are copied onto a magnetic tape for permanent storage. These dump files are labeled by run number. Each scan is numbered sequentially. They contain entries on an angle by angle basis. Raw data are also recorded. These data are useful in later analysis. A run may be recreated at a later time 


\section{EXPERIMENT INTERFACE COHTROL OUTPUT}

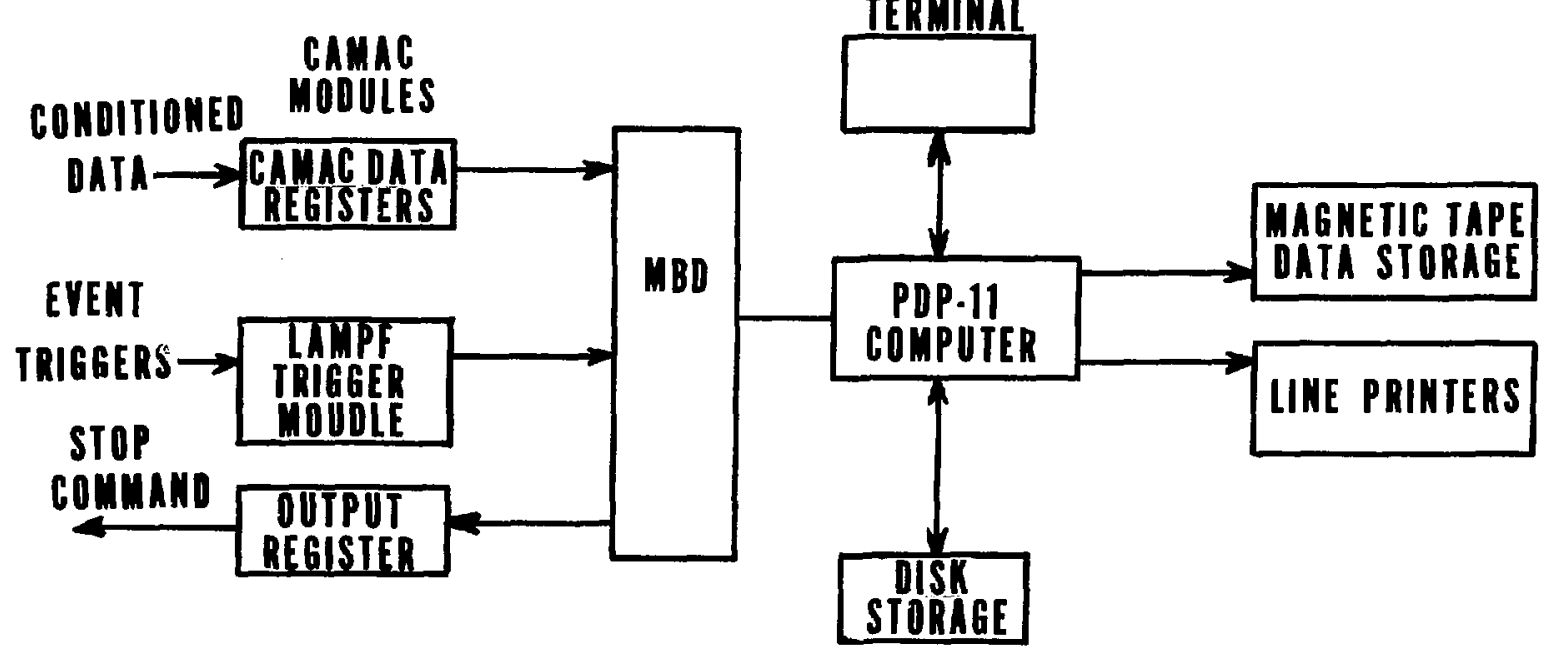

Figure 27. Computer Control Block Di.egram 
time using the raw data with a code called REPLAY. This allows pulse height analysis ( $\mathrm{PHA}$ ) and other procedures which must be done on a shot by shot basis to be accomplished in the analysis phase. The tapes are recorded by two Kennedy tape drives. Data are recorded in low density format ( $800 \mathrm{bpi})$ for both dump files and raw data files.

A Digital Electronics Corporation Decwriter terminal and Tektronics 4010 graphics terminal allow manual entry of commands and calibration parameters into the system. The graphics terminal provides online generation of histrograms while the run is in progress. Pulse height analysis provides a convenient diagnostic. This is quite useful for setting gains and attenuation to optimize use of limited $A D C$ dynamic range. The pulse height analysis is used to set ADC slopes and pedestals. This process is described in the discussion of calibration procedures. The data analysis code may also be run while data acquisition is in progress. This allows displays of calculated cross section as a function of angle.

A Printronix high speed line printer allows printout of scaler sums and cross sections. At the end of each run (a scan of angles) the line printer automatically prints an angle by angle list of photon energy and calculated cross section. The scaler sums and calibration parameters 
are also printed. If desired additional information may be printed. The line printer also allows a permanent record of any graphic display from the Tektronics 4010 to be recorded.

The PDP-11 does not read scalers and ADC's directly. A microprocessor-based interface unit designated MBD (Microprogrammed Branch Driver) actually reads the CAMAC modules. The MBD converts QAL commands into the CAMAC commands. The MBD also serves as a data buffer, allowing more efficient use of the primary computer. The MBD is an integral part of the LAMPF standard data acquisition system.

L. Data Analysis Code

Data analysis is accomplished by a data analysis code NCS (New Cross Section) which was created by D. C. Clark and M. E. Hamm for the experiment. The code calculates a separate cross section for each particle detector using Equation 23.

We attempt only relative cross section measurements. The scale factors on $I$ and $J$ are absorbed by the constant of proportionality which is arbitrarily set to a value which gives convenient cross section scale. For each detector, $\mathrm{N}$ must, however, be caículated correctly in order that counting statistics may be determined. This is accomplished by the calibration parameter called "slope". Observation of discrete peaks in pulse height spectra due 
to multiple photodetachments allows one to set "slope" so that $\mathrm{N}$ will be correct. This is done for each of the three detectors. Fractional errors (due to counting statistics only) are then calculated as reciprocal square root of $\mathrm{N}$ for each of the three channels.

Three values of $\mathrm{N}$ with error bars are calculated (corresponding to the three detectors) for each angle. The other quantitles used in the cross section calculation are identical for all three detectors. That is, the quantity inside the bracket in equation 1 need only be calculated once per angle. The bracket is then multiplied by the number of counts for each of the three detectors to give the corresponding cross section.

Relative cross sections only are calculated due to incomplete knowledge of absolute photon flux and beam overlap geometry. We can, however, obtain an accurate measure of the ratio of any two cross sections since the discrete number of detected particles is accurate (this is described in detail in the following section). It does not matter that the scale factor for photon flux, current or overlap geometry is unknown. When one takes the ratio of two simultaneously measured cross sections these factors cancel out. In fact this ratio could be correctly obtained without even measuring photon flux or incident beam current. Errors in normalization due to nonlinear 
measurement of the fluctuating laser flux and beam current are thus eliminated.

This is especially useful with two electron photodetachment data. The ratio of proton detector $\left(\mathrm{H}^{+}\right.$signal) to neutral hydrogen detector ( $\mathrm{H}^{\circ}$ signal) gives the two electron cross section a normalization relative to the one electron cross section. The one electron cross section is fairly well known. The ratio is much smoother than the $\mathrm{H}^{+}$signal. This is due to the independance from poor normalization as the $\mathrm{H}^{-}$current and beam overlap region change with time. The ratio of $\mathrm{H}^{+}$cross section to $\mathrm{H}^{\circ}$ cross section is calculated and displayed as though it were a fourth cross section. The fractional error bars for this ratio are obtained by adding the fractional error in the $\mathrm{H}^{+}$and $\mathrm{H}^{\circ}$ cross sections in quadrature.

Additional cross sections are calculated for $\mathrm{e}^{-}, \mathrm{H}^{\circ}$, and $\mathrm{H}^{+}$by using scaler counts rather than ADC counts. These are primarily useful for diagnostics. These cross sections display a marked saturation in regions of high count rate due to the inability of the scalers to count more than one simultaneous photodetachment.

The data analysis code NCS also allows several runs over the same energy range to be added together on an angle by angle basis. This provides improved counting statistics. Adding several brief runs is superior to 
taking one long run. This is because systematic errors due to slow drift of the apparatus are smoothed out. This procedure of adding many runs together is most useful for the two electron threshold data where the counting rate is very low. Files which are generated by adding several compatible dump files are called "sum" files. These sum files have the same format as dump files but appear smoother due to better statistics.

The NCS code allows the calibration parameters to be changed in the analysis phase. This allows one to correct energy calibration and ADC slopes. The NCS output can be displayed graphically. Any one of the cross sections may be displayed as a function of angle number, or up to four such graphs may be displayed simultaneously. The energy scale may be slightly non-linear in this format since photon energy is not a linear function of angle.

NCS also generates output data files which may be used to computer generate graphs with linear energy scales and error bars. These same output files serve as the input for curve fitting routines. The automatic curve fitting capability proved highly useful in relating experimental data to theory.

M. Calibration Procedures

Calibration is required to establish the relationship between angle encoder reading and photon energy. This is 
accomplished by observing the encoder reading of the $n=2$ Feshbach resonance (a narrow resonance feature in the single electron photodetachment spectrum). The energy resolution of the apparatus is measured by observing the width of the Feshbach which is essentially a delta function relative to the experimental resolution. It is also necessary to calibrate the gain of the particle detector channels in order that the $A D C$ counts will correctly reflect the actual number of particles counted in each channel. This is accomplished by observing multiple photodetachments with the computer PHA histogram display.

The equations of interest in energy and resolution calibration are

$$
\begin{aligned}
& E=\gamma E_{L a b}(1+\beta \cos \alpha) \\
& \frac{d E}{d \alpha}=E_{L a b} \gamma \beta \sin \alpha \\
& \frac{d E}{d T}=\frac{E_{L a b}}{m c}\left(1+\beta{ }^{-1} \cos \alpha\right) \\
& \frac{d E}{d E_{L a b}}=\gamma(1+\beta \cos \alpha)
\end{aligned}
$$

where $E$ is the photon energy in the center-of-mass frame, $E_{\text {Lab }}$ is the photon energy in the laboratory frame, a is the angle between the ion and photon beams, defined so that $\alpha=0$ for head on collisions, and $T$ is the ion kineEic energy. B and $Y$ are the usual relativistic constants. 
Equations 24,25 , and 26 are obtained from 3 by partial differentiation.

The laboratory energy of the laser is known quite accurately. Measurements performed by the manufacturer show the vacuum wavelength to be 1.064368 micron (energy = $1.1649 \mathrm{eV})$. These measurements are from interferometric comparison to a potassium standard. temperature effects result in negligible energy drift. The $\mathrm{H}^{-}$ion beam energy and zero of angle are not known nearly so well. Accurate calibrations have shown that 552.71 encoder steps corresponds to one jegree of rotation (See Section A). To calibrate the energy scale we must now obtain encoder zero. This is the angle encoder reading which corresponds with an angle alpha of zero degrees. From the Doppler formula, (Equation 3) it is seen that there are two values of $\alpha$ which correspond to the same energy. These occur for equal angular displacements clockwise and counterclockwise from encoder zero. By scanning the narrow Feshbach resonance on both sides of the beam, we obtain two encoder readings. The midpoint between these two readings is our encoder zero setting. This number is entered into the calibration parameters and is used to calculate the angle from the encoder setting.

The photon energy corresponding to the Feshbach resonance has been calculated with high precision by Broad and Reinhardt (1976). Using the theoretical value together 
with the angle (from the angle encoder reading) we solve the Doppler equation for $B$ and $\gamma$. We do this by successive iteration or by Newton's method. Having obtained $\beta, \gamma$ and $T$, we enter these as calibration parameters. At this point the energy scale is accurately calibrated giving correct photon energy as a function of encoder reading.

This energy calibration procedure must be repeated frequently due to large drifts in $\mathrm{H}^{-}$ion beam energy centroid with time.

We have used $10.930 \mathrm{eV}$ for the theoretical value of the resonance using the infinite Rydberg to convert the theoretical value to $\mathrm{eV}$. It now appears that we should have used the reduced Rydberg giving a value of $10.925 \mathrm{eV}$ for the Feshbach. This would result in a slight shift in energy scale. This small shift is generally insignificant.

The method of calibration gives extremely accurate measurement of the kinetic energy of the $\mathrm{H}^{-}$beam. This is a quantity of great practical interest to designers of particle accelerators. Since the laser flash is very fast the beam energy measurement is time resolved with a timing accuracy of approximately 10 nanoseconds. We have used this method to make highly accurate time resolved beam energy measurements at LAMPF (Clark et al 1979).

The resolution of our apparatus is limited by 3 factors: angular divergence, ion beam kinetic energy 
spread, and laser line width. These correspond with equations 24,25 , and 26 respectively.

The energy line width of the laser is less than 0.2 millielectron volts. The angular divergence of the photon beam is $0.5 \mathrm{mr}$, and the angular divergence of ion beam is 0.2 mrad. The spread in kinetic energy of the $\mathrm{H}^{-}$ions is typically $0.8 \mathrm{MeV}$ but is often much greater than this value.

Inserting these values in equations 24,25 , and 26 shows that the energy spread near the Feshbach resonance is dominated by the ion beam energy spread. The ion beam energy spread may be obtained from the measured Feshbach width using Equation 25. Figure 28 shows a typical scan of the Feshbach. This value of kinetic energy spread together with values of angular divergence and laser line width are then substituted into equations 24,25 , and 26 for any energy of interest. The three values of energy spread thus obtained are then added in quadrature to obtain the instrumental energy resolution at the energy of interest. Generally the spread is dominated by the $\mathrm{H}^{-}$beam energy spread. The one exception is for data taken near the one electron threshold at approximately .75 $\mathrm{eV}$ photon energy. With the YAG laser fundamental this is near the Doppler free angle (where equation 2 reduces to zero). In this case resolution is dominated by the angular divergence term. 


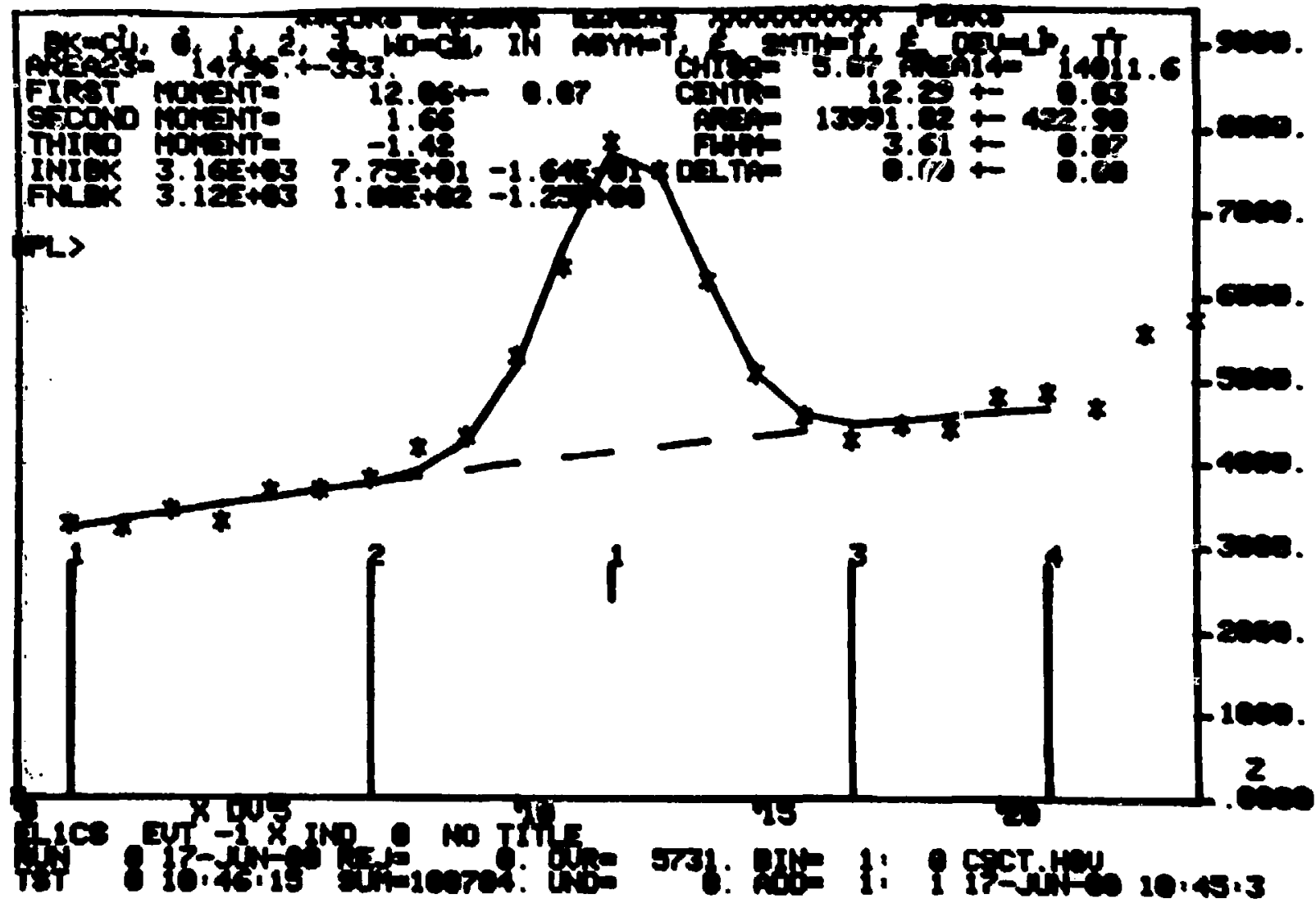

Figure 28. Feshbach Scan for Calibration 
In order to calculate statistical error bars, we must correctly count the number of photodetachments. Having the correct scale on the particle counters is also essential to obtain a meaningful normalization on the HPLHZE cross section.

Figure 29 shows a typical pulse height spectrum for a particle detector $\mathrm{ADC}$. Notice the multiple peaks corresponding to zero, one, two, etc., photodetachments. Markers are manually set to the center of the peaks. These correspond to pedestal level or zero photo detachments, one photodetachments, two photodetachments, etc. The first marker indicates the number of $\mathrm{ADC}$ counts corresponding to pedestal level. The average spacing of the markers corresponds to the number of $A D C$ counts per photodetachment event. These two numbers, called pedestal and slope respectively, are entered into calibration parameters. When this has been done for all three particle channels, the statistical error bars will be correct. Moreover the ratio of any two channels will be meaningful in an absolute manner. 


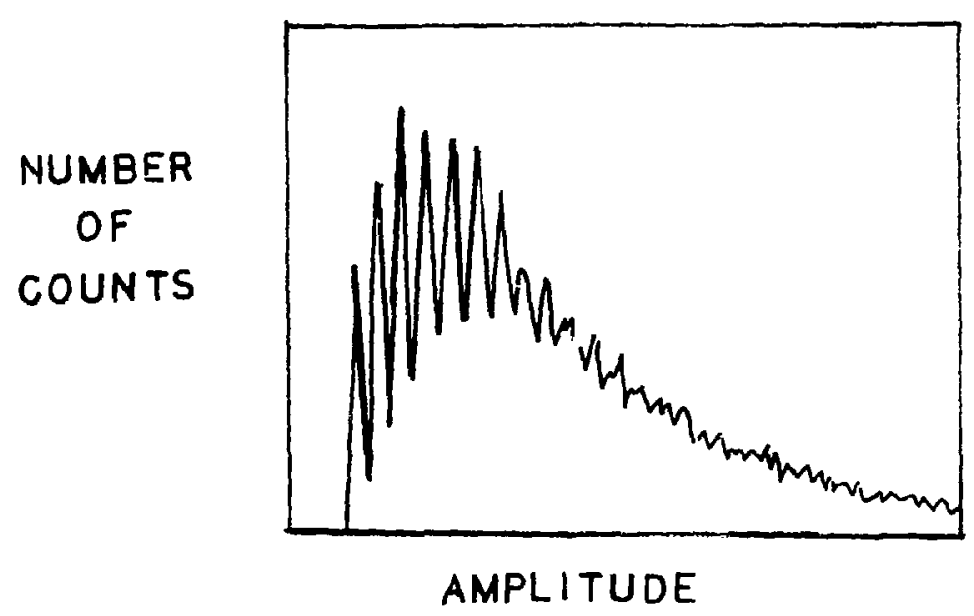

Figure 29. Pulse Height Spectrum From Particle Detector 
IV

RESULTS AND ANALYSIS

\section{A. Analysis Procedure}

The Doppler shifted laser technique has provided the first observation of two-electron photodetachment as well as the most precise measurements of the one-electron cross section and electron affinity to date. These measurements have been compared to theory with reasonable agreement. A few of the most significant results of this experiment have already been published (Bryant et al 1980 and Frost et al 1980). Additional results have been submitted for publication (Clark et al 1980 and Donahue 1980), and a review article is currently in preparation.

In the following sections the one-electron and twoelectron photodetachment cross section measurements are discussed. In each case the global scans showing general behavior and features are first discussed, followed by a section on the details of the threshold behavior.

The data are presented in the form of graphs, plotting the measured cross section as a function of effective photon energy. The cross sections are computed 
from counts in the electron, neutral hydrogen, or proton detectors (as discussed in the previous chapter). These are labeled $\mathrm{e}^{-}, \mathrm{H}^{\circ}$, and $\mathrm{H}^{+}$cross sections respectively and are presented with arbitrary vertical scale unless otherwise specified. The cross section ratio calculated by taking the ratio of $\mathrm{H}^{+}$signal to $\mathrm{H}^{\circ}$ signal has a meaningful vertical scale which is the actual ratio of counts in the two detectors. This signal is denoted by $\mathrm{H}^{+} / \mathrm{H}^{\mathrm{O}}$ " or the title "HPLHZE" on the figure.

In the case of the determination of the two-electron threshold law the statistical base is quite limited. The form of the law is found to agree with theory within the large uncertainty of the measurement. Having demonstrated the validity of the technique, we have been granted additional beam time in 1981 to obtain better statistics on this important measurement. It is hoped that the analysis techniques presented here will serve as a guide to analysis of this data which is to be collected in the future. When more than one scan over a given energy range is taken, these runs are generally summed (to smooth out random errors) as described in the previous chapters using the data analysis program NCS. Several runs may also be combined to obtain extended energy range. When runs are combined in this matter, the resulting sum files are stored on magnetic tape. When analysis is performed on 
oum files, the file name is given along with the result for future reference. For instance, the sum of all fine scans with 22 Gause deflecting fleld lo labeled '22F.SUM'. Thls flle name will be coneldered synonymous with the correeponding data.

The standard deviations on the graphs are computed from counting statiatice only and do not reflect other random and systematic errors in the data. In some cases the statiotical error bars are smaller than the points on the flgure and are not visible. For measurements of the two-electron threshold the statistical error bars are appropriate olnce the primary source of uncertainty is the ilmited counting atatistics. This is not true for most of the one-electron data where huge numbers of counts are easily obtained. In these measurements the statistical error is reduced to $a$ minute level and the uncertainity is dominated by other factors which are exceedingly difficult to quantify.

These sources of error Include random fluctuations due to imperfect normalization of the fluctuating beam current and overlap of the beams. There are also systematic errors due to misalignment of the optical system which can cause the overlap of the beams to change with angle in an unpredictable manner. This problem is referred to as 
"laser beam wander." Another cause of error is saturation or nonlinearity in the detectors. There are also slow drifts in calibration which do not effect the shape of the curve since each scan is taken rapidly. Slow drifts are thus not a great proble " for the relative measurements. The random fluctuations may be smoothed out by averaging a large number of measurements. The systematic errors are a much more severe limitations. Detectors were checked for linearity (when time permitted) by varying the laser power and beam current. The severity of laser beam wander may be checked by scanning the feature of interest for the equivalent Doppler angles on opposite sides of the ion beam. Disagreement of the two curves thus obtained indicate misalignment. None of these checks on accuracy was applied with any consistency due to limited time. Fortunately the errors due to laser beam wander are smallest near the threshold where the cross section changes rapidly with angle.

Attempts to quantify the non-statistical errors by comparison of repeated measurements were largely unsuccessful since 1) the systematic errors repeated on every measurement, 2) slow drifts caused large changes between repeated scans which were not true errors in that they did not effect the shape of the curves, 3) many of the measurements were not repeated. 
In comparing the experimental data points to theory we employ a curve fitting routine (MINUIT) to generate the 'best fitting' curve through the experimental data points. This is accomplished by varying the free parameters in the theoretical form so as to minimize Chi-square.

Chi-square is calculated from the formula

$$
x^{2}=\sum_{i} \frac{\left(\sigma_{1}^{t h}-\sigma_{i}^{\exp }\right)^{2}}{\Delta_{i}^{2}}
$$

where $\sigma_{i}^{\text {th }}$ and $\sigma_{i}^{\text {exp }}$ are the theoretical and experimental cross section values for the $i_{t h}$ data point and $\Delta_{i}$ is the experimental standard deviation for that point. In our case the statistical errors are used. This method is equivalent to a maximum likelihood calculation which gives the best possible estimate for the parameters.

The $x^{2}$ should be approximately one per degree of freedom where the number of degrees of freedom is the number of data points less the number of free parameters. When $x^{2}$ is significantly greater than one per degree of freedom we suspect that something is wrong with the theoretical form or with the data. It is clear from equation 27 that $x^{2}$ will be too large if the error bars are underestimated. This is generally the case with our data since the statistical error bars which we employ are always smaller than the true errors. The parameter values 
obtalned from fitting w1ll nevertheless be the beat estimates of the physical values. The confidence level may be obtained directly from the value of $x^{2}$ using tables. Such a table is used to calculate confidence level when it is quoted (Bevington 1969).

The curve fitting is accomplished by the use of a highly sophistlcated nonlinear optimization routine, MINUIT, (James and Roos 1975). This program has been under constant development at CERN since 1967 and 1s now a standard curve fitting procedure at the national accelerators. MINUIT uses a number of algorithms to minimize $x^{2}$ by variation of the free parameters in the most efficient manner. After a local minimum is obtained a Monte Carlo search can be employed to look for a better minimum. A detailed error analysis can also be performed using the covariance matrix to compute error bars and correlations. The computed error bars on a parameter correspond with the change in that parameter necessary to increase $x^{2}$ by one from its minimum value. This corresponds to one standard error for the parameter.

The off diagonal elements of the covariance matrix (error matrix) are used to compute the correlation coefficlents from

$$
\rho_{1 j}=v_{i j} / \sqrt{v_{11} v_{j j}}
$$


where $V$ 10 the covarlance matrix, $|P| \leq 1$ for al1 -lements. If $|e|=0$, the two parameters are unrelated. If $|P|=1$, the two parameters are completely correlated. The global correlation coefficlent for any parameter is the correlation between it and the linear combination of the other parameters most highly correlated with it. When high correlations are present, one could find a family of curves all fitting through the experimental points well but with widely ranging values for the parameter of interest. In this case $x^{2}$ might be very reasonable, Indicating a good fit, yet the parameter of Interest would be poorly determined. This type of hehavior is seen in the threshold laws, requiring extremely good data for an accurate determination of the exponent in a power law.

B. One-Electron Photodetachment

This section discusses several falrly coarse scans which together encompasa a photon energy range from .7 to $16 \mathrm{eV}$. Most of these scans were taken incidentally to other experiments. The scans were not repeated. Even considering the possible errors these are by far the best data over this important energy range. For this reason extensive analysis has been perforwed on the data. 
Figure 30 shows the one-electron cross section from $0.7 \mathrm{ev}$ to $2.7 \mathrm{eV}$. These data are the result of a single scan (RUN 1610) using the YAG fundamental wavelength with the deflecting magnets set at 380 Gauss. The cross section is obtained from the solid state electron detector. The relative cross section is plotted as a function of effective photon energy in eV. The features of interest are a sharp threshold at a photon energy of approximately $0.75 \mathrm{eV}$ and a peak near $1.5 \mathrm{eV}$.

The uncertainity in energy calibration of $5 \mathrm{meV}$ is much smaller than the 25 meV step size. Similarly the measured resolution of $1 \mathrm{meV}$, is much less than the step size.

The solid curve through the experimental points is the convenient parameterization of Armstrong (1963) normalized to fit the experimental data. The normalization is accomplished by using MINUIT to minimize Chi square. The plotted curve has the form of

$$
\sigma(E)=C+A \frac{E_{O}^{1 / 2}\left(E-E_{O}\right)^{3 / 2}}{E}\left[1+\frac{B_{O} E_{O}}{2}\left(2 E_{O}-E\right)\right]^{2}
$$

where $\sigma$ is the measured cross section, $E_{O}$ is the threshold energy in $\mathrm{eV}, \mathrm{E}$ is the photon energy in $\mathrm{eV}$, and $\mathrm{go}_{0}$ is an empirical shape parameter which is chosen for best fit.

This form is equivalent to Eq. 13. The scale factor A corrects for the arbitrary scale for our measurement. 


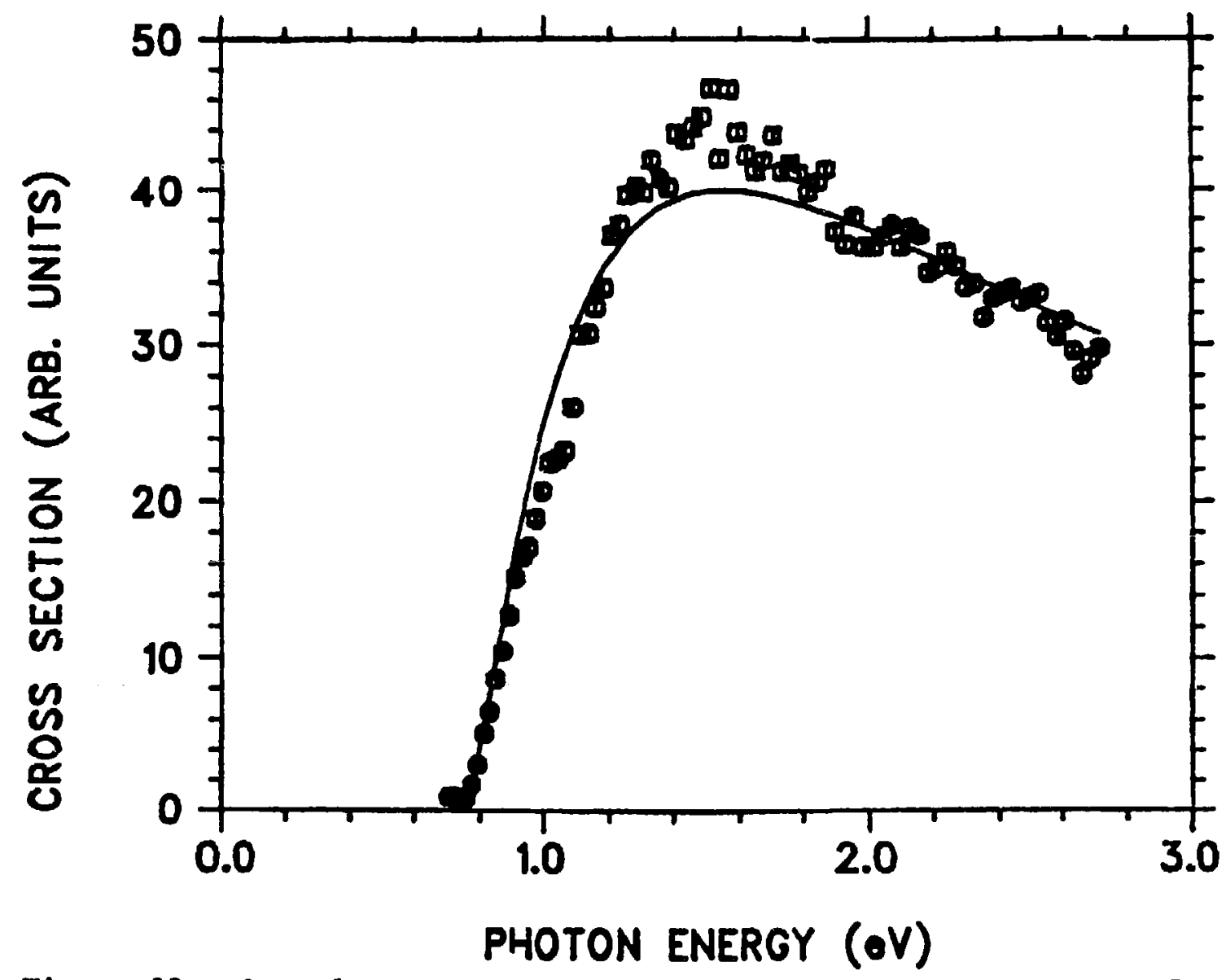

Figure 30. One-electron Cross Section Compared to the Theory of Armstrong 
while the beckground constant $C$ is required to correct for the small constant background below threshold. This background seems to be due to laser photons reflected back across the $\mathrm{H}^{-}$beam from the photon catcher. Even a slight reflection from the catcher would create algniflcant background, since the backward reflected photons have a Doppler shifted energy which is near the peak of the photodetachment curve.

$E_{0}$ was fixed at the theoretical value of $.7542 \mathrm{eV}$. The results of the fit are

$$
\begin{aligned}
& 80=-0.0611 \mathrm{eV}^{-2} \\
& \mathrm{~A}=135 \mathrm{eV} \\
& \mathrm{C}=0.203 \\
& \mathrm{x}^{2}=3059 \text { for } 77 \text { degrees of freedom } \\
& \text { The very large } \mathrm{x}^{2} \text { indicates considerable deviation }
\end{aligned}
$$
from the theory. This is seen by inspection of the figure. The theoretical curve rises more steeply from the threshold but folds over sooner than the data. The deviation is most pronounced from 0.9 to $1.6 \mathrm{eV}$. The statistical error bars are comparable to the size of the circles on the figure.

The high value of $x^{2}$ does not seem to be the result of Incorrect error bars. When the points below threshold were fitted by a constant, the resulting $x^{2}$ was only slightly over 1 per degree of freedom. 
The difference in shape could be an Inetrumental effect due to changing overlap of the baams as the splder rotates through large angles (leser beam wander). Th1s type of problem has been observed before and must always be coneldered when evalueting data covering a large angular range. Since the ecan was not repeatd, this matter cannot be definitely rasolved.

The shape is reflected in the value of the shape constant $80^{\circ}$ Armstrong (1963) finde a value of 4.7 when he flte his form to the date of Smith and Burch (1959). Th1e claselc data covered the energy range of $.9 \mathrm{eV}$ to 2.9eV. Our flt gives 8o $=-0.061$. By Inepection of f1gure 1 it 1s clear that the area of maximum disagreement 1s the low energy and of the curve. This ls also t.le region where Armetrong found greatest disagreement with the date of Smith and Burch.

It should be remembered that Armstrong's form, while physical in origin, is basically an explrical fit to the data where the factor so 1 e correction to make the model fit the data. Since our data give a much smaller correction, one suspects that the correction term is not required. Indeed, this would give a very simple parameterlzation of the one electron cross section,

$$
\sigma(E)=A \cdot{\frac{(E-E O)^{3 / 2}}{E^{3}}}^{3}
$$


Figure 31 shows the same data points (from run 1610) normalized to the theory of Broad and Reinhardt (1976). The solid curve is generated by a cubic spline fit through the theoretical values from table IV of the reference. These are reproduced in our table 2. The arbitrary amplitude scale factor and background constant are adjusted to fit the experimental points to theory. The energy scale is unaltered. This fit is no better than Arrstrong's simple parameterization yielding $x^{2}=9273$ for $\%$ degrees of freedom. Here too, the theoretical curve rises much more steeply from threshold and reaches peak at lower energy than the experimental data. This discrepancy may explain why Feldman (1970) and Weisner et al (1964) obtained incorrect values for the electron affinity of hydrogen.

An additional sian (run 1511) was taken in the energy range of 2.7 to $4.0 \mathrm{eV}$. These data did not fit the theory we11. Our group (Sharifian 1977) has previously taken data, using a nitrogen laser $(\lambda=3371 A)$, over the same energy region which fit the theory of Broad and Reinhardt much better. This indicates a possible problem with laser beam wander in the current data.

Figure 32 shows a scan (4K1.Sum) of $10.0-15.6 \mathrm{eV}$. This data was taken with the 3600 Gauss deflection magnet configuration. All 4 cross sections are displayed in raw 


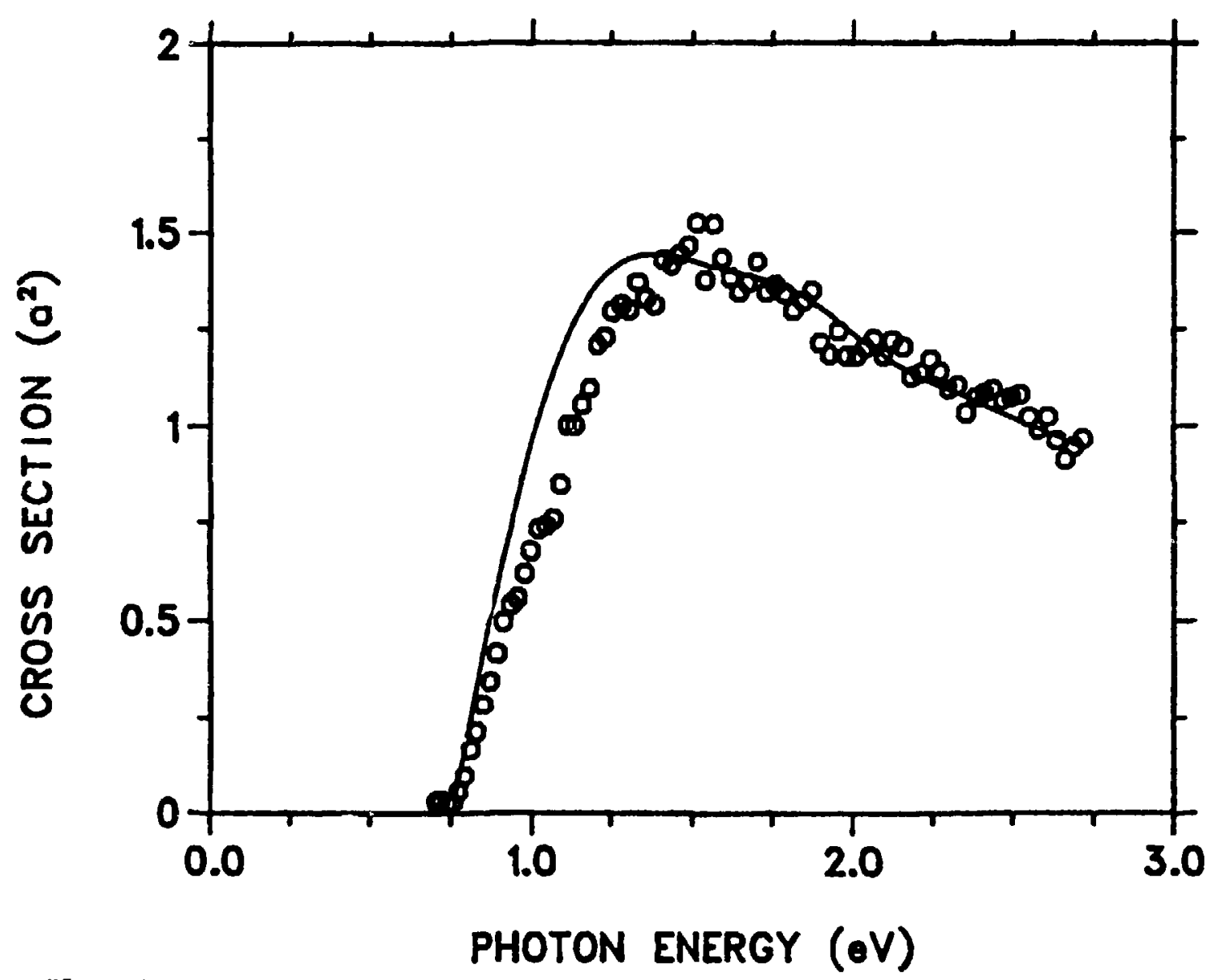

Figure 31. One-electron Cross Section Normalized to the Theory of Broad and Reinhardt E 

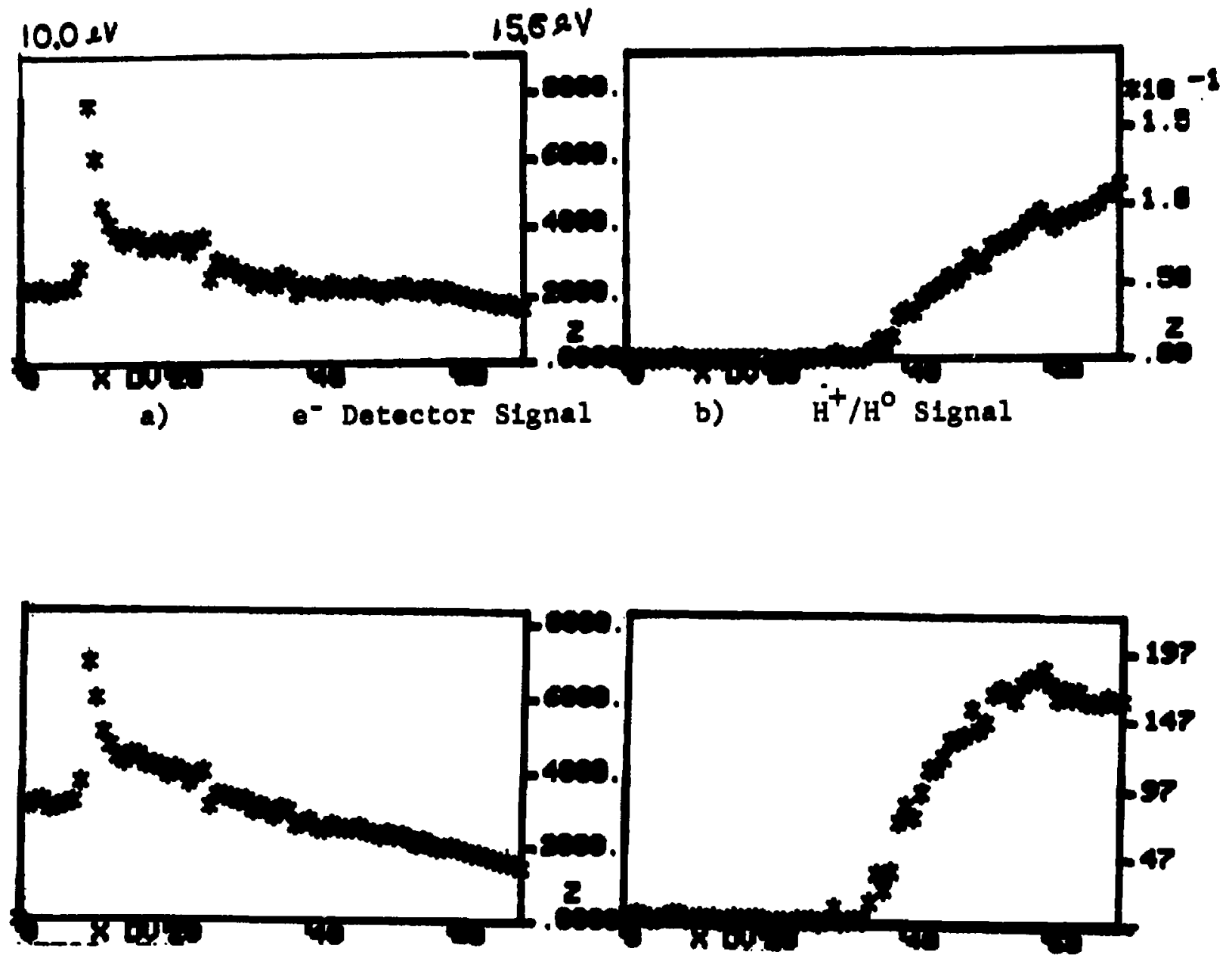

c) $\mathrm{H}^{\circ}$ Detector Signal d) $\mathrm{H}^{+}$Detector Signal

E1gure 32. Cross Sections from 10.0 to $15.6 \mathrm{eV}$ Raw Form 
form as they appeared during data acquisition. The cross sectlons are plotted an a function of angle number. They are as follows:

a. Cross section from the solid state electron detector

b. The ratio of $\mathrm{H}^{+}$to $\mathrm{H}^{\circ}$ counts

c. Single electron cross section from $\mathrm{H}^{\circ}$ sclntillator

d. Two-electron cross section from the $\mathrm{H}^{+}$ scintillator.

Not1ce the great accuracy with which the $e^{-}$and $\mathrm{H}^{\circ}$ channels track each other. Th1s Indicates that the total cross section to dominated by the aingle $e^{-}$cross section. Th1s agreement also Indicates correct setting of the ADC slopes (see callbration procedures) since the number of particles detected in each channel is identical.

The $\mathrm{H}^{+}$channel Indicates a threshold energy which is shifted down to approximately $13.5 \mathrm{eV}$ from 1ts theoretical value of $14.35 \mathrm{eV}$. This is the result of the large magnetic fleld which strips excited $\mathrm{H}^{\circ}{ }_{3}$. This phenomenon is described in section $C$. The data on two-electron photoejection are discussed in section $D$.

Figure 33 shows the cross section (from the $e^{-}$ detector) in more detail with resonance structure apparent. The $n=2$ shape resonance at $10.98 \mathrm{eV}$ (Bryant et al 1977) and as resonance dip associated with $n=3$ near 
5

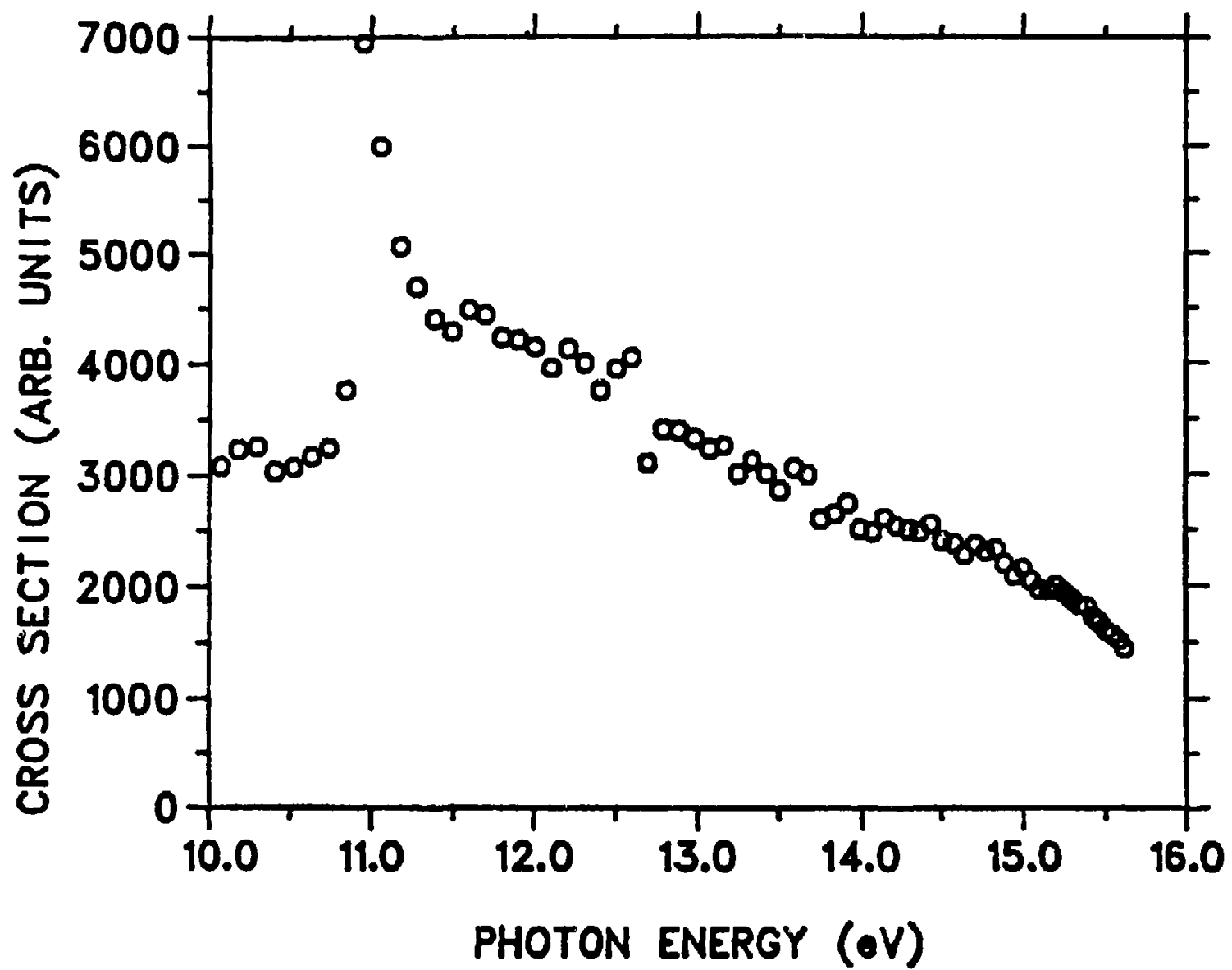

Figure 34. One-Electron Cross Section Coarse Scan (From $\mathrm{H}^{\circ}$ Detector) 
$13.65 \mathrm{eV}$ (Hamm et al 1979) are quite obvious. Figure 34 shows the one-electron cross section calculated from the $\mathrm{H}^{\mathrm{O}}$ detector.

These resonances are not the subject of this investigation. They do, however, provide convenient energy calibration markers. The improved apparatus makes study of the resonances much easier allowing considerable new resonance data to be collected in the course of the present experiment.

The resonances significantly affect the one electron cross section continuum. In figures 33 and 34 the continuum between $n=2$ and $n=3$ is seen to be lifted by the resonance structure. This adds considerable area to the curve. No resonance structure is visible for $n$ higher than 3 in this scan. There is, however, evidence in the field stripping data (See figure 43), to suggest the existence of resonances for higher $n$. These features are quite weak and totally masked by the continuum in the one electron data.

Notice the complete lack of any feature or discontinuity in the one electron cross section at the series limit $(14.353 \mathrm{eV})$. This may seem odd but it has been verified by repeated high resolution scans. The solid line is the theory of Armstrong fit to the data. This theory completely ignores the resonance structure and merely provides a convenient baseline. 


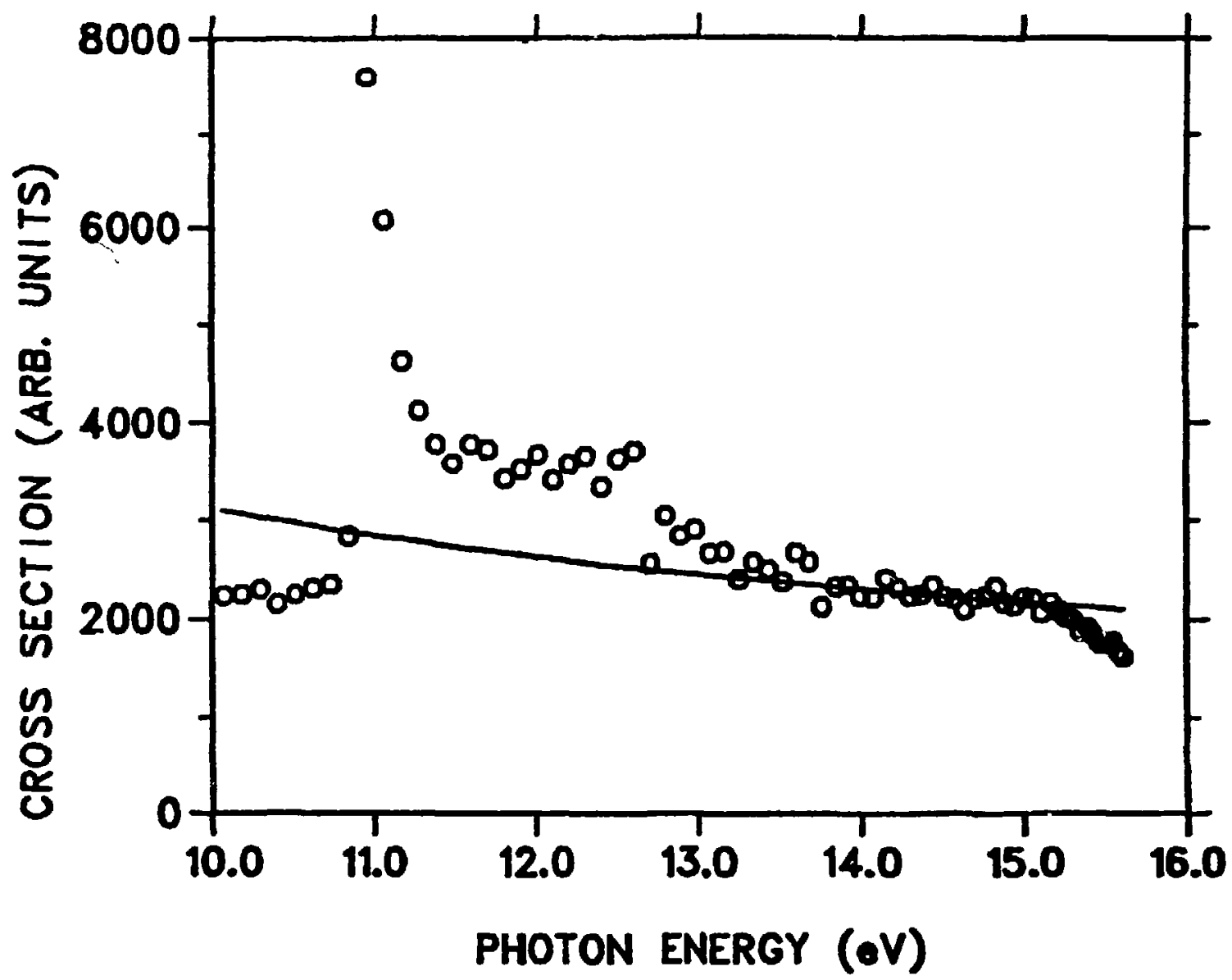

Figure 33. Cross Section From $e^{-}$Detector Coarse Scan 
A finer scan of the one electron cross section (from the $\mathrm{H}^{\circ}$ detector) over 13.5 to $15.5 \mathrm{eV}$ is shown in figure 35. The data (in units of square Bohrs) have been normalized to the theory of Broad and Reinhardt. These data (22C.sum) are the sum of a large number of scans added together. The 22 Gauss sweeping field configuration was employed to minimize field stripping. These data are of particular interest since they are used to normalize the two-electron cross section.

Close examination of figure 35 indicates a drop in the experimental cross section relative to the theory for the highest energies. The fall in cross section which becomes apparent above $15.2 \mathrm{eV}$ appears to be an instrumental effect due to nonlinearity on the photomultiplier tube associated with the $\mathrm{H}^{\circ}$ detector.

At the highest energies the laser beam and ion beam are almost collinear. The beams have a much larger intersection volume at small angles giving increased photodetachment rate. This increased rate is corrected for in the cross section calculation by the geometric factor $\frac{1+\beta \cos \alpha}{\sin \alpha}$ - If nonlinearity or saturation of the PMT occurs the calculated cross section will be depressed at high energies. The PMT is more susceptible to nonlinearity than the solid state electron detector (has limited 


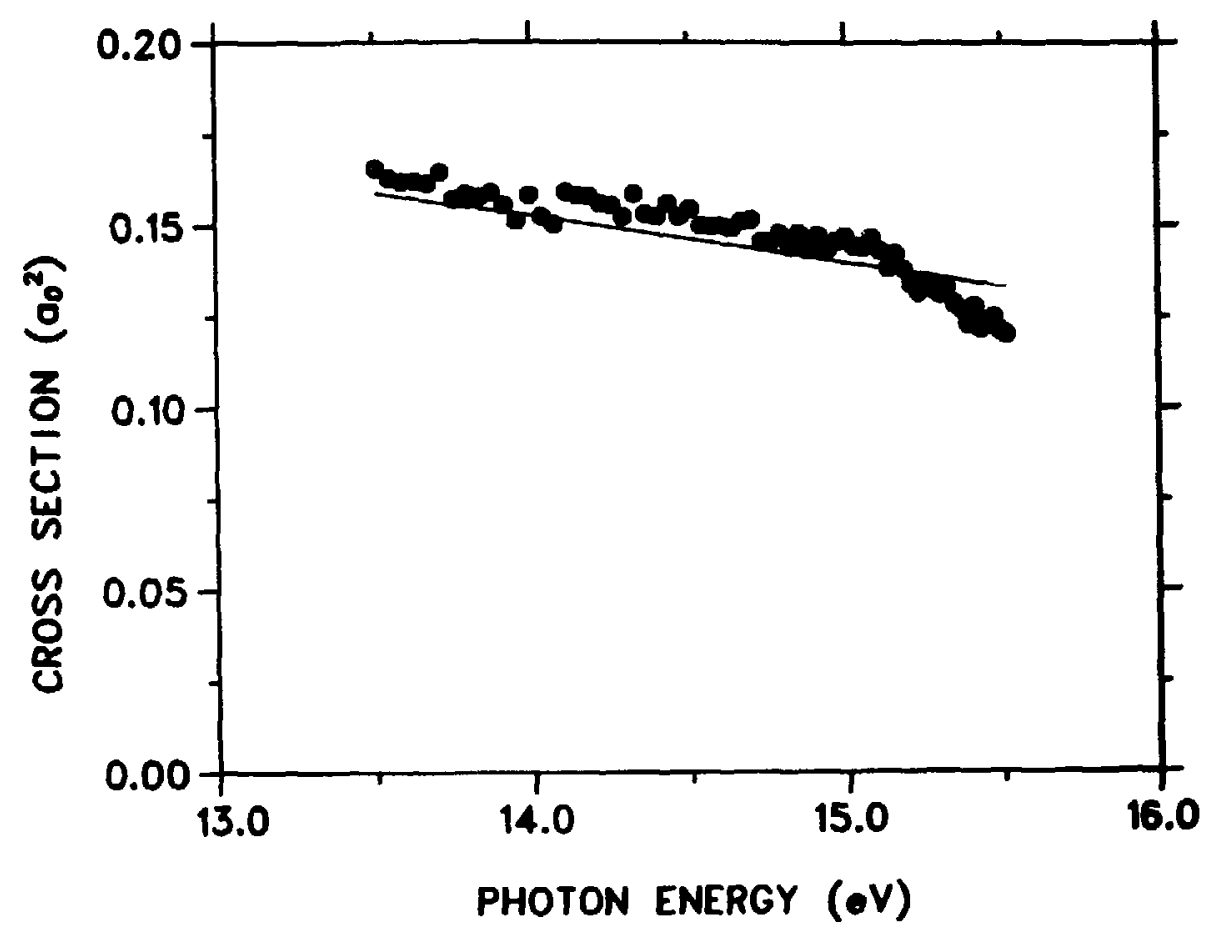

Figure 35. One-Electron Cross Section Normalized to Broad and Reinhardt 
dynamic range). We thus expect the effect to be more pronounced in the $\mathrm{H}^{\circ}$ channel than in the $\mathrm{e}^{-}$channel.

Close examination of figures 33 and 34 reveals a similar depression of the one-electron cross section at high energies. This scan with 3600 Gauss deflecting field was taken several months earlier when the laser beam alignment was completely different. The droop is much greater on the $\mathrm{H}^{\circ}$ channel than the $\mathrm{e}^{-}$channel. If beam wander were responsible, both $\mathrm{e}^{-}$and $\mathrm{H}^{\circ}$ channels would be equally depressed.

The ratio of the shape resonance peak to background is greater for the $\mathrm{e}^{-}$channel than for the $\mathrm{H}^{\mathrm{O}}$ channel. This also suggests that nonlinearity of the $H^{O}$ PMT is responsible for the high energy droop in cross section. A later diagnostic scan (RUN 2791) confirmed this nonlinearity in the $\mathrm{H}^{\circ}$ detector.

The droop does not seem to be a serious problem with the one electron data, giving at worst a $10 \%$ deviation from theory. It is, however, quite important in the analysis of the two-electron data which is normalized to the one-electron signal. Even a small discrepency can effect the shape of the normalized curve giving inaccuracy in the determination of the threshold power law. This is discussed in section $D$.

Figure 36 displays the one electron photodetachment cross section in units of square Bohrs from below 


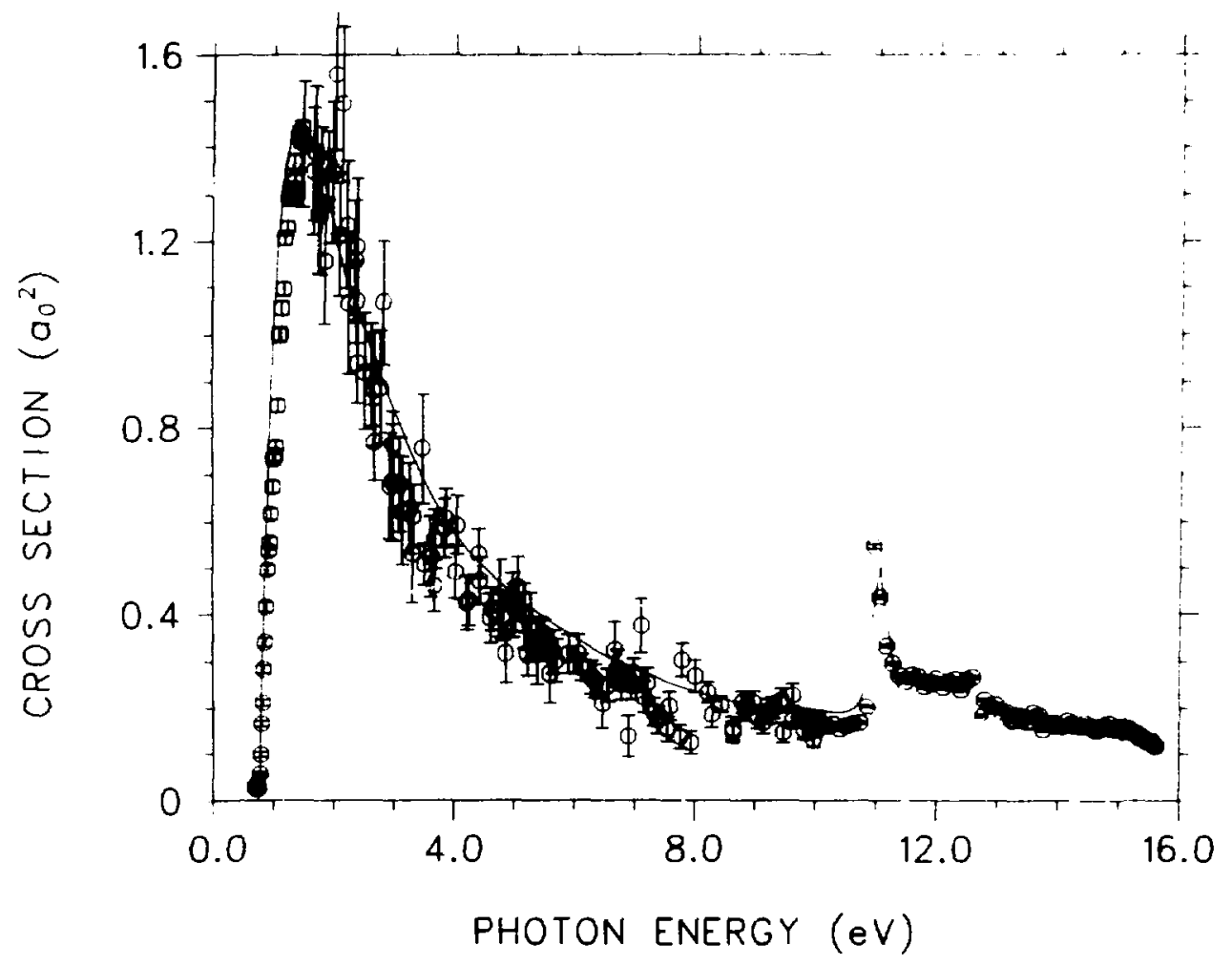

Figure 36. One-Electron Cross Section Global Scan 
threshold to $15.6 \mathrm{eV}$. The experimental data points are taken from run 1610 (figure 31), 4K1.sum (figure 33), and the nitrogen laser data (Sharifian 1977). The experimental points are normalized to the theory of Broad and Reinhardt (1976).

The solid curve is generated from the disprete theoretical points given in the reference by performing a cubic spline fit. In the $n=2$ resonance regicn points from figure 6 of the article are used. Outside this region points from Table IV (continuum photodetachment) of the same reference are used. These theoretical points are listed in Chapter 1 table 2 for convenience.

The three experimental curves are scaled in amplitude for best fit and combined to generate the continuous scan. The energy scales are unaltered. The agreement between theory and experiment is quite striking when viewed on this panoramic scale. The key features of this scan had been theoretically predicted yet only the broad peak at 1.5ev was observed experimentally previous to the relativistic beam measurements at LAMPF. This clearly illustrates the power of the technique.

C. One-Electron Threshold

The photodetachment threshold law for $\mathrm{H}^{-}$has not been investigated experimentally with high resolution previous to the present measurement. The electron affinity of 
hydrogen has been calculated with extreme accuracy (Pekeris 1962). Likewise, this value Eo $=0.754215 \pm$ $0.000002 \mathrm{eV}$ has not been challenged experimentally.

Having the opportunity to scan the threshold region with high resolution we have investigated the threshold power law, the energy range above threshold for which it is valid, and determined the electron affinity of hydrogen to high precision. Due to limited beam time and the fact that the one-electron data was not our primary mission, only 3 high resolution scans were taken over the interesting threshold region. Since only small angular range was scanned, laser beam wander should not be a problem with this measurement. The threshold data were detected using the solid state electron detector with the 380 Gauss sweeping magnet configuration. The fundamental YAG laser beam with a laboratory photon energy $E_{L}$ of $1.1649 \mathrm{eV}$ was employed. The threshold energy $E_{o}$ of approximately $0.75 \mathrm{eV}$ is obtained with an angle $\alpha$ of approximately 141 degrees. This is very near the Doppler free angle of 147 degrees (for $800 \mathrm{MeV} \mathrm{H}^{-}$beam). Typically the $800 \mathrm{keV}$ kinetic energy spread $\Delta \mathrm{T}$ of the $\mathrm{H}^{-}$ion beam limits our energy resolution, $\mathrm{dE}$, according to Equation 25. Near an angle of 141 degrees, $\mathrm{dE} / \mathrm{dT}=0.1 \mathrm{meV} / \mathrm{MeV}$. In this case the contribution due to beam energy spread is only $0.08 \mathrm{meV}$. Then for measurements near the one-electron 
threshold, the resolution is limited by the angular spread according to equation 24 . At $141^{\circ} \mathrm{dE} / \mathrm{d} \alpha=1.14 \mathrm{meV} / \mathrm{mrad}$. The sum of angular spread due to laser divergence and ion beam divergence is less than a milliradian. This provides resolution better than $1 \mathrm{meV}$ near threshold.

Since the energy scale is not highly dependent on the ion beam kinetic energy, we expect high accuracy in energy measurement. It would only be necessary to have a good pair of East-West Feshbach scans or to measure the threshold itself on both sides of the beam in order to calibrate the angle encoder zero. Unfortunately, no suitable scan of a feature on both sides of the beam was taken as a calibration. The energy scale is accurate to within 5 meV based on calibration data from previous runs. The scale is probably more accurate but we cannot be sure of this.

In figure 37 the measured one electron cross section in arbitrary units is plotted as a function of photon energy in eV. This figure is the result of a single scan (Run 1609) over the threshold region extending to an energy of $93 \mathrm{meV}$ above threshold. The step size of 3.69 meV/step is large compared with the instrumental resolution. A clear threshold is observed near $0.75 \mathrm{eV}$ photon energy. The constant background is assumed to be due to laser photons reflected backwards from the catcher 


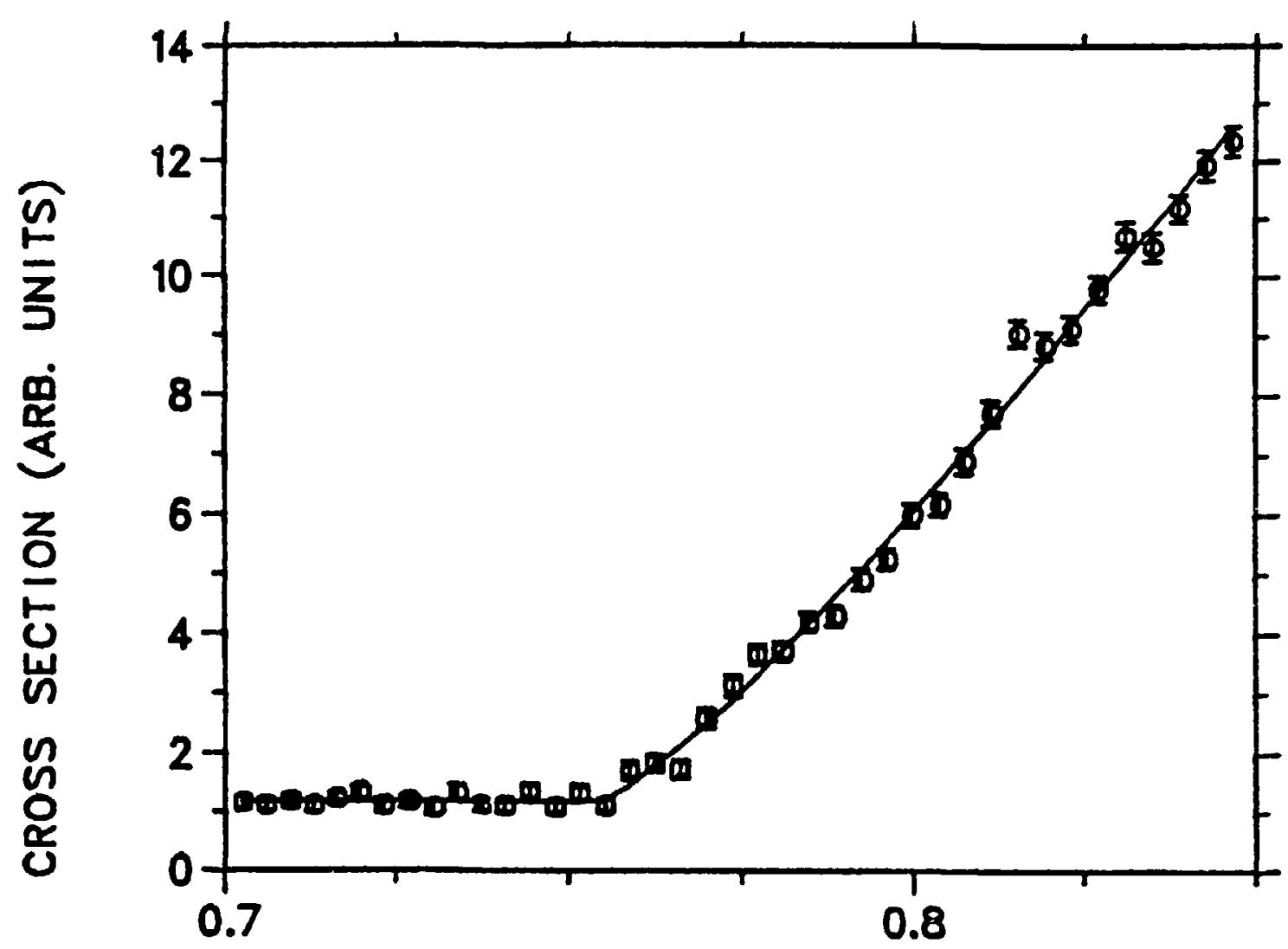

PHOTON ENERGY (oV)

Figure 37. Threshold for One-electron Photodetachment 
across the ion beam. This background is much more obvious here than in figure 30 since the signal is smaller very near the threshold.

The smooth curve through the data points is generated by fitting a power law with Minuit to minimize $x^{2}$. The form of the fit is

$$
\sigma= \begin{cases}C+A\left(E-E_{O}\right)^{N} & E>E_{O} \\ C & E \leq E_{O}\end{cases}
$$

where $\sigma$ is the measured cross section, $E$ the photon energy, $E_{0}$ the threshold energy, and $N$ the exponent which is 1.5 for the Wigner law. The scale factor A compensates for our arbitrary scale while $\mathrm{C}$ is required to give the constant background. $C$ is not part of the theory but rather due to instrumental background. Al1 parameters $\left(E_{O}, N, C, A\right)$ are allowed free. We wish to determine ' $N$ '. $E_{O}$ is free since it is determined more accurately by the curve fit than we could ocherwise set it given our uncertainty in energy scale. Results of the fit are

$$
\begin{aligned}
N & =1.207 \pm 0.029 \\
E_{O} & =0.755 \pm 0.001 \mathrm{eV} \\
A & =203.6 \pm 7.3 \\
C & =1.189 \pm 0.029 \\
x^{2} & =68.0 \text { for } 36 \text { degrees of freedom. }
\end{aligned}
$$

There are 36 degrees of freedom corresponding to 40 data points less 4 free parameters. The fact that the error 
bars in the data are statistical only may expiain the fact that the $x^{2}$ per degree of freedom is somewhat greater than one.

Inspection of figure 37 indicates a very close fit of the threshold law through the experimental points. The experimental value of $E_{0}$ given by the fit is within 1 meV of the theoretically predicted value. This 1 mel discrepency is certainly within the possible 5 meV uncertainty of our energy scale. The exponent $N$ is somewhat snaller than the theoretical value of 1.5 . This probably indicates a short range of validity for the $3 / 2$ power law. Since this scan contains points up to 93 net above threshold, one expects the exponent to be sonewhat less than $3 / 2$. The exponent must of course eventually fall to zero for high entrgy above threshold in order that the cross section may reach a peak. We employ a finer scan close to threshold to determine this exponent more accurately.

In figure 38 the threshold is scanned in smaller steps of $1.42 \mathrm{meV} / \mathrm{step}$. This data (Filename = lelth.Sum) is generated by adding two runs (1616 and 1617) over the same energy range which extends to $57 \mathrm{meV}$ above threshold. The amplitude scale is totally arbitrary and does not correspond with the actual data. The $5 \mathrm{meV}$ uncertainty in the energy scale corresponds to 3 data points while 


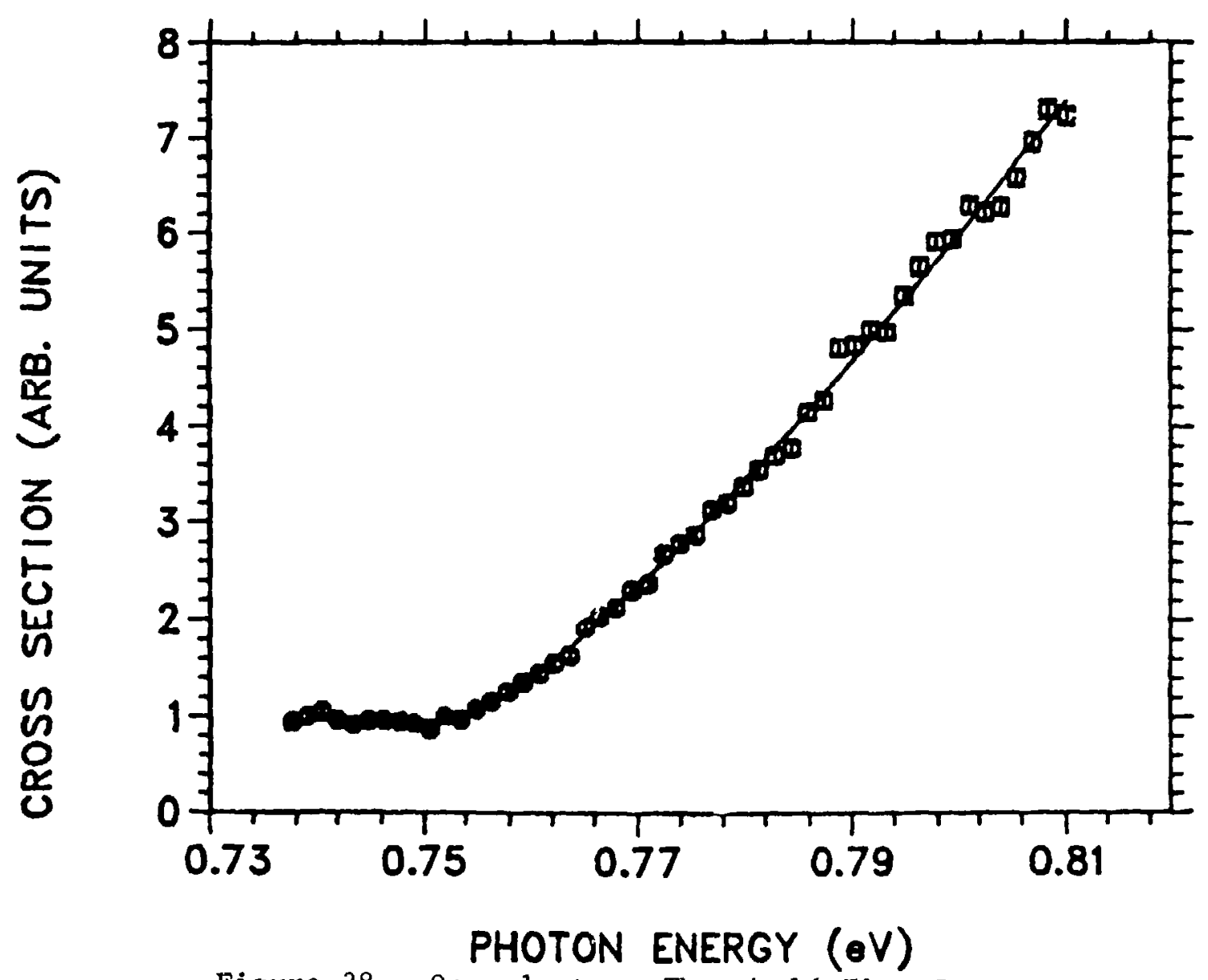

Figure 38. One-electron Threshold Fine Scan 
instrumental resolution of $1 \mathrm{meV}$ is finer than the step size. The constant background below threshold is assumed to be due to photons reflected from the catcher. The small oscillations appear to be due to imperfect normalization of the fluctuating beam current and overlap irtegral. These small non-statistical fluctuations are typical of our data and are not reflected in the calculated error bars.

As in figure 37 the solid curve is generated by fitting a power law using Equation 31 and allowing all 4 parameters to vary. The results are

$$
\begin{aligned}
\mathrm{N} & =1.279 \pm .026 \\
\mathrm{E}_{\mathrm{O}} & =0.7528 \pm 0.0005 \mathrm{eV} \\
\mathrm{A} & =799 \pm 10 \\
\mathrm{C} & =3.06 \pm .04 \\
\mathrm{x}^{2} & =99.1 \text { for } 46 \text { degrees of freedom }
\end{aligned}
$$

The large $x^{2}$ is probably due to use of statistical error bars in its calculation which ignores the nonstatistical fluctuations (See Sec. A). The experimental value for $E_{O}$ is again found to be within one millivolt of the theory. This agreement indicates that our $5 \mathrm{meV}$ estimate on energy scale uncertainty was probably quite conservative.

For this scan the exponent $\mathrm{N}$ is closer to the value of $3 / 2$. $3 / 2$ does not fall within the range of error 
bars. MINUIT generates the error bars by variation of the desired parameter so as to cause an increase in the $x^{2}$ of one from the minimum value. The other parameters are held fixed. This is done separately for each parameter.

In order to investigate the behavior of the function more closely, several separate fits were run each with a different fixed value of $N$. All other parameters were free. Figure 39 shows the resulting $x^{2}$ plotted as a function of $N$. It is clear from the figure that the function is well behaved and that the error bars on $\mathrm{N}$ which were calculated by MINUIT are correct. Forcing $N$ to $3 / 2$ gives a substantial increase in $x^{2}$ and is thus ruled out for this energy range of 57 mev above threshold. $\mathrm{N}=3 / 2$ may still be correct closer to threshold.

Also plotted on the same graph is $E_{O}$ (which was a free parameter) versus $N$. The value of $E_{O}$ thus obtained is roughly a linear function of $N$. For any reasonable $N$ (that is one which does not give great increase in $x^{2}$ ) the variation of $E_{0}$ from the value given by the fit is smaller than our energy uncertainty.

The fit of figure 38 is the most appropriate for determining $E_{O}$ since it uses all available data points taken at the smallest step size. Adding the error bars from fitting to the uncertainty in energy scale (in quadrature) gives the electron affinity of hydrogen as

$$
E_{0}=0.753 \pm 0.005 e V
$$




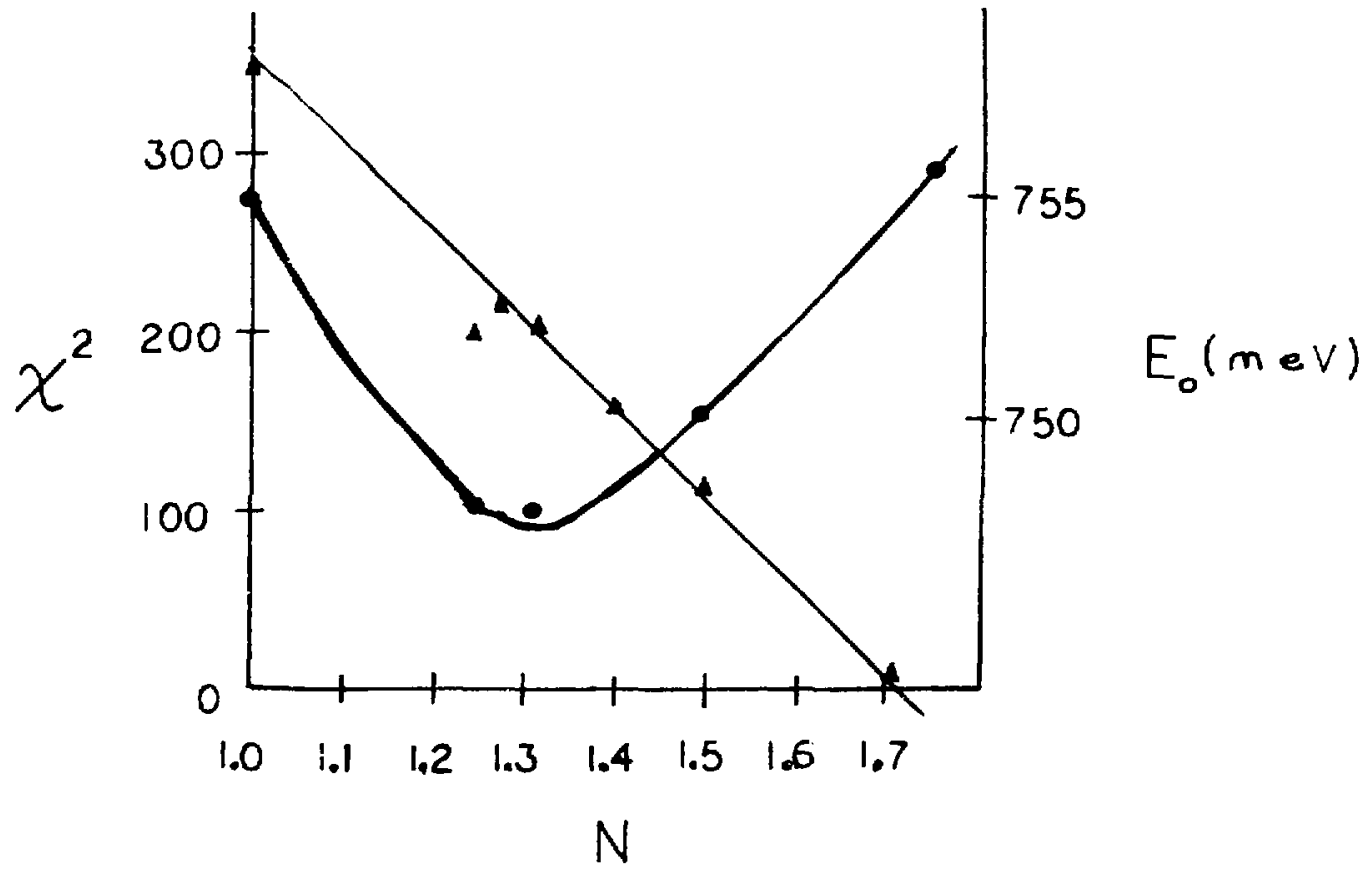

Figure 39. Plot of $x^{2}$ Versus $N$ 
This value is considerably more accurate than previous measurements and gives closer agreement with the theory. Table 5 shows various theoretical and experinental values for $E_{0}$. Our value agrees quite closely with Berry (1969). The reason for the over estimate in binding energy obtained by weisner and Armstrong (1964) enight be explained in terms of incorrect shape of the theoretical curve they employed. These investigators extrapolated data from higher energy back to the threshold. Figure 30 may show the problem with this. The theoretical curve, which is similar to the one used by Weisner and Armstrong, is seen to have steeper slope than the measured cruss section curve. Having only experimental points above $0.9 \mathrm{eV}$ extrapolation of the steeper slope to $\mathrm{E}_{0}$ would yield a high value for the electron affinity. Feldman's extrapolation used the $3 / 2$ power law over a range in excess of 100 meV. His result appears to suffer a similar shortcoming since the $3 / 2$ power law is no longer valid for points this far above threshold. Our method does not suffer from these problems since we have points within 2 meV of threshold.

In figure 40 only the first 25 points of the data from figure 38 are plotted. This gives energy range above threshold $(\Delta E)$ of $25 \mathrm{meV}$. 
Table 5. Energy of the ground state of $\mathrm{H}^{-}$. Number in parenthesis gives uncertainty in last digits (After Risley).

\begin{tabular}{|c|c|c|}
\hline$E(\mathrm{eV})$ & Method & Investigator \\
\hline \multicolumn{3}{|c|}{ Theoretical } \\
\hline$-0.754215(2)$ & Variational -444 order deter. & Pekeris (1962) \\
\hline$-0.754220(2)$ & Variational - 20 order deter. & Aashamar $(1970)$ \\
\hline \multicolumn{3}{|c|}{ Experimental } \\
\hline$-0.8(1)$ & Surface ionization & $\begin{array}{l}\text { Khoostenko and } \\
\text { Dukel' skii (1960) }\end{array}$ \\
\hline$-0.77(2)$ & $\begin{array}{l}\text { Extrapolation of photodetach- } \\
\text { ment cross section }\end{array}$ & $\begin{array}{l}\text { Weisner and Armstrong } \\
(1964)\end{array}$ \\
\hline$-0.756(13)$ & $\begin{array}{l}\text { Stellar photodetachment } \\
\text { spectrum }\end{array}$ & Berry $(1969)$ \\
\hline$-0.776(20)$ & $\begin{array}{l}\text { Crossed-beam, photodetach- } \\
\text { inent threshold }\end{array}$ & Feldman (1970) \\
\hline$-0.753(5)$ & $\begin{array}{l}\text { Crossed-Beam, photodetach- } \\
\text { ment threshold with Doppler } \\
\text { tuned laser source }\end{array}$ & This Experiment \\
\hline
\end{tabular}




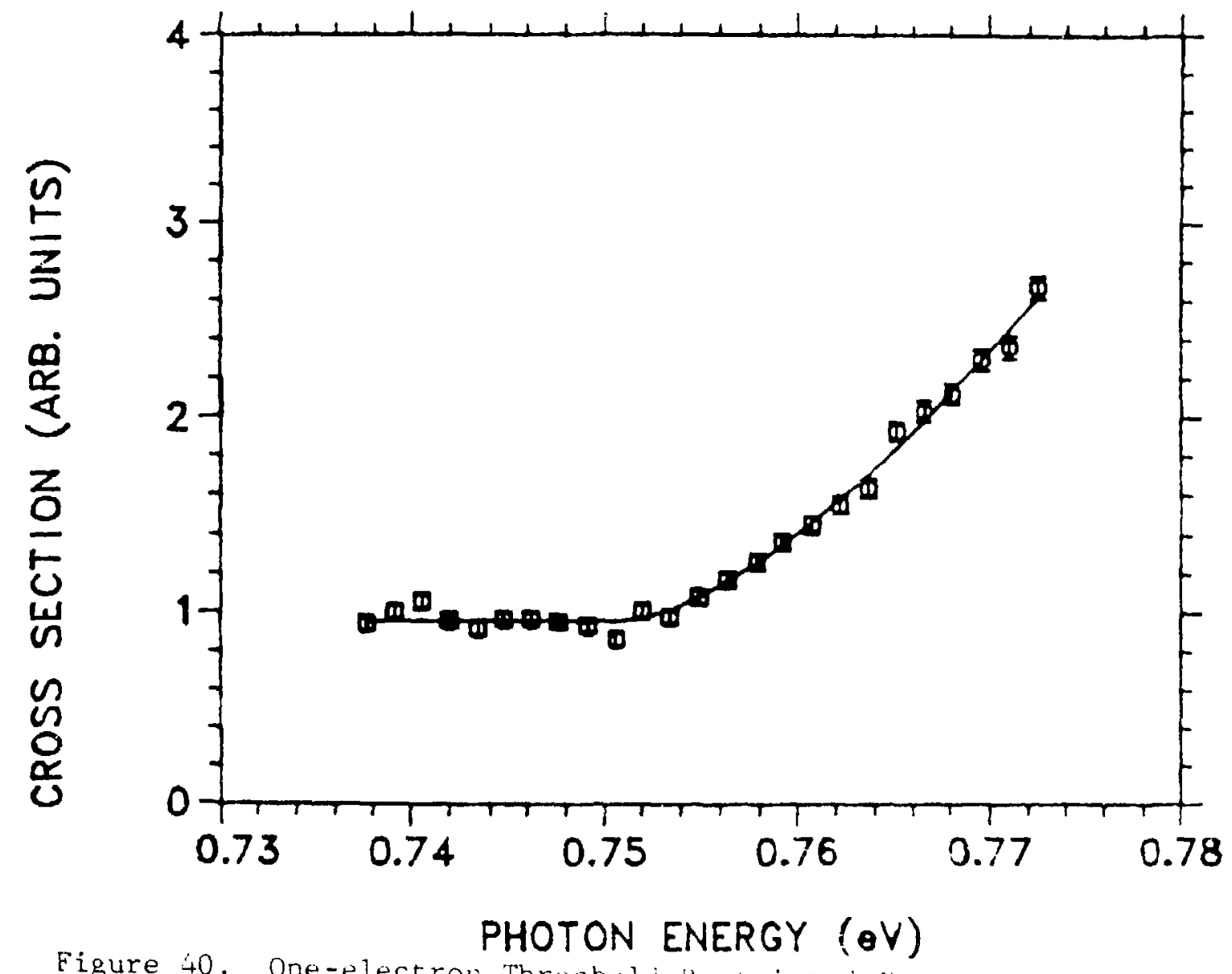

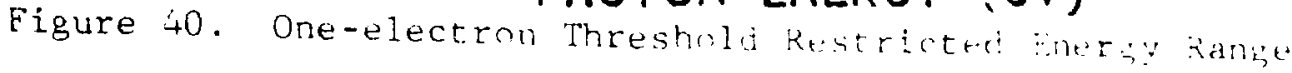


Fitting gives

$$
\begin{aligned}
N & =1.496 \pm .202 \\
E_{O} & =0.751 \pm 0.002 \mathrm{eV} \\
A & =1710 \pm 21 \\
C & =3.05 \pm 0.04 \\
x^{2} & =29.4 \text { for } 21 \text { degrees of freedom. }
\end{aligned}
$$

$E_{O}$ is consistent with the previous fits. $N$ is now in accord with the predicted $3 / 2$ power law within the limits of the larger error bars.

To further investigate dependence of $N$ on $\Delta E$ (the range above threshold) several fits were made over various energy ranges above threshold. The form of the fit is from Equation 31. Data from runs 1610, 1609, and (1616+ 1617 ) is fitted to give $\Delta E$ from $23 \mathrm{meV}$ to $600 \mathrm{meV}$. Knowing $N(\Delta E)$ and extrapolating to $\Delta E=0$ gives the threshold behavior. Figure 41 show $^{-}$the results of these fits with $\mathrm{N}$ plotted as a function of the range above threshold whicl was considered in the fit.

The plot clearly shows the decrease in exponent as the energy range is increased. This is the expected behavior. Extrapolation of the curve to zero energy is consistent with the Wigner threshold law, indicating an exponent of 1.4 to 1.7 would be reasonable.

Additional fits for an energy range of less than $23 \mathrm{meV}$ would be most desirable. Attempts were made to fit 


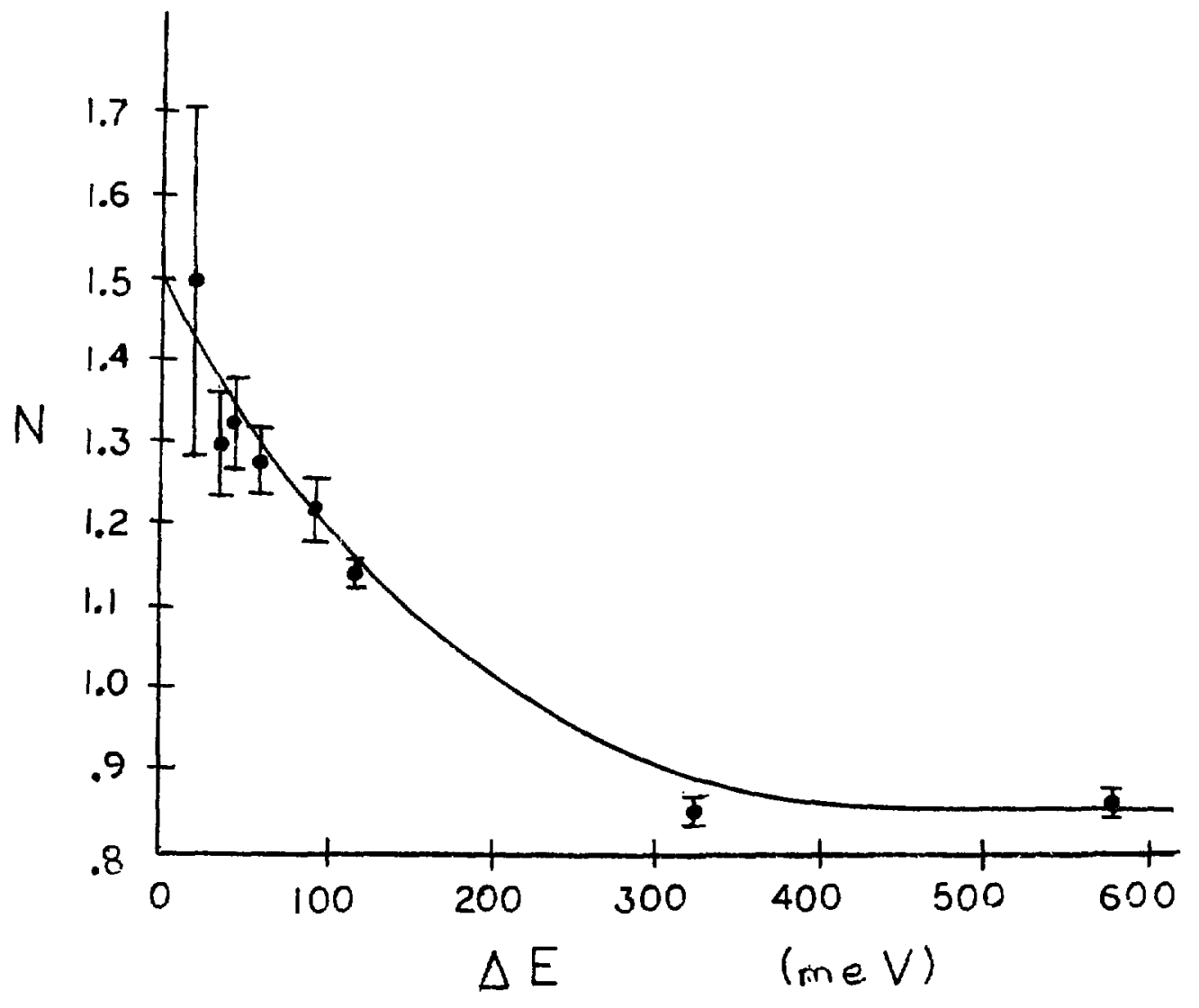

Figure 41. Plot of $N$ Versus Energy Range 
the data closer to threshold but this was found to be impossible. MINUIT, the curve fitting routine, would not properly converge for any value of $\Delta E$ less than 23 mev. This was due to the limited number of data points and poor statistics near threshold. The difficult functional form, which had extremely high correlation between the parameters $A$ and $N$, did not help.

The solid curve drawn through the points of figure 41 is merely one possible form of the function which defines: dependence of $\mathrm{N}$ on $\triangle E$. A theoretical description of this curve would be most helpful in extrapolating the available data points back to zero ralues. As discussed in chapter 2 , the $3 / 2$ power law is actually the leading term of a power series expansion. For finite energy range the higher terms become significant and describe the departure from the $3 / 2$ power law for increasing energy. These terms might be obtained from the data of figure 41, a?lowing improved extrapolation to zero energy range.

In summary, analysis of the three scans indicates consistency with the $3 / 2$ power law. Extrapolation to zero energy range gives the exponent $N$ somewhere between 1.4 and 1.7. The data of figure 41 indicates the range of the law and clearly displays the decrease of $\mathrm{N}$ with increasing range. The electron affinity of hydrogen has 
has been determined to be $0.753 \pm 0.005 \mathrm{eV}$ in agreement with the theoretical values of Pekeris (1962) and Aashamar (1970).

\section{Magnetic Field Stripping}

An unexpected background signal was observed in the initial phases of the two-electron photodetachment. experiment. The background which was very large and was energy dependent totally obscured the desired signal. Great efforts were made to reduce the background. In the final hours of the experiment this was accomplished, allowing the measurement to be successfully completed. The background is quite interesting in itself. It provides a way of viewing photodetachments into higher excited states and pllows observation of a single channel into a particular state. This opens the door to a whole series of new experiments to measure branching ratios. For this reason and because of its relevance to the twoelectron data, this background will be discussed in some detail.

The signature for the two-electron photoejection is a count in the $\mathrm{H}^{-t}$ detector. We use a magnetic field to separate the protons from the incident beam (see figure 16). Near the two-electron threshold large numbers of $\mathrm{H}^{\mathrm{O}}$ 's are produced in highly excited states by one-electron photodetachments. The highly excited $\mathrm{H}^{\mathrm{O}}$ 's can be field 
ionized (stripped) by the motional electric fleld from the separating magnet and then counted in the proton detector. These counts produce an energy dependent noise signal that can completely mask the desired two-electron photoejection signal. This process was discussed briefly in the previous chapter under 'magnet configurations'. The excited $\mathrm{H}^{\mathrm{O}}$ 's may also be ionized by collision with residual gas molecules (enhanced gas stripping), absorption of a second laser photon, or even by absorption of black body radiation from the environment (Gallagher 1978). In our case the first process (field stripping) dominates due to the large deflection field employed.

In summary we wish to observe the reaction

$$
\gamma+\mathrm{H}^{-}+\mathrm{H}^{+}+2 \mathrm{e}^{-} \text {. }
$$

The primary background is the two step ionization,

$$
\begin{gathered}
\gamma+\mathrm{H}^{-}+\mathrm{H}^{\circ}(\mathrm{n})+\mathrm{e}^{-} \\
\mathrm{H}^{\circ}(\mathrm{n})+\mathrm{field}+\mathrm{H}^{+}+\mathrm{e}^{-},
\end{gathered}
$$

where the second step occurs only for $n>n_{c} \cdot n_{c}$ is the lowest excited state ionized by the magnet and varies as the inverse fourth power of the deflection field. The minimum $B$ field required to ionize (for $B=0.842$ ) excited $\mathrm{H}^{\circ}$ is given approximately by $\mathrm{B} \cong \frac{68}{\mathrm{n}^{4}}$ where $\mathrm{B}$ is in Tesla (Bayfield, 1979). 
In the absence of the background, we would see a threshold for proton production at $14.35 \mathrm{eV}(13.60+.75)$. In reality we see a false threshold at an energy lower than $14.35 \mathrm{eV}$. The location of this anomalous threshold corresponds to the energy $E_{c}$ required to create $H^{\circ}\left(n_{c}\right)$ which is $.75 \mathrm{eV}$ to remove one electron from the ion plus the energy to raise $\mathrm{H}^{\circ}$ from the ground state to the state $\mathrm{n}_{\mathrm{c}}$. That is

$$
E_{c}=.754 \mathrm{eV}+i 3.598 \mathrm{eV} \frac{n_{c}{ }^{2}-1}{n_{c}{ }^{2}} .
$$

The onset energy of the anomalous threshold depends on the magnetic separating field. By reducing the separating field, the anomalous threshold may be brought very close to the true threshold. Due to the sparse population of the highest excited sigts, this also reduces the level of the noise signal to a very low value above threshold.

In practice the reductiun nf separating field may not be extended indefinitely. In our case the separating field was reduced to 22 Gauss which stripped only $\mathrm{H}^{\circ}$ states with $n \geq 14$. For our geometry further reduction gave insufficient separation of the photoejected beam from the primary beam. We could further reduce the field to perhaps 3 Gauss while increasing the length of the magnet to give the same $\int B d 1$. If the field were further reduced, extensive steps would be required to prevent the fluffy 
Rydberg states from being stripped by stray fields or even room temperature black body radiation from the environment.

In figure 32 we saw that the threshold was depressed to $13.50 \mathrm{eV}$ corresponding with $\mathrm{n}_{c}$ of 4 . This is consistent with the theory of field stripping for the 3600 Gauss field. The theory predicts that 2700 Gauss will strip $\mathrm{n} \geq 4$

Figure 42 shows the anomalous threshold associated with the 3600 Gauss deflecting field in more detail. The cross section is calculated from counts in the $\mathrm{H}^{+}$ detector. The vertical scale is arbitrary. This scan ( $4 \mathrm{~K} 4$.Sum) is the result of adding a number of runs over the same energy range. The structure below $13.81 \mathrm{eV}$ is totally tue to the field stripping of $\mathrm{H}^{\mathrm{O}} \mathrm{s}$ in the $\mathrm{n}=4$ state since photon energies below $13.81 \mathrm{eV}$ are insufficient to excite ions to $\mathrm{H}^{\mathrm{O}}$ in the $\mathrm{n}=5$ state while the electric field associated with the 3600 Gauss magnet is insufficient to ionize $\mathrm{H}^{\mathrm{O}}$ 's with $\mathrm{n}<3$. This region then gives a view into the single $\mathrm{n}=4$ channel $\left(\gamma+\mathrm{H}^{-}+\mathrm{H}^{\circ}(4)+\right.$ $e^{-}$) below the $n=5$ threshold.

At $13.81 \mathrm{eV}$ we see a clear step which is the $n=5$ threshold. The thresholds provide sharp energy calibration markers of precisely known energy. Since they are near the two-electron threshold, they may be used for 


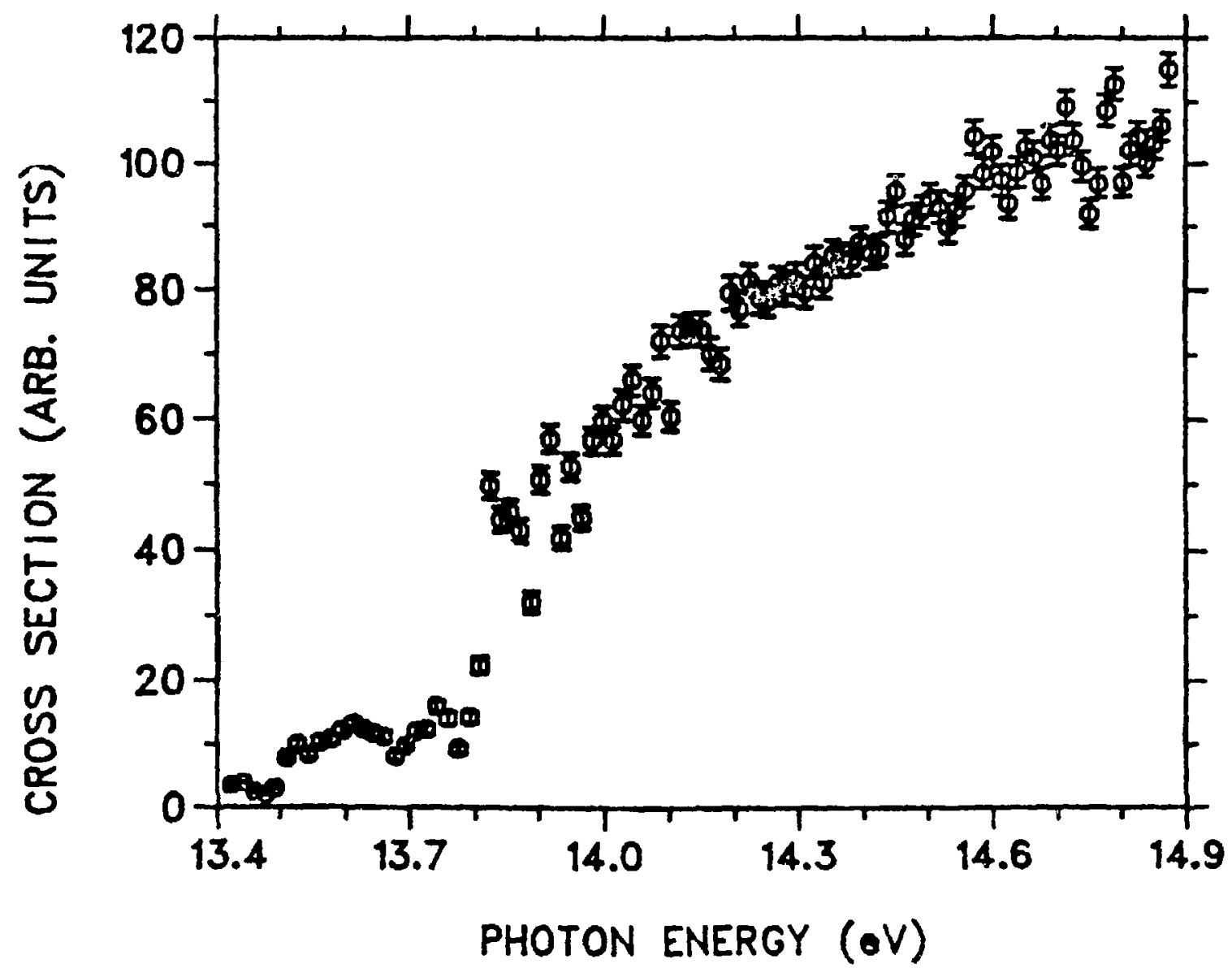

Figure 42. Cross Section From H+ Detector with a Stripping Field of 3600 Gauss 
energy calibration of two-electron data. Scanning a threshold on both sides of the lon beam gives encoder zero as well as beam energy calibration.

Above the $n=5$ threshold are a series of dips. These dips appear to be associated with resonances below the $n=6$ threshold and are presumably similar to the $n=3$ dips (Hamm et al 1979) observed in the one-electron cross section. The dips are completely obscured in the one-electron cross section due to the large continuum background. By selectively observing channels into $n=4$ and 5 , we can clearly see these structures. Figure 43 shows the structure in this region in finer detail. Recently a similar structure has been observed in the $\mathrm{He}^{+}(\mathrm{n}=2)$ cross section by photoionization of ground state He (Woodruff and Samson $1980)$.

In figure 42 the cross section continues to rise as additional $n$ states become excited. The continued rise in cross section above the series limit at $14.353 \mathrm{eV}$ is assumed to be due to the onset of two-electron photoejection. The two-electron cross section is riding on top of a very large background of unknown energy dependence. Extraction of the two-electron signal from such background is an exceedingly difficult problem. One approach to this problem is to understand the background more fully. The one-electron photodetachment 


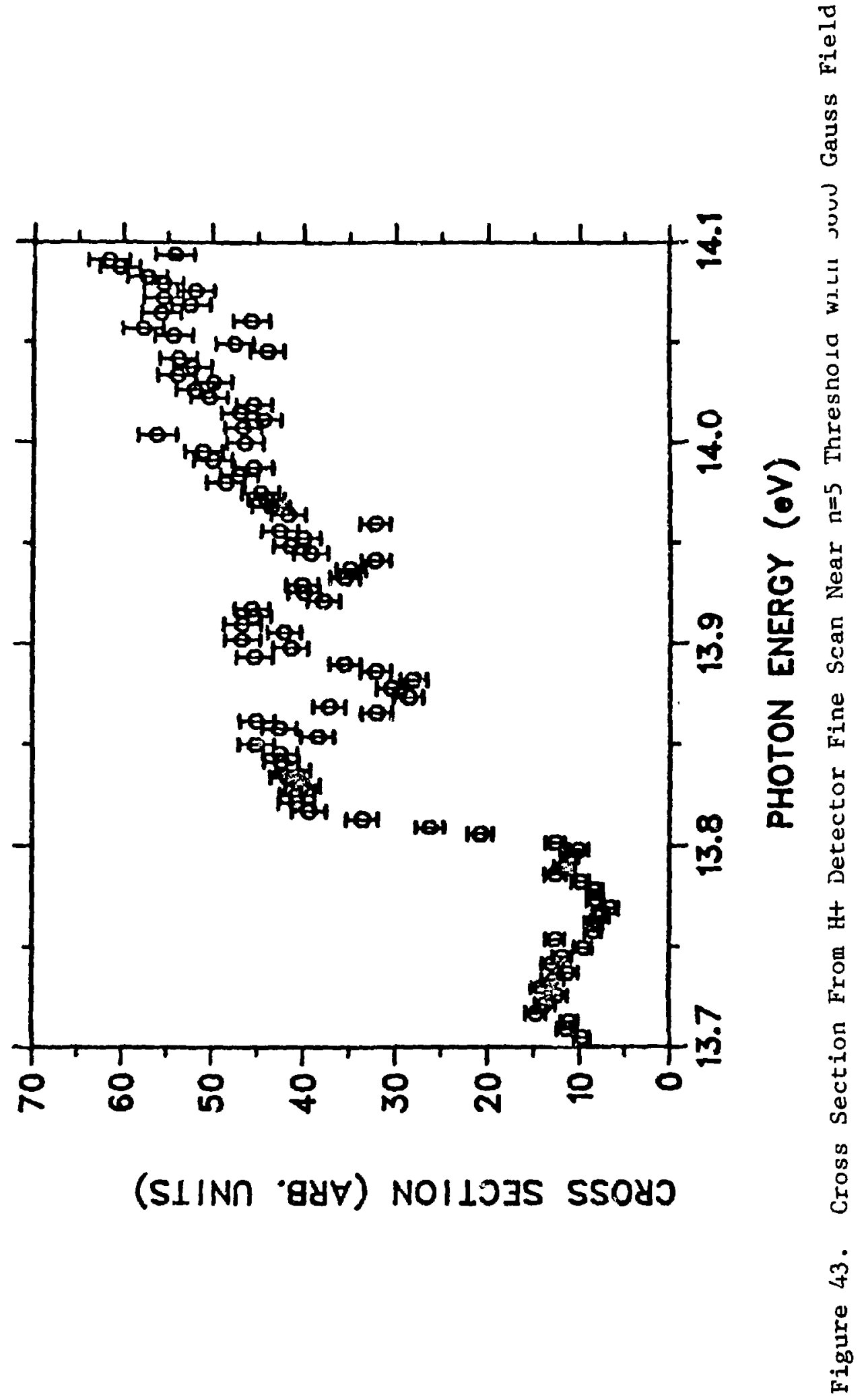


into the higher excited states near the threshold involves exactly the same quantum mechanics which determines the (wo-electron photoejection threshold behavior (Fano 1980). It has been suggested that the photodetachment into Rydberg states just below threshold may be viewed as a airror image of the process above, with the energy above threshold replaced by the energy below (Temkin 1980). The mirror imaging is not a straight forward reflection. Moreover, the presence of resonance structure will certainly complicate the picture, making interpretation of the measurement difficult. Nevertheless, efforts are currently underway in this direction as well as in further sludy of the resonance structure seen in the field stripped background.

A cleaner approach to the problem and one less prone to misinterpretation is simply to reduce the magnetic separating field to the point where the field ionized background may be ignored. We have proceeded in this direction. The initial field of 3600 Gauss (which strips $n>4$ ) was reduced in steps to 22 Gauss (which strips $n \geqslant 14$ ). This field reduced the background to acceptable levels. The reduction of deflection field was no easy task. In order to obtain separation of the $\mathrm{H}^{+}$from the primary beam a long flight path of 20 meters was required. The steering of the photoejected protons onto the $3 / 8$ inch 
scintillator was very difficult. Slight changes in ion beam angle of entrance to our apparatus resulted in varying collection efficiency on the detectors. For thit; reason the ratio of $\mathrm{H}^{+}$to $\mathrm{H}^{\circ}$ suffers an uncertainty of approximately a factor of 2 for the lowered stripping field measurements.

The effect of reducing the stripping field will be described in steps. Figure 44 shows the cross section calculated from $\mathrm{H}^{+}$detector with a stripping field of 2200 Gauss. This scan ( $2 \mathrm{~K} 1 . \mathrm{Sum}$ ) is the result of adding several runs over the same energy range. The scans looks much like figure 42 except that the signal due to stripping of $\mathrm{n}=4$ is absent. This agrees with the field ionization calculations which indicate 2700 Gauss would be required to strip $n=4$. The step at $n=5$ is clearly present as is the resonance structure below the $n=6$ threshold. This structure is seen in finer detail in figure 45 (2k2.Sum). This $\mathrm{H}^{+}$scan also employs a 2200 Gauss defiection field.

Further reducing the stripping field to 780 Gauss pushes the anamolous threshold up to $14.07 \mathrm{eV}$ corresponding with $n=7$. This is seen in figure 46 (780F.sum) which is a fine scan over the threshold region. The cross section from the $\mathrm{H}^{+}$detector is displayed with arbitrary vertical scale. The stairstep structure is 


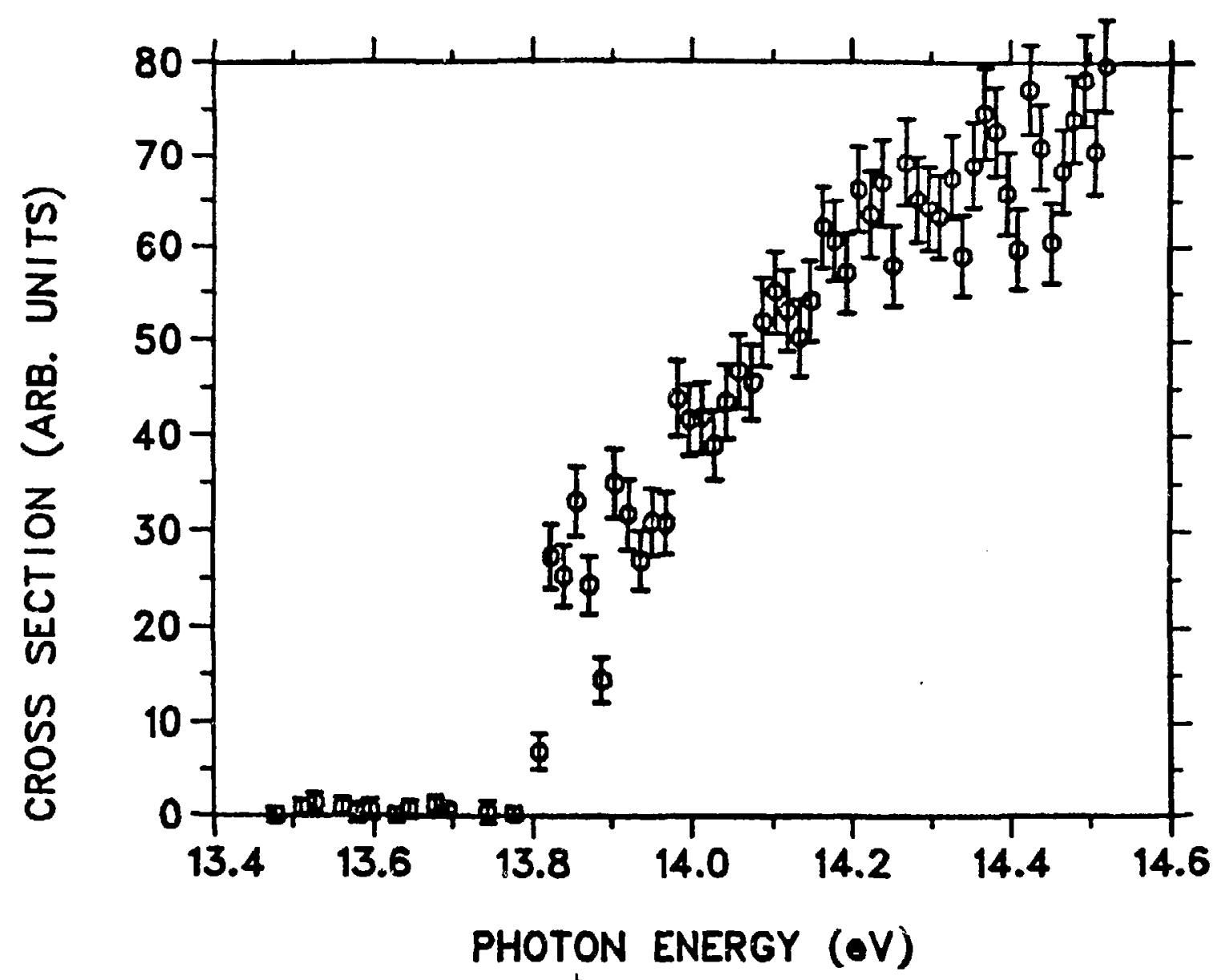

Figure 44. Cross Section From $\mathrm{H}^{+}$Detector with 2200 Gauss Field 


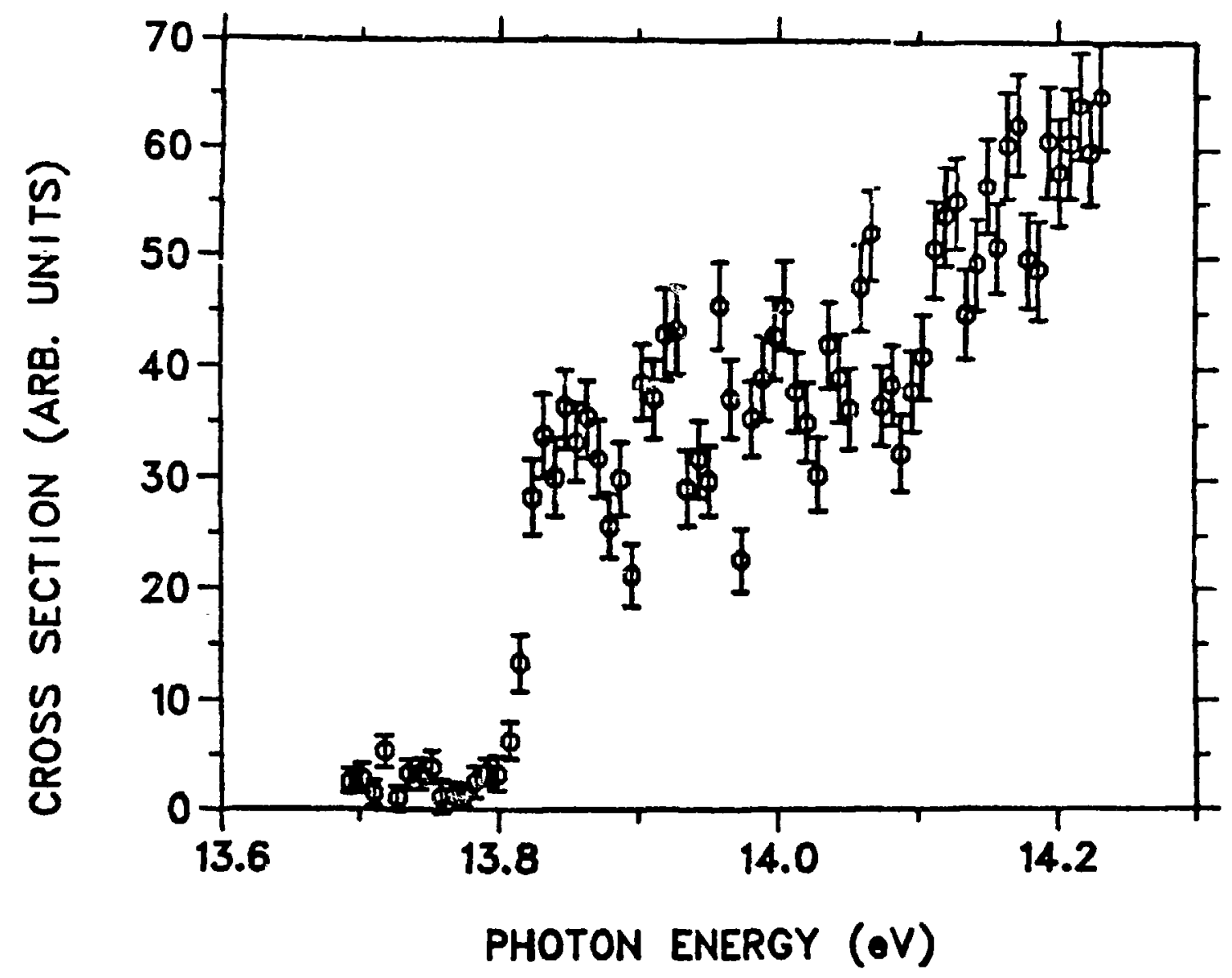

Figure 45. Fine Scan of Cross Section Calculated with $\mathrm{H}^{+}$Detector Signal (B $=2200$ Gauss) 


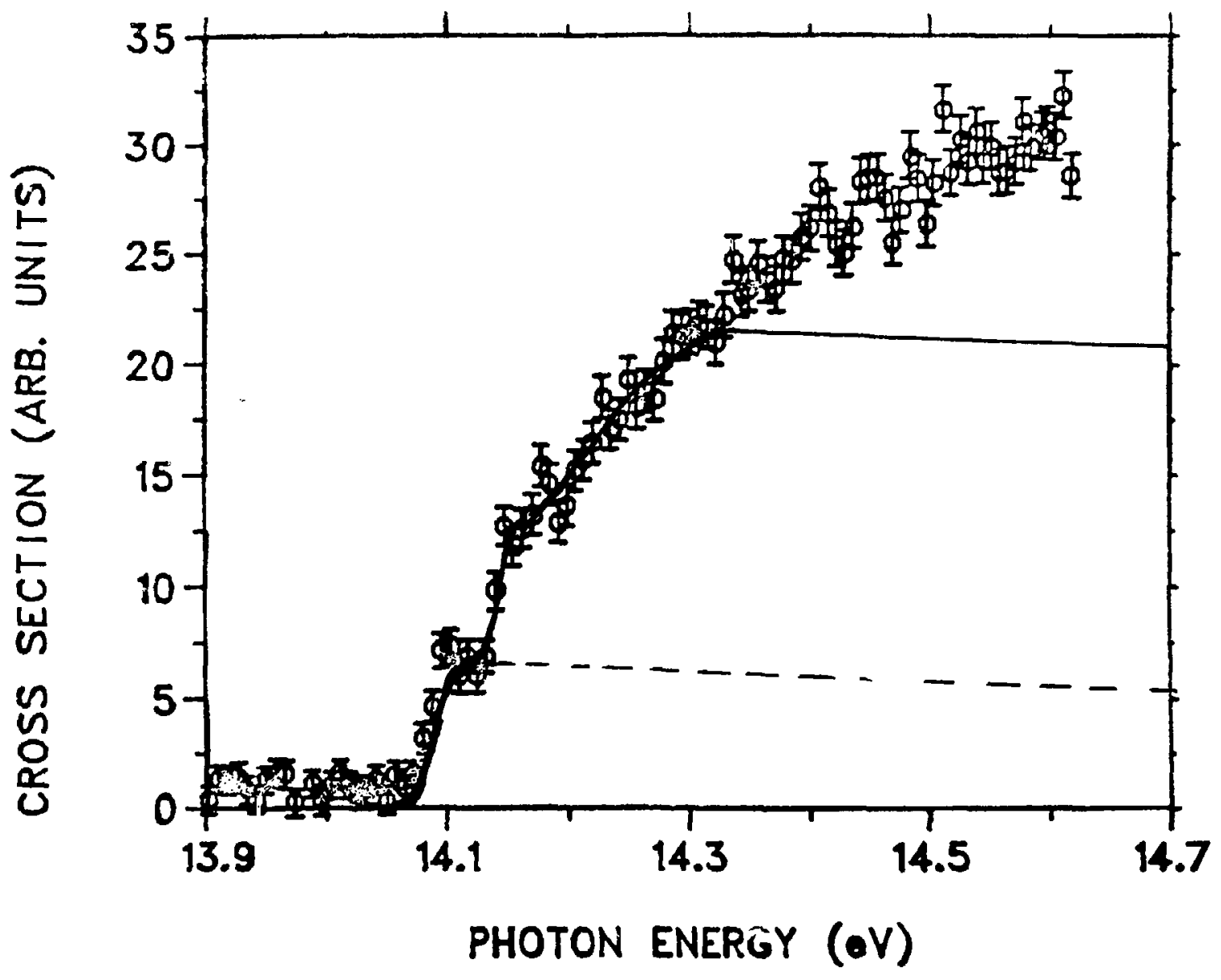

Figure 46. Fine Scan B $=780$ Gauss 
clearly visible with steps occurring as production of $\mathrm{H}^{\mathrm{O}}$ with $n=7,8$ and 9 becomes energetically allowed. For higher $n$, the steps are smeared together by the finite resolution of the apparatus.

The dashed curve is a hypothetical representation of the one-electron cross section for the single channel into $n=7\left(\gamma+\mathrm{H}^{-} \rightarrow \mathrm{H}^{\circ}(7)+\mathrm{e}^{-}\right)$. This curve is drawn by analogy to the channel into $\mathrm{n}=2$ which has been accurately calculated (See Figure 1 Chapter 1) by Broad and Reinhart (1978). The $n=7$ channel would rise sharply at $14.075 \mathrm{eV}$ and fall off very slowly with increasing energy. One can imagine the field stripped signal to be the superposition of all such curves for $n=7,8,9$, etc. This would generate the stair step structure we see. With increasing energy each step must be smaller in amplitude so that the sum will be finite at the series limit. The finite resolution of the apparatus blurs the stairstep into a smoothly rising curve. The curve continues to rise to the series limit as additional channels open up. Above the series limit no new states are available. We expect the field stripping cross section to fall off slowly with increasing energy above the series limit as each of its individual components does. The solid curve represents a hypothetical form for this total field stripped cross section. The continued increase in experimentaliy measured cross section above the series limit is assumed to be due 
to the two-electron photoejection process. This is confirmed by the 22 Gauss data where the field stripped contribution is reduced to a very small level while the two-electron signal is unaffected.

If we knew the exact form of the field stripped component we could substract this from the data to obtain the two-electron cross section over the extended energy range. We do not know the exact form but assume (by analogy to the channel into $n=2$ ) that it falls off slowly with increasing energy above $14.35 \mathrm{eV}$. Over a narrow energy range above the threshold we take the slowly varying function to be constant and subtract this constant from the experimental points to obtain the two-electron cross section near the threshold. When the separating field is reduced to 22 Gauss the background signal is much smaller than the desired signal so the approximation is surely justified in this case. There is also recent justification of the procedure on theoretical grounds (Temkin 1980).

Figure 47 shows similar data taken with a stripping field of 380 Gauss. The stair step structure is clearly visible. Steps at $n=8,9$ and 10 are resolved. The cross hatched area indicates the field ionization background assuming this to be constant above $14.35 \mathrm{eV}$. The twoelectron signal is now clearly resolved. One now has hope of determining the two-electron threshold law with further 


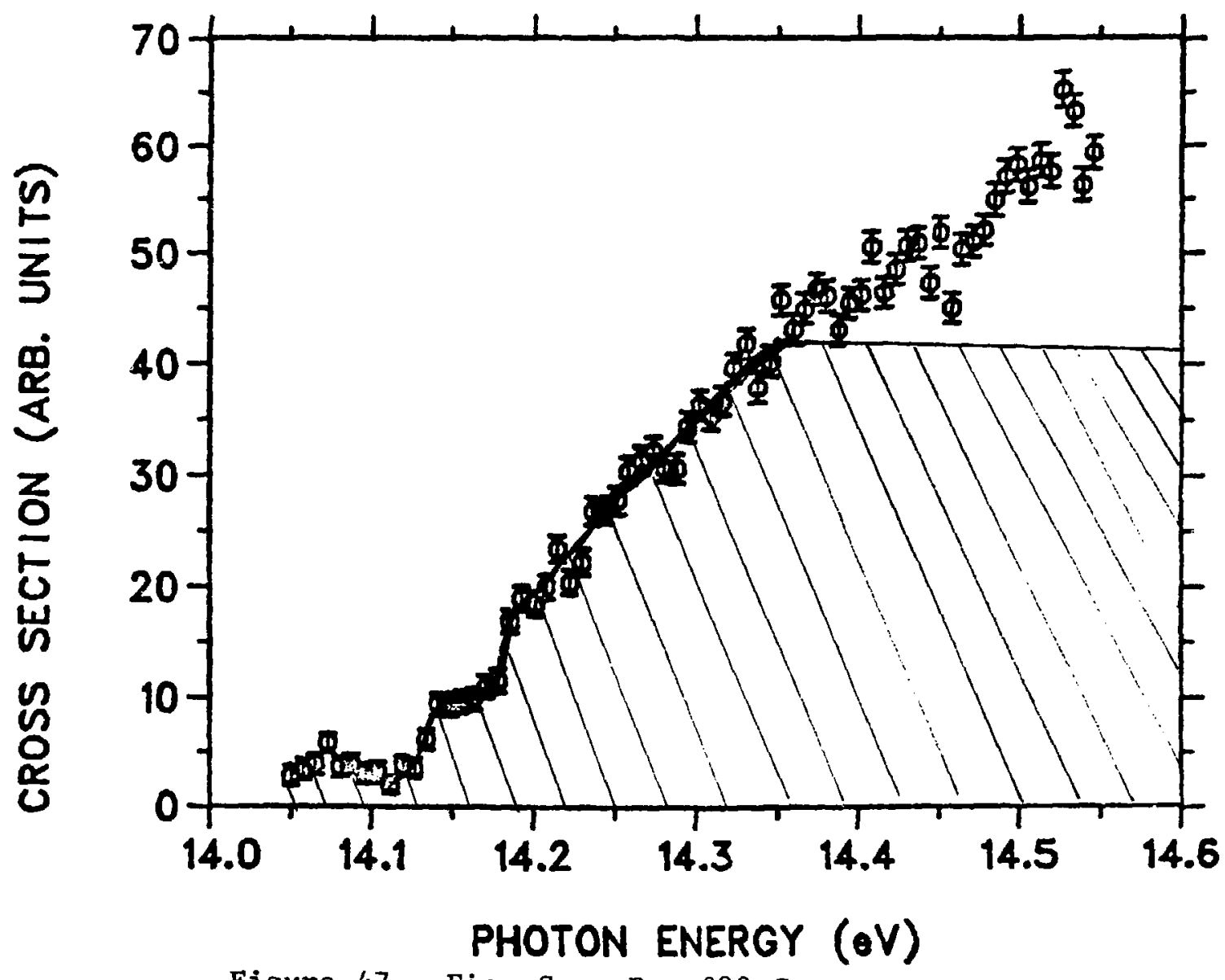

Figure 47. Fine Scan $B=380$ Gauss 
reduction in magnetic field. The following section discusses the data taken with the lowest field (22 Gauss) in detail.

E. Two-Electron Photoejection

The primary goal of experiment 449 was to observe two-electron photoejection from the $\mathrm{H}^{-}$ion. The cross section had been calculated (Broad and Reinhardt 1976) but the elusive process had never been observed experimentally previous to the present measurement. The field stripping problem was solved by reducing the deflection field to 22 Gauss allowing the two-electron photoejection cross section to be measured from threshold to $15.5 \mathrm{eV}$. This cross section was found to be in qualitative agreement with the calculation of Broad and Reinhardt, though probably somewhat greater in magnitude. In this section the general behavior is discussed and normalization relative to the one-electron cross section is obtained. The results are compared to the calculation of Broad and Reinhardt. The behavior very near the threshold is discussed in a later section.

Figure 48 displays the two-electron cross section calculated from counts in the $\mathrm{H}^{+}$detector. The minimum deflection field of 22 Gauss was employed to minimize the background due to field ionization. This scan (22C.Sum) is the result of adding all avallable data over the energy 


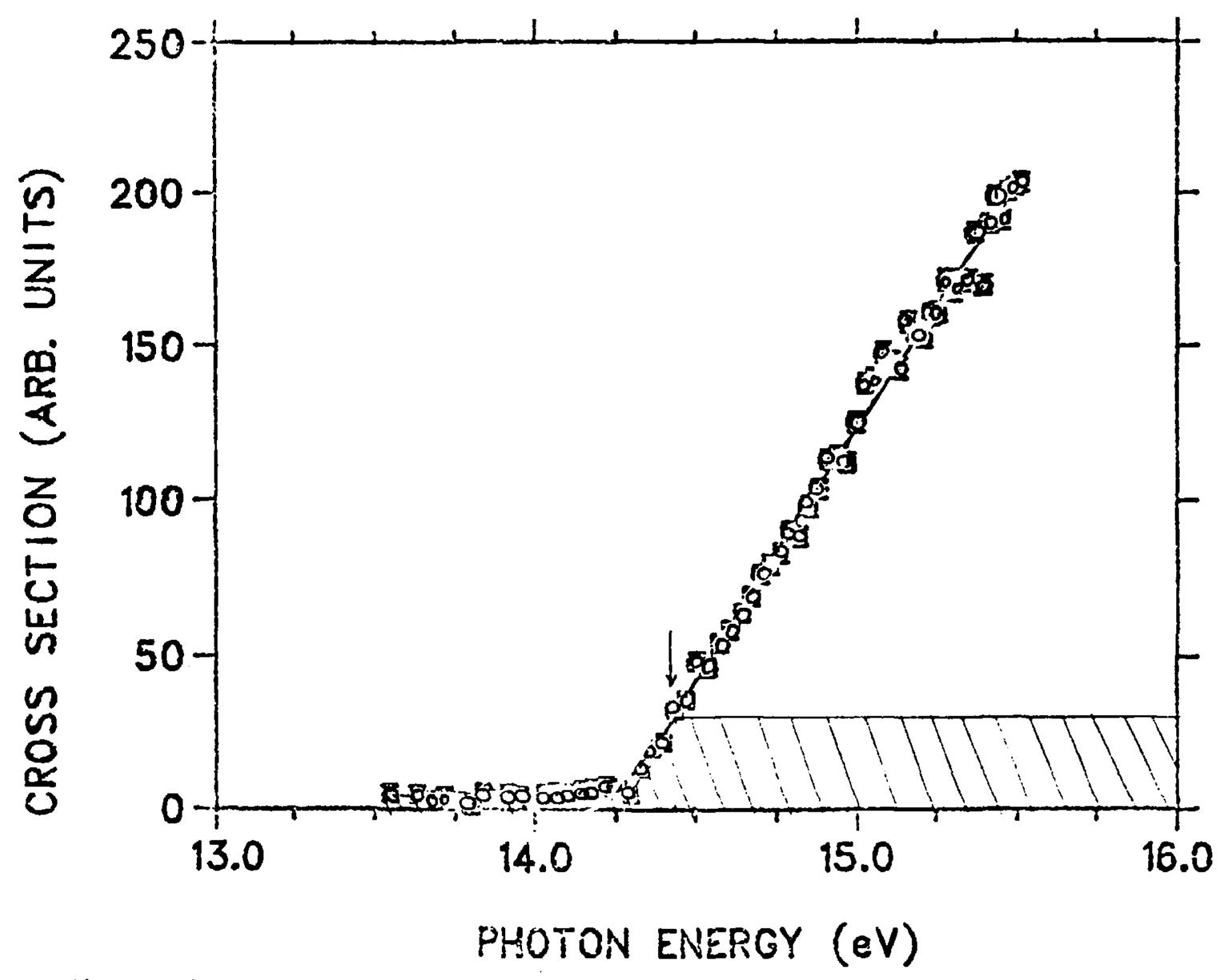

Figure 48. Two-electron Photoejection Cross Section from H+ Detector 
range of $13.5 \mathrm{eV}$ to $15.5 \mathrm{eV}$. The step size is 30.8 $\mathrm{meV} / \mathrm{step}$. Uncertainty in energy scale is estimated to be less than $15 \mathrm{meV}$ with instrumental resolution of $8 \mathrm{meV}$. This resolution is obtained by calculation relative to Feshbach scans (run numbers 2755, 2774 and 2786) as described in the section on calibration procedures. The vertical scale is arbitrary due to uncertainty in photon flux and beam overlap interval.

The scan which is due to both processes of equations 33 and 34, displays a sharp threshold just below $14.35 \mathrm{eV}$. This is the anamolous threshold due to field stripping. The two-electron cross section above $14.35 \mathrm{eV}$ rises almost linearly with increasing energy throughout the range of the scan. There is no indication that a peak is being approached. This is in agreement with Broad and Reinhart (1976) who predict a peak in cross section near $17 \mathrm{eV}$ but expect almost no decrease in slope below $16 \mathrm{eV}$. Their calculation (See Figure 4) shows the cross section rising steeply from threshold to $16 \mathrm{eV}$ where it folds over sharply.

The small constant background below the anamolous threshold appears to be due to ionization of excited $\mathrm{H}^{\mathrm{O}}$ 's by laser photons and collisions with residual gas molecules (enhanced gas stripping). The $\mathrm{H}^{\mathrm{O}} \mathrm{s}$ are of course produced by the one-electron process. Since the $\mathrm{H}^{\mathrm{O}}$ 's are produced in time with the laser flash, this background is 
not eliminated by the time coincidence requirement and accidental subtraction which corrects for normal gas stripping background.

The solid curve through the experimental points is obtained by fitting a power law to the data points. The form used is

$$
\sigma= \begin{cases}C\left[1+A\left(E-E_{O}\right)^{N}\right] & E \geq E_{O} \\ C & E<E_{O}\end{cases}
$$

This fit (22C09.FIT) gives,

$$
\begin{aligned}
\mathrm{N} & =0.940 \pm 0.018 \\
E_{O} & =14.293 \mathrm{eV} \pm 0.009 \mathrm{eV} \\
\mathrm{A} & =37.0 \pm 5.4 \\
\mathrm{C} & =4.47 \pm 0.57 \\
\mathrm{x}^{2} & =130.4 \text { for } 6: \text { degrees of freedom }
\end{aligned}
$$

The threshold $\left(E_{O}\right)$ at $14.293 \mathrm{eV}$ corresponds with the field stripping threshold for $n=15$. The uncertainty in energy scale and fit expands the range to include $n_{c}$ from 13 to 17 . This is consistant with the calculated value of $\mathbf{n}_{\mathrm{c}}=14$ for the 22 Gauss field. The background constant $\mathrm{C}$ is required to correct for background below the threshold, which should be considered as an instrumental effect rather than part of the two-electron cross section. Evaluating equation 35 at $14.353 \mathrm{eV}$ gives the total background from all sources since the two-electron cross 
section is zero at this energy. This is of course subject to the uncertainty in energy scale. We have,

Background $=4.47\left[1+37.0(14.353-14.293)^{.940}\right]=16.2$

This constant must be subtracted from the data above threshold to obtain the two-electron corponent. The cross hatched area indicates the background assumed to be constant above $14.353 \mathrm{eV}$. This procedure appears reasonable as discussed in the previous section since the small background falls off very slowly with increasing energy. The background is clearly much smaller than the twoelectron signal.

The convenient parameterization is not exactly correct. The exponent above $14.35 \mathrm{eV}$ may be different from that below since they are the result of entirely different processes. Fitting the same form to only the points above threshold gives the exponent for the twoelectron process alone. The fit above $14.35 \mathrm{eV}$ gives,

$$
\begin{aligned}
& N=0.946 \pm 0.025 \\
& \text { Eo }=14.35 \mathrm{eV} \text { Fixed } \\
& A=11.0 \pm 1.7 \\
& C=14.9 \pm 1.8 \\
& x^{2}=111.9 \text { for } 39 \text { degrees of freedom }
\end{aligned}
$$

No significant change in $N$ is seen. This is to be expected since the field stripped background region 
consisted of only three data points and could not affect the shape of the curve significantly. The background constant obtained by this fit agrees within errors bars to that calculated using the whole scan. The $x^{2}$ per degree of freedom is increased since the small constant background below threshold, where the fit was very good, has been excluded.

The value found for the exponent $(0.95 \pm 0.02)$ is smaller than the expected Wannier value of 1.127 by 7 standard errors. This probably indicates that the range of $1.16 \mathrm{eV}$ above threshold which was examined is larger than the range of validity for the threshold law. The decreasing slope at high energy might also be caused by laser beam wander.

To investigate this hypothesis and also obtain a normalization of the two-electron cross section relative to the one-electron cross section, we study the ratio of $\mathrm{H}^{+}$ signal to $\mathrm{H}^{\mathrm{O}}$ signal as a function of energy. The ratio of $\mathrm{H}^{+}$scintillator counts to $\mathrm{H}^{\mathrm{O}}$ counts should be completely independent from the effects of laser beam wander since both count rates would be equally depressed if the overlap of the beams was reduced.

Figure 49 plots the ratio (HPLHZE cross section) for the data of the previous scan. The energy uncertainty and resolution are unchanged since this plot is of the same 


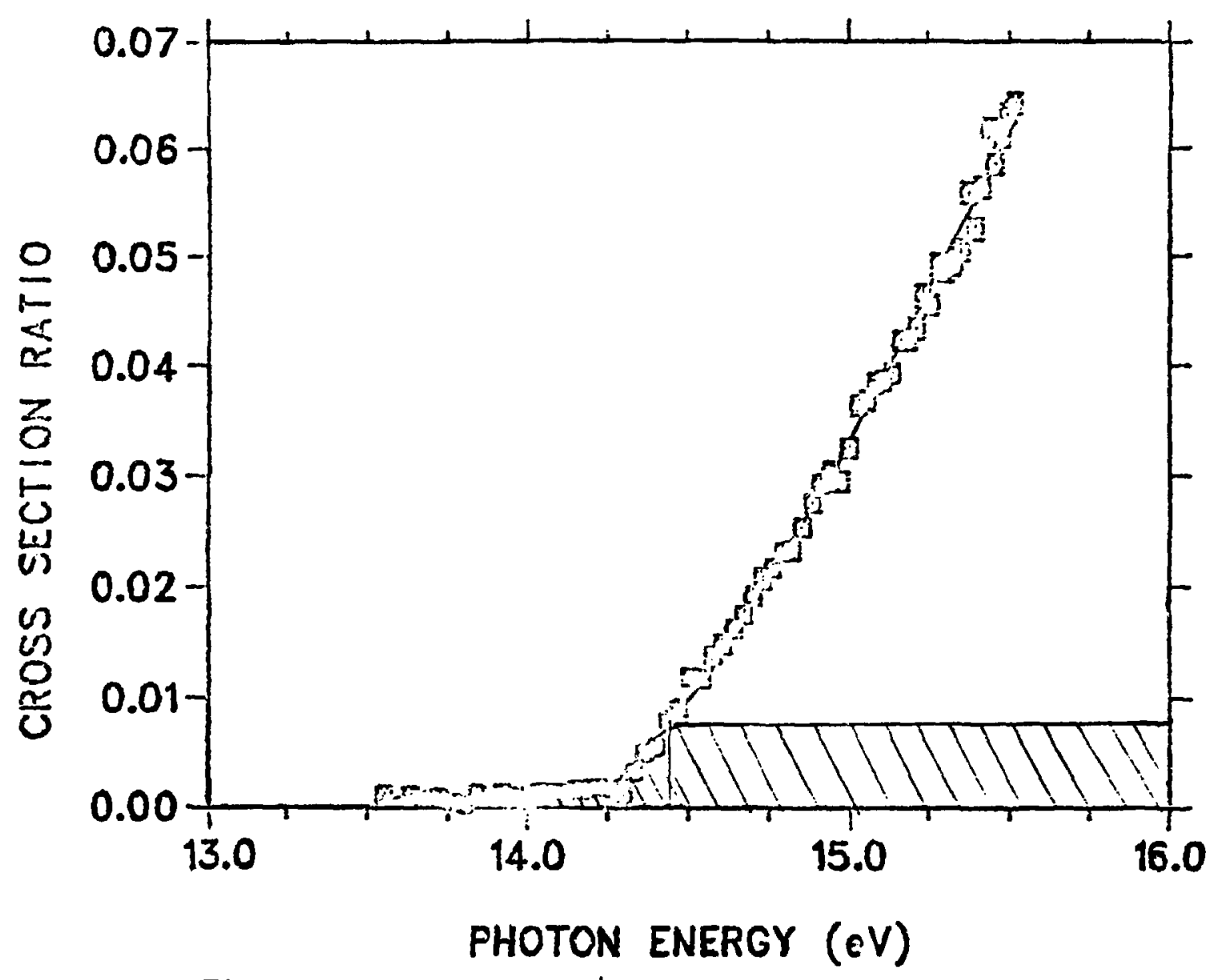

Figure 49. Ratio of $\mathrm{H}^{+}$Signal to $\mathrm{H}^{\circ}$ Signal 
scan. The uncertainty in vertical scale is approximately a factor of 2 based on variations in repeated measurements. This uncertainty in the ratio results from detector nonlinearity and variations in collection effiencies of the scintillators as the incident beam drifts in entrance angle.

Steering the photodetached beam onto the tiny scintillator some twenty meters downstream of the interaction chamber is exceedingly difficult. After this is accomplished, slight variations in entrance angle of the ion beam to our apparatus cause motion of the photodetached beam on the $3 / 8^{\prime \prime}$ scintillator. These motions create variations in collection efficiency. Much of the variation also appears to be due to nonlinearity in the $\mathrm{H}^{\mathrm{O}}$ detector.

As in the previous figure, the cross hatched region represents the background assumed constant above $14.35 \mathrm{eV}$. The solid curve is the fit of a power law using equation 35. The results of this fit are

$$
\begin{aligned}
\mathrm{N} & =1.30 \pm 0.03 \\
\mathrm{E}_{O} & =14.202 \mathrm{eV} \pm 0.023 \mathrm{eV} \\
\mathrm{A} & =45.34 \pm 0.45 \\
\mathrm{C} & =9.557 \times 10^{-4} \pm 9.7 \times 10^{-6} \\
\mathrm{x}^{2} & =125.7 \text { for } 61 \text { degrees of freedom. }
\end{aligned}
$$


Fitting only points above $14.35 \mathrm{eV}$ gives:

$$
\begin{aligned}
N & =1.250 \pm 0.025 \\
E_{O} & =14.35 \mathrm{eV} \text { Fixed } \\
A & =8.20 \pm 0.63 \\
C & =5.74 \times 10^{-3} \pm 3.7 \times 10^{-4} \\
x^{2} & =101.0 \text { for } 39 \text { degrees of freedom. }
\end{aligned}
$$

The fit to $\left(\mathrm{H}^{+} / \mathrm{H}^{\circ}\right)$ indicates the anamolous threshold to be lower by $91 \mathrm{meV}$ than that given by the fit to the $\mathrm{H}^{+}$signal. This is almost three standard deviations disagreement. Inspection of the curves indicates the lower value of the fit to the $\mathrm{H}^{+}$channel is probably more correct. Fitting to the ratio misses points near the threshold and seems to give a value which is too low in energy for the sharp threshold.

The exponents also differ greatly. With the ratio giving a val.e of 1.25 compared to 0.95 for the $\mathrm{H}^{+}$ channel. This disagreement is 7 standard errors. The disagreement is visible by examination of the two curves. The major disagreement occurs for the highest energies where the slope of the $\mathrm{H}^{+}$curve is almost linear or possibly starting to fold over slightly while the ratio $\left(\mathrm{H}^{+} / \mathrm{H}^{\circ}\right)$ shows increasing slope for the highest energies. The cause of the problem appears to be saturation of the $\mathrm{H}^{\mathrm{O}}$ detector for the very highest energies where the angle is small. 
Figurs 35 shows the $\mathrm{H}^{\circ}$ signal which was used in the ratio. N tice the droop in cross section above $15.2 \mathrm{eV}$. As previously discussed in section B, this droop appears to be caused by saturation of the $\mathrm{H}^{\circ}$ detector at small angles (high energy) where the geometric factor causes a large increase in counting rate. This conclusion is verified by a scan (run 2791) which was taken over the same energy region with the beam current reduced by an order of magnitude. The ratio at the highest energy $(15.5 \mathrm{eV})$ is increased by a factor of two with respect to that at the lower beam current. The ratio should have been immune to changes in beam current if both channels had been linear. This run gives a clear indication of nonlinearity or saturation of the $\mathrm{H}^{\circ}$ channel. This saturation accounts for much of the uncertainty in vertical scale on the ratio. With saturation in the $\mathrm{H}^{\mathrm{O}}$ channel, the ratio will vary from run to run and will always read higher than the true value.

By taking the ratio of the two-electron cross section to one-electron cross section from Broad and Reinhardt (see figure 5), we obtain the ratio 0.02 at $15.5 \mathrm{eV}$. This compares with our measured value of 0.062 . This disagreement could be explained by the saturation problem and uncertainty due to motion of the photodetached beam on the scintillator. The values of Broad and Reinhardt are 
also quite uncertain. They predict a peak of approximately $5 \times 10^{-3}$ square Bohrs somewhere near $17 \mathrm{eV}$ but are unsure of the exact energy of the peak or the magnitude of the cross section (Reinhardt 1980). Their calculations: are not well converged below $22 \mathrm{eV}$.

Multiplication of the experimental ratio by the calculated one-electron cross section gives us normalization relative to the better understond theory of the one-electron cross section. This is done by multiplying each point of figure 49 by the theoretical one-alectron. cross section for the same energy obtained from a cubic spline fit to the continuum points of Broad and Reinhardt. Figure 50 shows the result of this procedure which gives the two-electron cross section expressed in units of square Bohrs. These points are also presumably high due to saturation of the $\mathrm{H}^{\circ}$ detector. These are in agreenent with Broad and Reinhardt within the wide uncertainty in both theory and experiment.

The thsory can surely be tested more stringently if this scan is repeated with more attention to prevent saturation of the $\mathrm{H}^{\mathrm{O}}$ detector. Even a single point taken at the highest energy with linearity demonstrated by independence of the ratio $\left(\mathrm{H}^{+} / \mathrm{H}^{\circ}\right)$ to variation of beam current and photon flux would allow accurate normalization. Due to the saturation problem the shape of the $\mathrm{H}^{+}$scan (figure 48) is probably the most accurate of the 


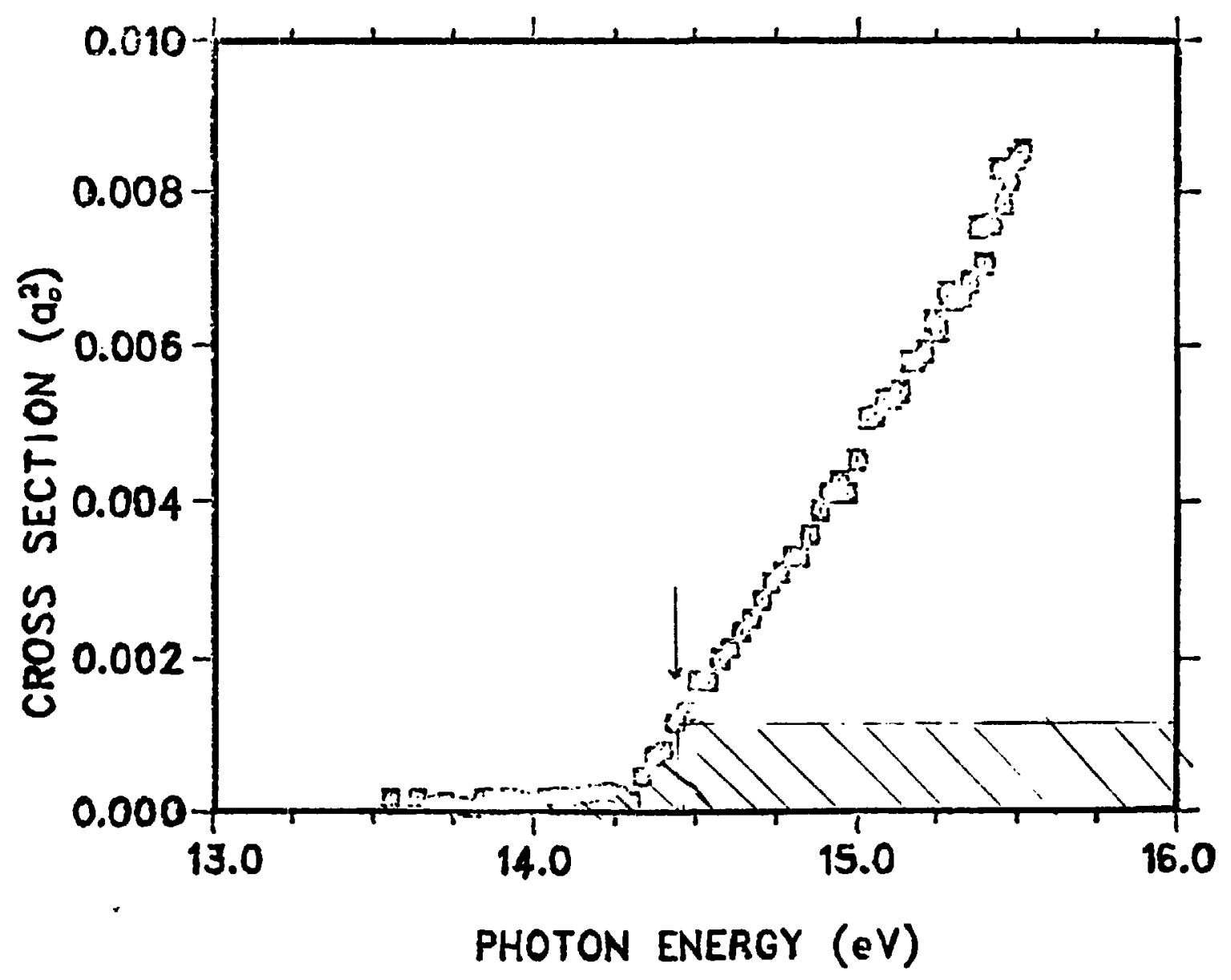

Figure 50. Two-Electron Cross section Normalized to the One-Electron Cross Section Calculation of Broad and Reinhardt 
three figures. The values in figure 50 are probably somewhat high but even if they are high by a factor of two, the measured cross section is still considerably higher than the theory. This may be a matter of great significance to the astrophysicist. Due to its importance we should repeat this measurement with care to prevent saturation and using the 5 th harmonic crystal to get higher photon energy and find the peak of the curve.

F. Two-Electron Threshold

In this section the two-electron threshold is examined in detail. Only data taken with the minimum deflection field of 22 Gauss are considered. These data will be the least affected by the field-stripped background and thus should give the best representation of the true twoelectron threshold. All available fine scans over the threshold were combined into one master scan (22F.SUM). The total running time to acquire this scan was approximateily 10 hours.

The resulting scan covered the energy range of 14.050 $\mathrm{eV}$ to $14.545 \mathrm{eV}$ with a step size of $7.17 \mathrm{meV}$. The calibration of energy and resolution is identical with that for the course scan of the previous section with an uncertainty in energy scale of $15 \mathrm{meV}$ based on the largest error observed in the location of the stripping threshold 
in previous high field scans. The instrumental resolution is calculated (as described in the section III-L) to be 8 meV.

Since the two electron cross section approaches zero at the threshold the counting rate is very small near the threshold. Even with ten hours of counting time the statistical sample is inadequate to provide the desired precision in the measurement of the threshold law. Much more time was scheduled, but it was only in the final hours of the experiment that the field stripping was reduced to an acceptable value allowing the measurement to be completed.

A power law fit to the data agrees with the Wannier law (1953). The result is also, within the large error bars, consistent with a linear law. The data was also compared with the modulated linear law predicted by Temkin (1974). It appears that Temkin's form fits no better than the Wannier law. Limits are placed on the magnitude of the oscillations predictêd by Temikin.

Due to the importance of the two-electron threshold data, considerable analysis was performed on this scan. Despite these efforts $\Delta N / N$ is determined to only $15 \%$. This is due to the limited statistical base available. The methods are described in detail as the prototype for future analysis. The validity of the method has been demonstrated. Using data with better statistics it should 
be possible to obtain $\Delta N / N$ to much higher precision. It will however be essential to reduce systematic errors in order to take advantage of improved statistics.

Figure 51 shows the fine scan over the two-electron threshold with cross section calculated from the $\mathrm{H}^{+}$ detector. The vertical scale is arbitrary but is identical to the scale used for the coarse scan discussed in the previous section. Error bars are calculated from counting statistics only.

The anomalous threshold due to field stripping is clearly visible below $14.35 \mathrm{eV}$. The constant background below this threshold is assumed to be due to ionization of $\mathrm{H}^{\circ}$ 's by laser photons and enhanced gas stripping. Cross hatching indicates the total background assuming a constant background due to field stripping above 14.353 eV. Background is more apparent in this scan than it was in the coarse scan due to the very small two-electron rate near the threshold. For the same reasnn the fractional error bars are larger.

The solid curve is the best fit of a power law of the form of equation 35 giving

$$
\begin{aligned}
\mathrm{N} & =0.93 \pm 0.10 \\
\mathrm{E}_{\mathrm{O}} & =14.292 \pm 0.001 \mathrm{eV} \\
\mathrm{A} & =29.8 \pm 4.8
\end{aligned}
$$




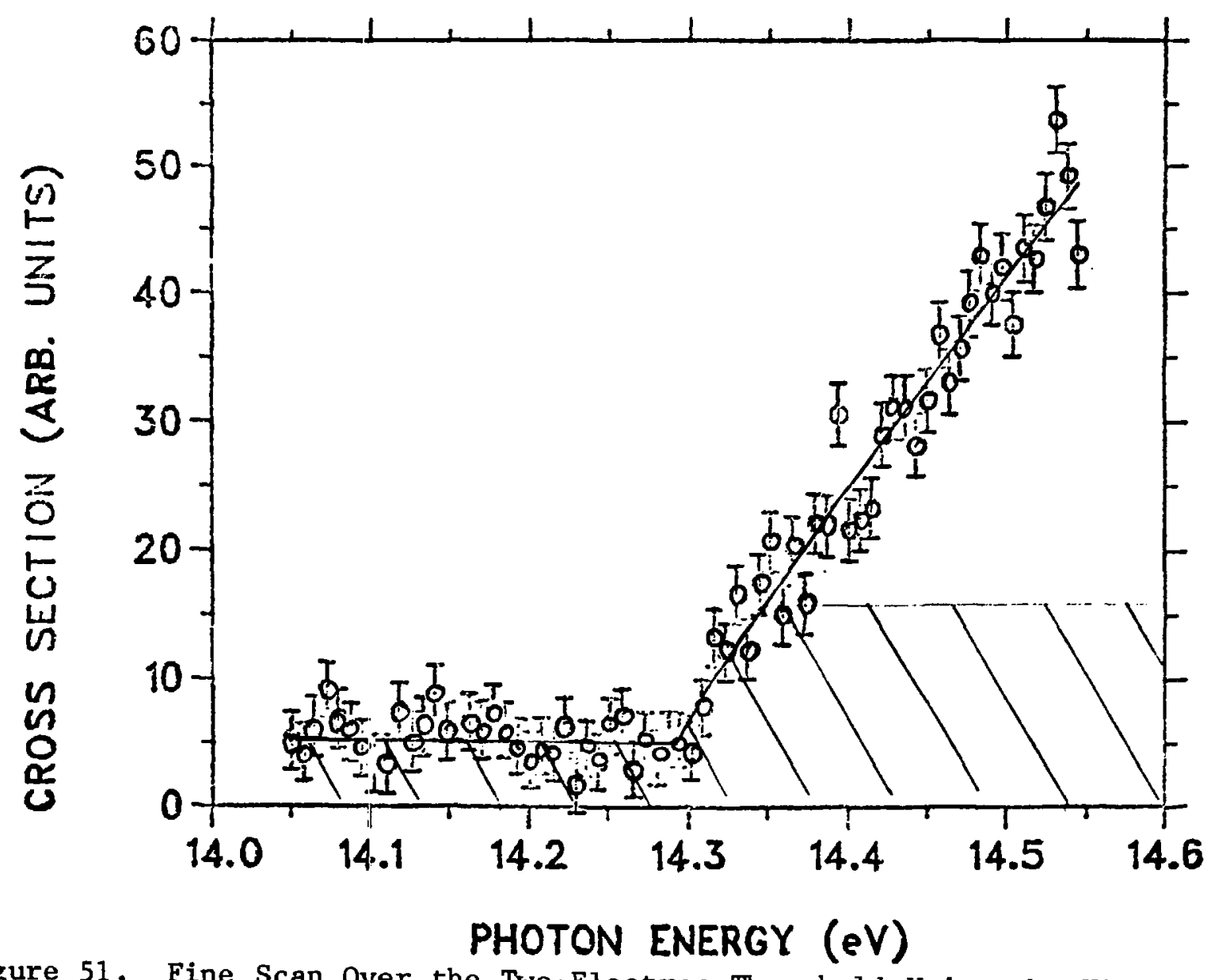

Figure 51. Fine Scan Over the Two-Electron Threshold Using the $\mathrm{H}^{*}$ Detector 


$$
\begin{aligned}
& C=5.23 \pm 0.38 \\
& x^{2}=83.6 \text { for } 66 \text { degrees of freedom. } \\
& \text { Confidence Level }=0.06
\end{aligned}
$$

Figure 52 shows the ratio of $\mathrm{H}^{+}$signal to the $\mathrm{H}^{\circ}$ signal over the same energy range. Here the vertical scale corresponds approximately to the two-electron cross section relative to the one electron cross section. The vertical scale is consistent with the coarse scan of the previous section.

The solid curve is the best fit of a power law to the data. This gives

$$
\begin{aligned}
& N=0.914 \pm 0.099 \\
& E_{O}=14.293 \pm 0.010 \\
& A=31.0 \pm 4.9 \\
& C=1.266 \times 10^{-3} \pm 9.3 \times 10^{-5} \\
& x^{2}=76.4 \text { for } 66 \text { degrees of freedom. } \\
& \text { Confidence Leve1 }=0.2
\end{aligned}
$$

The curves of figure 51 and 52 appear identical in shape. The parameters obtained by fitting are almost identical except for the vertical scale factor. This indicates that the problem with laser beam wander which was seen in the coarse scan is not significant for the fine scan. This problem may be ignored over the small angular range covered in the fine scan since the cross section changes much more rapidly with angle than the overlap. 


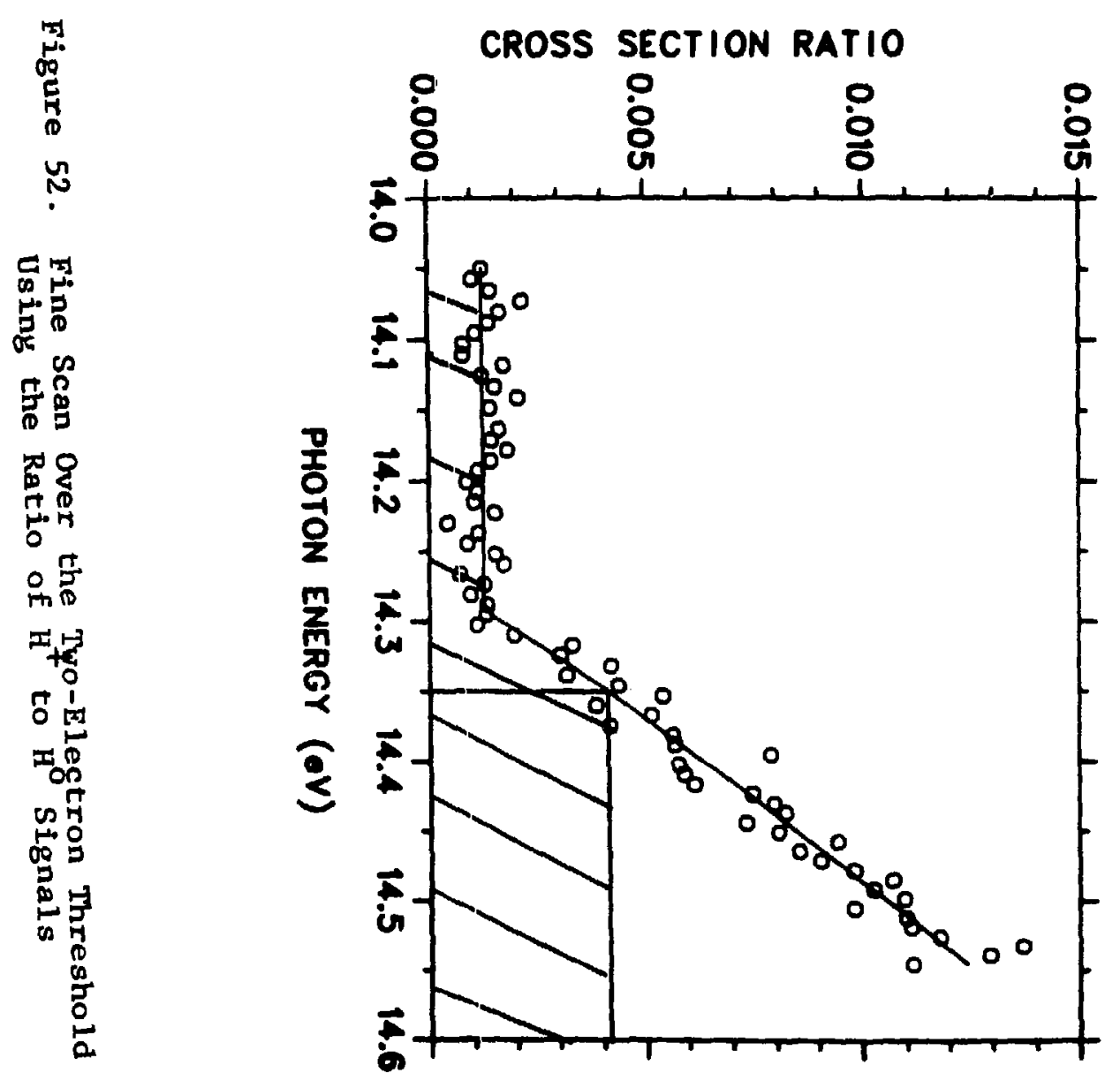

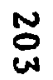


The parameter values found from fitting the fine scan are seen to agree quite closely with those obtained from the coarse scan. The anomalous threshold at $14.293 \mathrm{eV}$ corresponds with field stripping threshold for $n=15$. The uncertainty in energy scale and fit expands the range to include $\mathrm{n}_{\mathrm{c}}$ from 13 to 17 . This is consistent with the calculated value of $\mathrm{n}_{c}=14$ for the 22 Gauss field. The magnitude of the total background assumed to be constant above $14.353 \mathrm{eV}$ may be obtained br evaluation of equation 35 at threshold. This gives a background of 16.8 for the $\mathrm{H}^{+}$signal and $4.27 \times 10^{-3}$ for the $\left(\mathrm{H}^{+} / \mathrm{H}^{\circ}\right)$ channel. These constants must be subtracted from the respective experimental points above $14.35 \mathrm{eV}$ to obtain the true twoelectron cross section. This is of course subject to the uncertainty in energy scale.

The background data in the region below the anomalous threshold at $14.29 \mathrm{eV}$ was fit by a constant, in order to investigate the legitimacy of using statistical error bars. The result is a background constant of $5.20 \pm .37$ for the $\mathrm{H}^{+}$channel and $(1.26 \pm .09) \times 10^{-3}$ for the $\left(\mathrm{H}^{+} / \mathrm{H}^{\circ}\right)$ channel. In both cases the $x^{2}$ was slightly less than one per degree of freedom indicating that the error bars are not underestimated for this scan.

The simple parameterization which we have obtained gives close agreement to that obtained from the coarse 
scan and also fits our data and background well. It does not, however, correctly express the true two-electron threshold law since it treats the field stripped background below $14.35 \mathrm{eV}$ exactly the same as the twoelectron cross section above. This would be acceptable if the application of the stripping field simply pushed the threshold energy lower without changing the shape of the curve. Actually we expect a different exponent below the two-electron threshold since a different process is acting here.

To obtain separation of the true two-electron threshold law from the background we fit only the points above $14.35 \mathrm{eV}$ as was done with the coarse scan in the previous section. The gradual drop in background with increasing energy will be closely approximated by the background constant over the narrow energy range covered by the fine scan.

Figure 53 shows the cross section for points above the two-electron chreshold calculated from the $\mathrm{H}^{+}$detector signal. The solid curve is the best fitting curve of the form :

$$
\sigma=B+A\left(E-E_{O}\right)^{N}
$$

where $\sigma$ is the measured cross section, B is the total background assumed to be constant above $14.35 \mathrm{eV}, \mathrm{A}$ is the scale factor $\mathrm{N}$ is the exponent in the power law and $\mathrm{E}$ is 


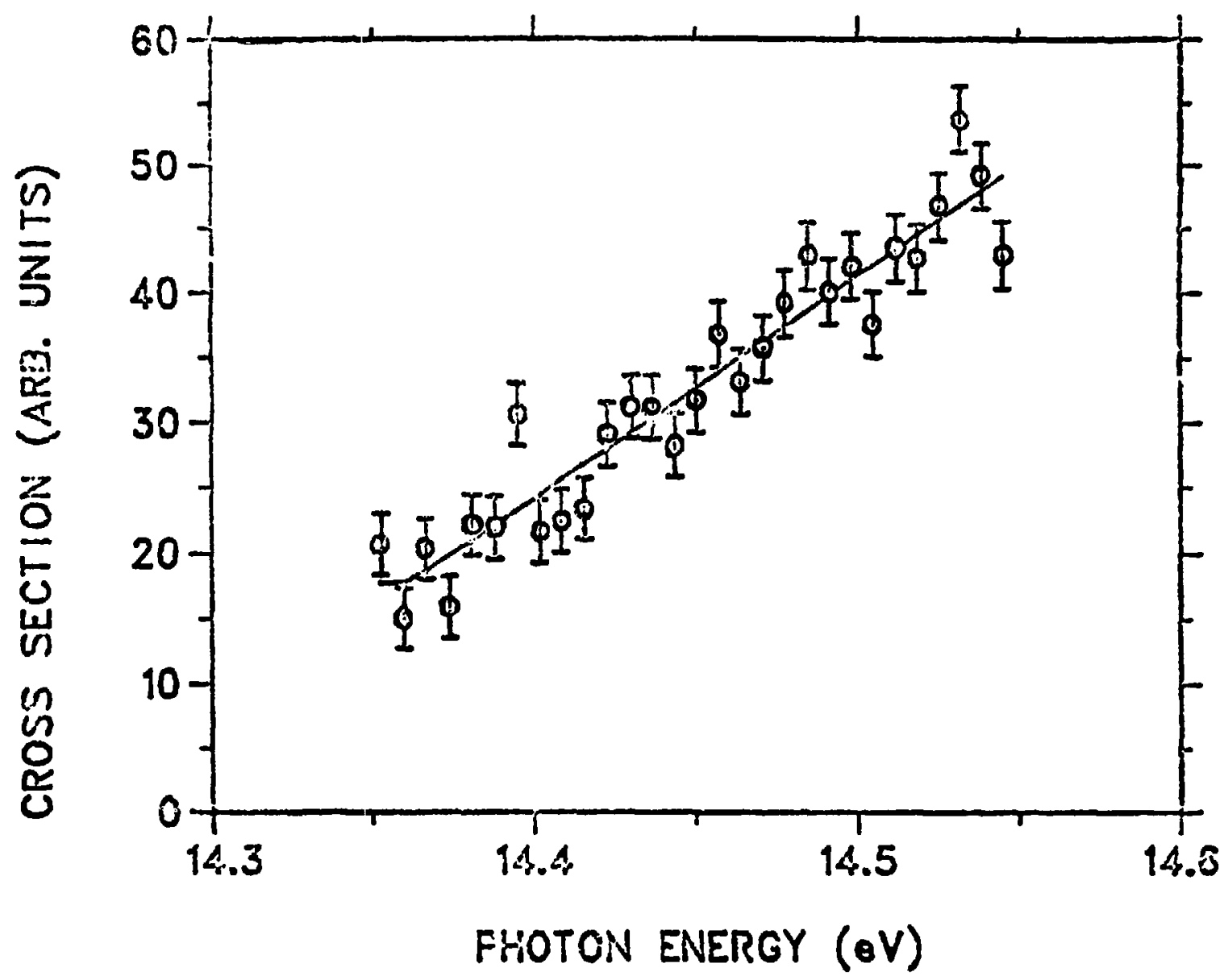

Figure 53. Two-Electron Cross Section Calculated from H+ Detector Using Points Above $14.35 \mathrm{eV}$ only 
the photon energy. $E_{0}$ is the threshold energy which is allowed to be a free parameter due to the large uncertainty in energy scale. The results of fitting are

$$
\begin{aligned}
& N=1.03 \pm 0.14 \\
& E_{O}=14.361 \mathrm{eV} \pm 0.003 \mathrm{eV} \\
& A=178 \pm 42 \\
& B=17.8 \pm 1.2 \\
& x^{2}=46.1 \text { for } 25 \text { degrees of freedom. } \\
& \text { Confidence Level }=0.008
\end{aligned}
$$

Fitting the same form to the ratio of $\mathrm{H}^{+}$to $\mathrm{H}^{\mathrm{O}}$ signals above threshold gives

$$
\begin{aligned}
& N=1.03 \pm 0.16 \\
& E_{O}=14.360 \mathrm{eV} \pm .004 \mathrm{eV} \\
& A=0.045 \pm 0.011 \\
& B=4.59 \times 10^{-3} \pm 4.2 \times 10^{-4} \\
& X^{2}=40.3 \text { for } 25 \text { degrees of freedom. } \\
& \text { Confidence Leve1 }=0.02
\end{aligned}
$$

This curve is seen in figure 5i. The two curves agree alnost exactly except for the vertical scale. This agreement gives us confidence that both $\mathrm{H}^{+}$and $\mathrm{H}^{\circ}$ signals are free of saturation and that there is no problem with laser bean wander over the narrow angular range of the scan. Eo differs by $7 \mathrm{meV}$ from the theoretical value of $14.353 \mathrm{eV}$. This difference is well within the uncertainty in energy scale and is actually less than the step size. 


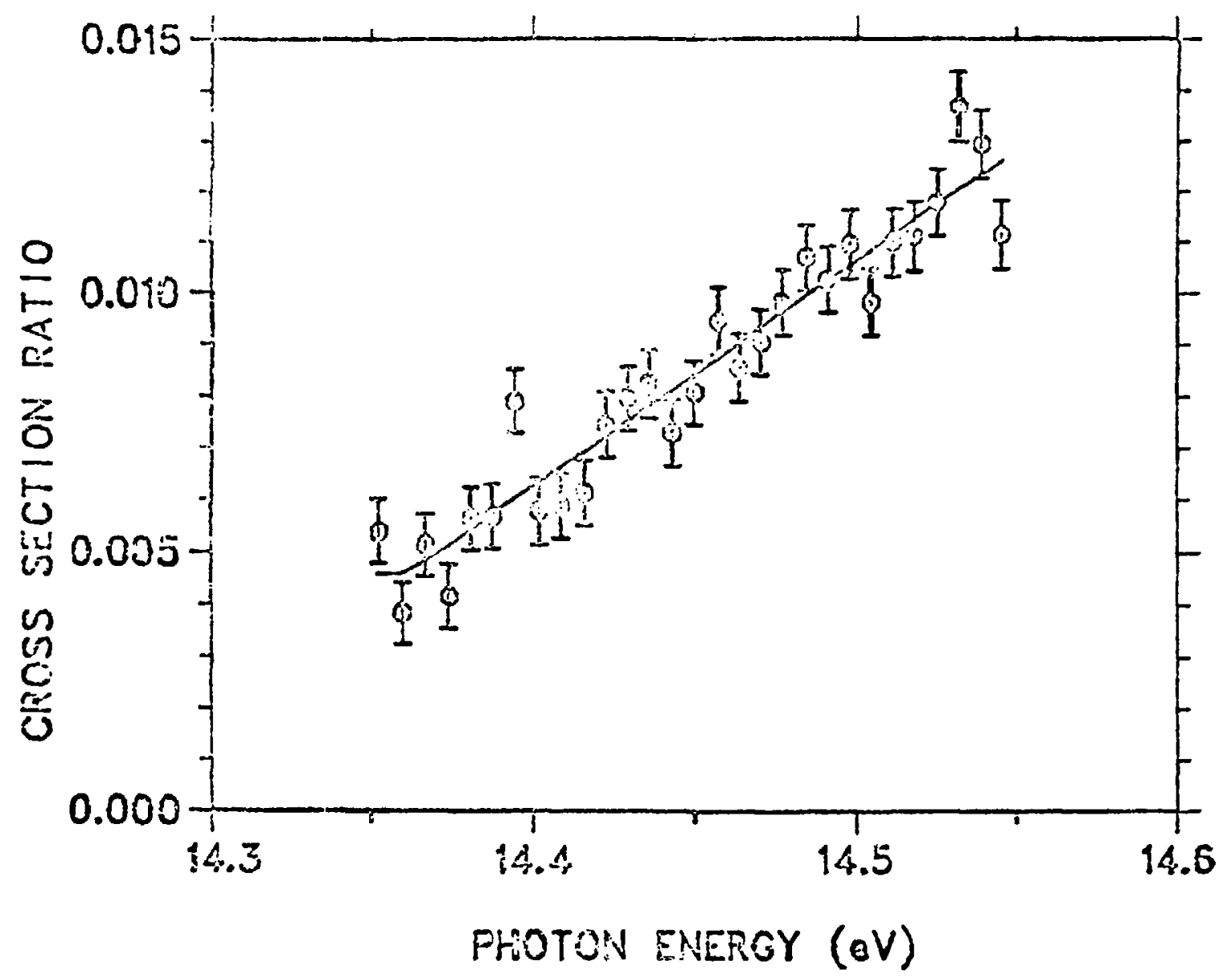

Figure 54. Ratio of $\mathrm{H}^{+}$to $\mathrm{H}^{\mathrm{O}}$ Signal Above $14.35 \mathrm{eV}$ 
It is interesting to note that $\mathrm{E}_{\mathrm{O}}$ is found near the two-electron threshold rather than near the anomalous threshold. If the effect of the field stripping were simply to push the threshold energy down without changing the shape of the curve we might expect the fitting routine to extrapolate to $E_{0}$ near $14.29 \mathrm{eV}$. This is not the case.

The value of $1.03 \pm .15$ obtained for $N$ is consistent with the Wannier law as well as a linear threshold law. The error bars are large due to the poor statistics of the data and the difficulty of trying to find $E_{O}$ with no clear intercept. This difficulty is reflected in the high correlation coefficients. Both fits above threshold give a global correlation coefficient (see Section A) of 0.994 on N. The correlation is tightest (correlation coefficient 0.97) to $A$ but is also quite high (correlation coefficient 0.7 ) to $\mathrm{E}_{\mathrm{O}}$. We cannot know $\mathrm{A}$ a priori. A must be a free parameter. We could, however, fix $E_{O}$ at $14.353 \mathrm{eV}$ if we had a sufficiently accurate energy calibration.

Fitting the data of figure $52 \mathrm{CH}^{+}$cross section above threshold) with $E_{O}$ fixed at $14.35 \mathrm{eV}$ gives $\mathrm{N}=1.10 \pm .15$ with $\mathrm{x}^{2}=47.4$.

The same procedure with the data of figure 27 $\left(\mathrm{H}^{+} / \mathrm{H}^{\circ}\right.$ above threshold) gives $\mathrm{N}=1.11 \pm .14$ with $\mathrm{x}^{2}=$ 41.5. The global correlation coefficient on $\mathrm{N}$ is reduced from .994 to .95 by fixing $E_{O}$. In a similar manner $E_{O}^{\prime}$ is fixed at the upper and lower energy limits corresponding 
with an energy uncertainty of $15 \mathrm{meV}$. These energy limits translate into limits on $N$. To these appropriate fitting error on $\mathrm{N}$ is added. This gives $\mathrm{N}=1.10_{-.16}^{+.21}$.

This result is closer to the expected Wannier value than that obtained with $E_{0}$ free but the error bars on $N$ are actually larger when the uncertainty in energy calibration is considered.

An attempt was made to determine the range of validity of the law above $14.35 \mathrm{eV}$ as was done with the oneelectron threshold law. This was found to be impossible due to poor statistics and the limited number of data points above the two-electron threshcid. The curve fitting routine would not converge properly for any less than the full set of 29 points above the two-electron threshold. This set corresponds to an energy range of $192 \mathrm{meV}$.

The field stripped background below the two-electron threshold is due to a different process than the twoelectron photoejection. We expect a different exponent in the threshold law in this region. In order to demonstrate this behavior a parameterization was constructed which allowed separate exponents above and below the twoelectron threshold. The form employed was

$$
\sigma=\left\{\begin{array}{lc}
C\left[1+A_{1}\left(E_{o}-E_{n}\right)^{N_{1}}+A_{2}\left(E-E_{0}\right)^{N_{2}}\right] E>E_{o} \\
C\left[1+A_{1}\left(E-E_{n}\right)^{N 1}\right] \quad E_{o} \geq E>E_{n} \\
C & E_{n} \geq E
\end{array}\right.
$$


where $\sigma$ is the measured cross section, $E$ is the photon energy, $E_{0}$ the two-electron threshold, $E_{n}$ the field stripping threshold corresponding with $n_{c} \cdot N_{1}$ and $N_{2}$ and the exponents in the threshold power law correspond to stripping background and two-electron photoejection respectively. The result of fitting to the data from the ratio $\left(\mathrm{H}^{+} / \mathrm{H}^{\circ}\right)$ channel is shown in figure 55 . The parameters found are

$$
\begin{aligned}
& \mathrm{N}_{1}=0.53 \pm 0.21 \\
& \mathrm{~N}_{2}=1.09 \pm 0.11 \\
& \mathrm{E}_{\mathrm{n}}=14.3029 \mathrm{eV} \pm 0.0024 \mathrm{eV} \\
& \mathrm{E}_{\mathrm{O}}=14.35 \mathrm{eV} \text { Fixed } \\
& \mathrm{A}_{1}=12.5 \pm 8.9 \\
& \mathrm{~A}_{2}=39.3 \pm 7.6 \\
& \mathrm{C}=1.262 \times 10^{-3} \pm 8.8 \times 10^{-5} \\
& x^{2}=71.5 \text { for } 63 \text { degrees of freedom. } \\
& \text { Confidence Level }=0.2
\end{aligned}
$$

This form fits the data well. $x^{2}$ per degree of freedom is lower here than for any other form tried. This fit is consistent with the previous fits in the determination of the power law above the threshold for two-electron photoejection. Below this threshold we have a new threshold law associated with the one-electron photodetachment into Rydberg states. The error bars on 


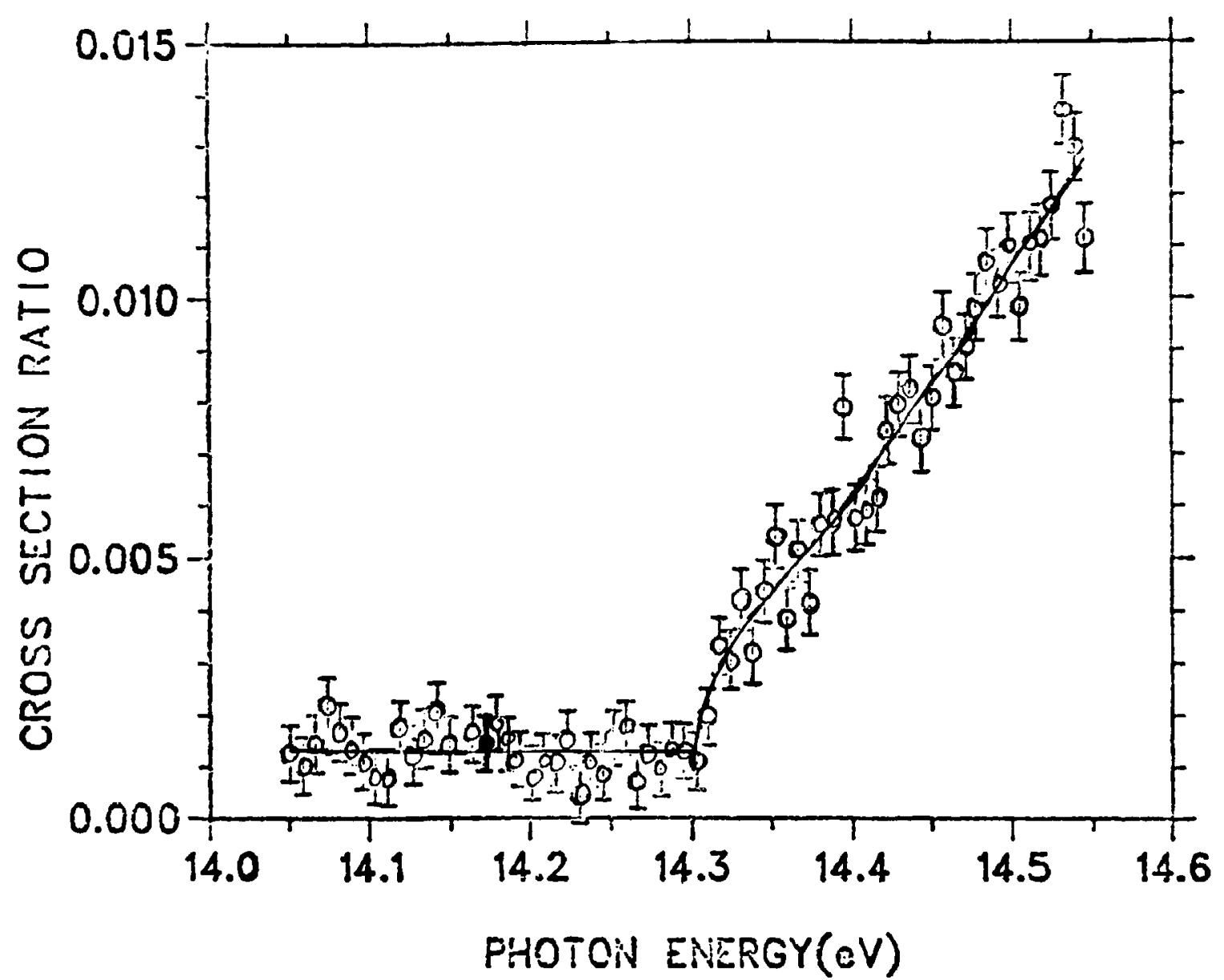

Figure 55. Curve Fit with Separate Exponents in the Two Regions 
$N_{1}$ are quite large due to the poor statistics and the limited number of data points available between 14.30 and $14.35 \mathrm{eV}$. Closer examination of figure 55 indicates a sharp step at the anomalous threshold followed by a slower rise to the two-electron threshold. This sharp step is the primary factor which lowers the exponent in the region below $14.35 \mathrm{eV}$. Fitting only the data below the twoelectron threshold to a single power law gives exponents of $0.64 \pm .31$ and $0.70 \pm .24$ for the $\mathrm{H}^{+}$channel and $\left(\mathrm{H}^{+} / \mathrm{H}^{\circ}\right)$ channel respectively. We have only 7 data points with poor counting statistics in this region. It is hard to draw conclusions about the shape of the curve below threshold. It does appear that a separate threshold law with an exponent lower than one is at work in this region. The data of the fine scan over the threshold was compared to the modulated linear law predicted by Temkin and Hahn (1974) who suggest a threshold law of the form

$$
\sigma \sim\left(1+B \sin \left[F \ln \left(E-E_{O}\right)+C\right]\right)\left(E-E_{O}\right)
$$

where $\left(E-E_{O}\right)$ is the photon energy above threshold and $B$ gives the amplitude of the modulations. As B approaches zero the expression reduces to a linear law. F determines the frequency of the oscillations which is not constant but decreases with increasing energy, while $C$ determines the phase. 
Temkin has recently written (1980) that it is quite appropriate to fit this form over a constant background above threshold due to the field stripping phenomenon. Points above threshold from the fine scan were fit with the form

$$
\sigma=D+A\left[1+B \sin \left(F \ln \left[E-E_{o}\right]+C\right)\right]\left[E-E_{o}\right]
$$

where $D$ is the constant background due to field stripping and $\mathrm{A}$ is an arbitrary scale factor. Note that this is exactly the same data set which was fit by a power law (see Figure 53 and 54) for points above threshold.

For all these fits $E_{0}$ was fixed at $14.35 \mathrm{eV}$ while all other parameters were allowed to be free. Figures 56 through 58 show typical results. Notice the differences between the three figures. Figure 56 shows slight ascillations. Figure 57 looks identical to the fit to a power law with a smoothly rising curve, while figure 58 shows pronounced oscillations.

Table 6, which gives the parameters from fitting, reflects these differences. The parameters $A$ and $D$ are very close to the scale and background parameters obtained in fitting with a power law. The other parameters are not well characterized by the fitting routine. Very high correlations are seen on these parameters, particularly on F. The three curves presented here are typical of a large 
Table 6. Typical Results of Fitting Temkin's Form.

\begin{tabular}{|c|c|c|c|c|c|c|c|c|c|c|}
\hline Figure & File & Detector & A & B & $\mathrm{C}$ & & D & $\mathbf{E}$ & $F$ & $x^{2}$ \\
\hline 55 & $22 F 75$ & $\mathrm{H}^{+}$ & $165 \pm 8$ & $.07 \pm .04$ & $.8 \pm 2$ & 16.1 & \pm .9 & $\begin{array}{c}\text { Fixed } \\
14.35 \mathrm{eV}\end{array}$ & $8 \pm 1$ & $44 / 24$ \\
\hline 56 & 22576 & $\mathrm{H}^{+} / \mathrm{H}^{\circ}$ & $.0055 \pm .0008$ & $-6.1 \pm .6$ & $-.4 \pm .7$ & .0048 & \pm .0004 & $\begin{array}{l}\text { Fixed } \\
14.35 \mathrm{eV}\end{array}$ & $6.8 \pm .3$ & $40 / 24$ \\
\hline 57 & $22 F 78$ & $\mathrm{H}^{+} / \mathrm{H}^{\circ}$ & $.042 \pm .002$ & $-.13 \pm .04$ & $6 \pm 1$ & .0042 & \pm .0004 & $\begin{array}{c}\text { Fixed } \\
14.35 \mathrm{eV}\end{array}$ & $23.0 \pm .5$ & $30 / 24$ \\
\hline
\end{tabular}




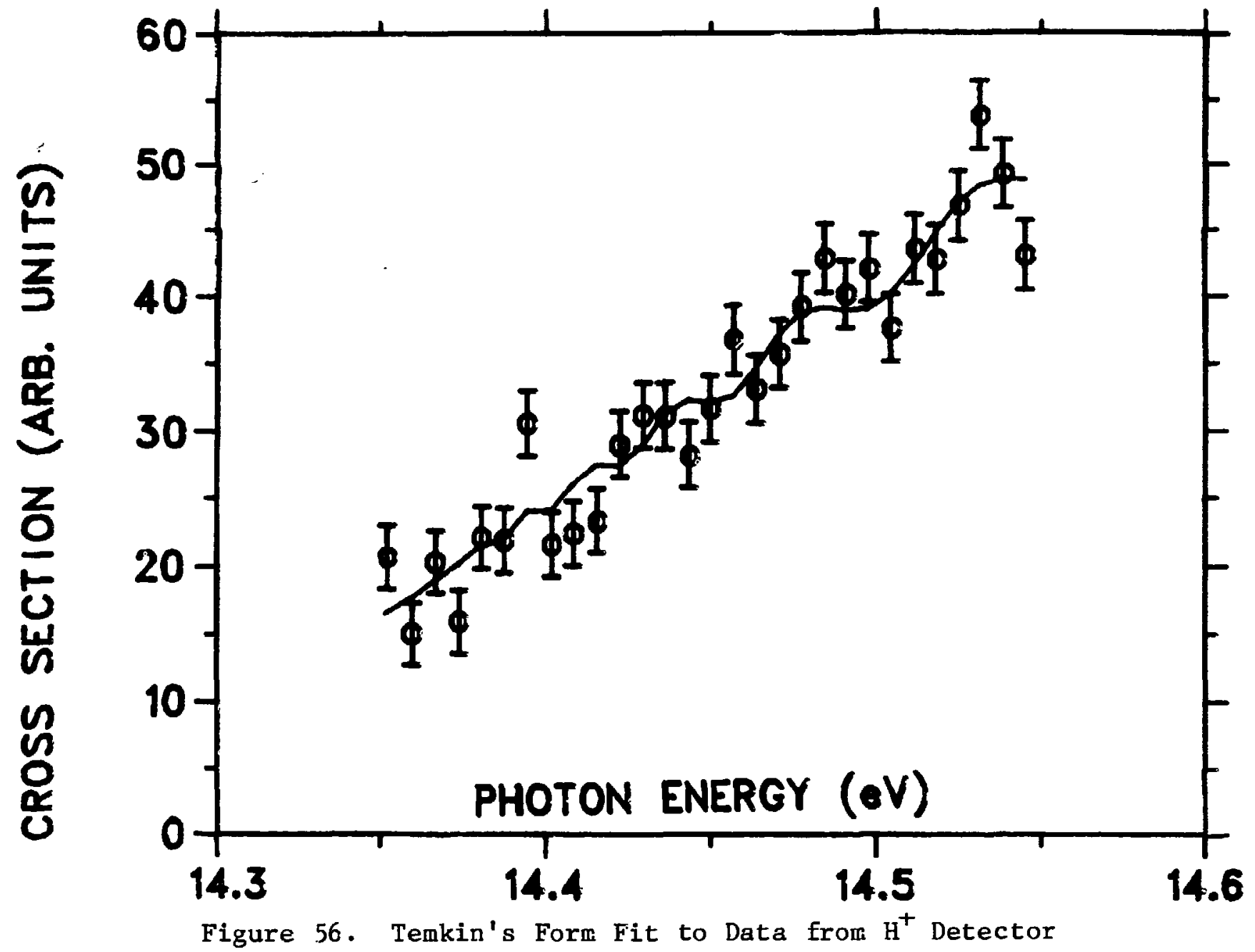




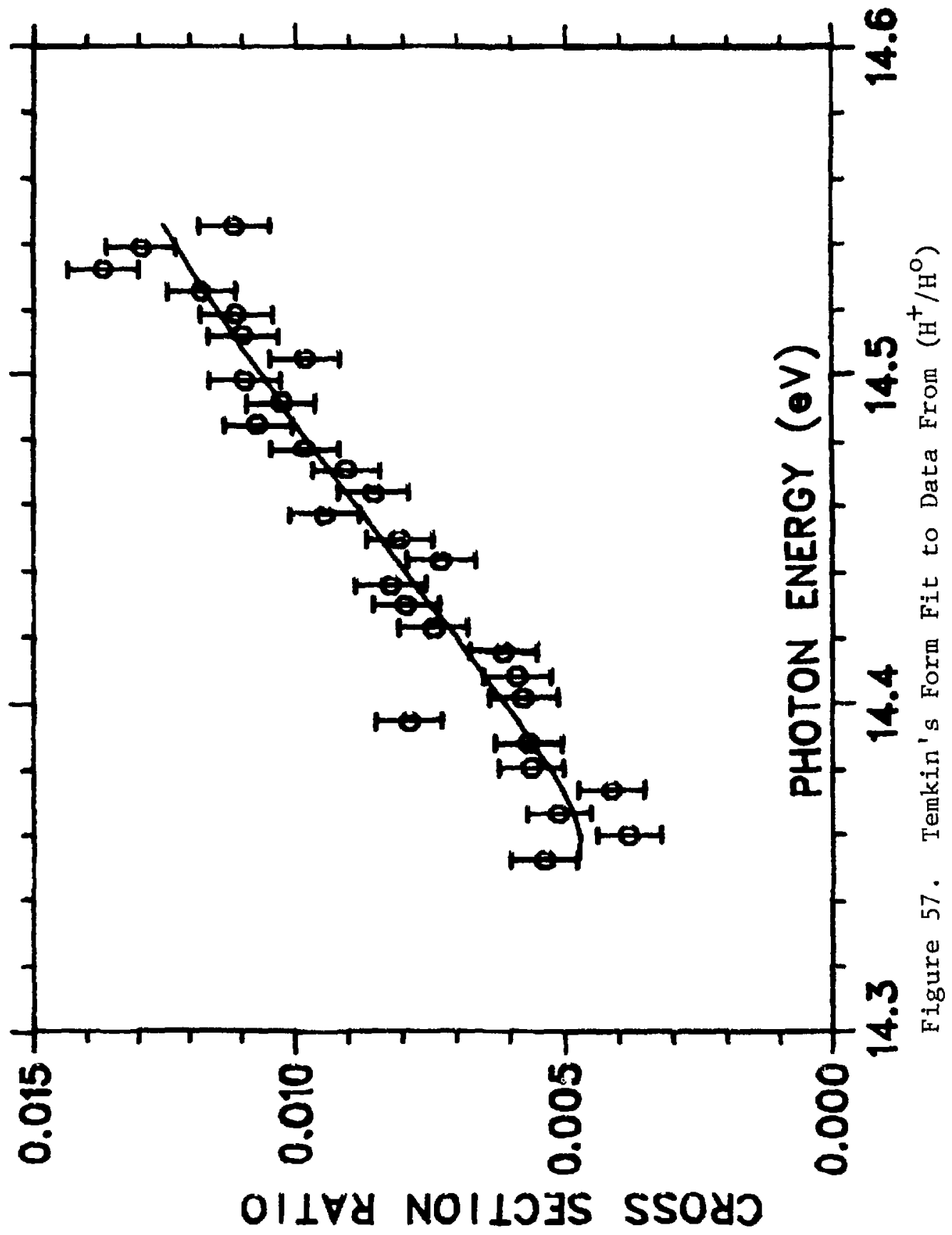




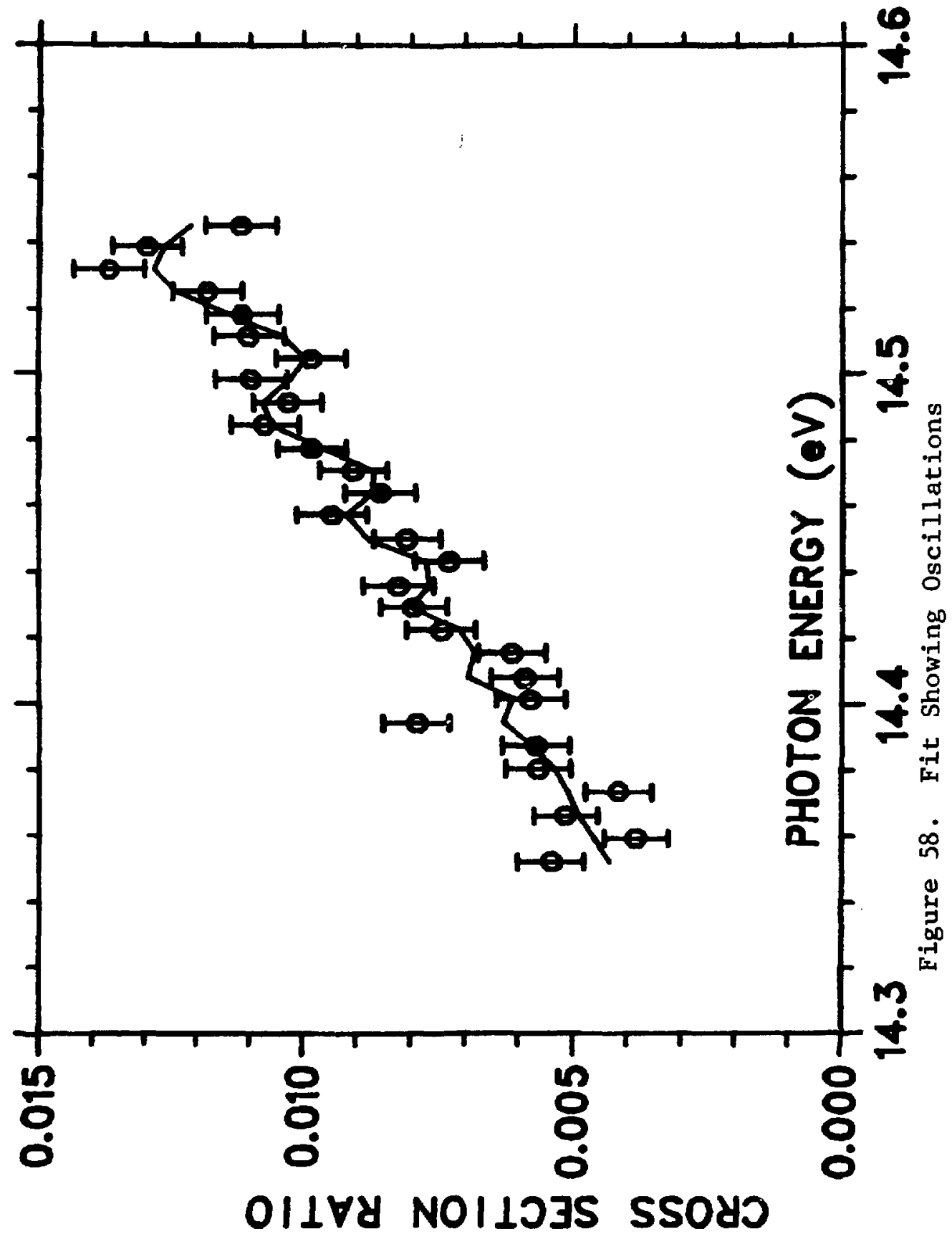


number of fitting attempts. A wide assortment of curves with various oscillation frequencies were obtained depending primarily on the starting value given to the parameters.

This wide assortment of curves showed two general characteristics: (1) the maximum amplitude of the oscillations was less than $15 \%$ and (2) $x^{2}$ was not significantly smaller than for the equivalent fits to a power law.

The curve fitting routine has trouble fitting Temkin's form due to its extremely general nature. The large nuber of undetermined coefficients inside nested transcendental functions result in a function which can apparently take on any shape including that resulting from random fluctuations in the data. The nature of the curve found by MINUIT is largely a matter of the starting values of the parameters $C, B$, and particularly F.

There are any number of combinations of the parameter values which will give the same general shape to the curve. The difference is seen only in the nature cf the small oscillations which are below the noise level. In fact the parameters may be specified so as to give a threshold law which is experimentaly indistinguishable from the Wannier's law.

While the current data cannot rule out the possible existence of the predicted oscillation. It is clear that 
they are not a dominant feature. The power law seems to be a more reasonable description since it is simple and more explicit in successfully predicting the observed behavior. It is however doubtful that any experimental measurement could deny the existence of the predicted oscillation given the highly flexible form of the law. 


\section{v}

\section{CONCLUSION}

The Doppler shifted laser relativistic ion crossed beam technique has been successfully extended to measurements of the threshold behavior for one and two electron photodetachment from the $\mathrm{H}^{-}$ion with excellent results. Two-electron photodetachment has been observed for the first time and the cross section for the process has been measured from threshold to $15.5 \mathrm{eV}$. The one-electron photodetachment cross section was simultaneously measured over tise same energy range allowing normalization of the two-electron data to the calculated one-electron cross section.

The measured value agrees in shape with the 1976 calculation of Broad and Reinhardt. It is, however, higher by a factor of 3 than the calculated value. This factor is within the combined uncertainity of measurement and the calculation.

The two-electron threshold region was examined in detail. The threshold cross section was fitted with a 
power law of the form o $\sim\left(h_{v}-E_{0}\right)^{N} . N$ is found to be $1.03 \pm .15$ or $1.10+.21$ depending on the method of analysis employed. This is consistent with either the Wannier law or a linear threshold law. The threshold data were also fitted with Temkin's modulated linear law and limits were placed on the maximum amplitude of the predicted osicllations.

The single-electron photodetachment cross section was measured from threshold at to $15.6 \mathrm{eV}$. The cross section was normalized to the calculation of Broad and Reinhardt and found to agree qualitatively with the theory. These data were also compared to the model of Armstrong with good agreement.

The threshold behavior for one-electron photodetachment was examined in detail and found to be consistent with the Wigner threshold theory as extended by Branscomb. The range of validity of the $3 / 2$ power law was examined. The electron affinity of hydrogen was determined to be $0.753 \pm 0.005 \mathrm{eV}$. This is the most accurate measurement of the quantity and is closer to the theoretical value than any previous measurements.

Partial cross sections into excited states of $\mathrm{H}^{\circ}$ were measured using the fleld ionization effect. The threshold for excitation into the $H^{\circ}(n=5)$ state was clearly observed. 
Thresholds for channels into $\mathrm{H}^{\circ}$ states with $\mathrm{n}=4$ through 10 states were also seen.

A series of resonances below the $n=6$ threshold was observed for the first time. These resonances were seen as dips in the partial cross section into $\mathrm{H}^{\circ}(\mathrm{n}=5)$. The resonances were completely obscured by the continuum in the total cross section and thus could only be observed in the partial cross section. 


\section{ACKNOWLEDGEME.NTS}

It would be impossible to give proper thanks to all of those who have contributed directly or indirectly to the contents of this thesis. By its very nature experimental physics at a large accelerator is a team effort, and the atomic physics group at LAMPF is no exception.

I wish to express my deepest appreciation to major advisor Professor Howard Bryant, who suggestd this experimental problem and provided guidance, encouragement, and support throughout the course of the work.

I would also like to thank $\mathrm{Dr}$. Joey Donahue for his inspiration in conceiving the original concept of the experiment and providing advice on design of the apparatus. The role of Professor David Clark also cannot be overstated. He was responsible for most of the mechanical and electrical design of the experiment as well as all computer software.

I wish to thank the other members of my committee as well, Professor Christopher Leavitt and Dr. Gary Tisone, for their constructive criticisms and questions that contributed to a better understanding of the experiment. I am also grateful to Ken Butterfield for the skillful preparation of many of the computer graphs presented here. Acknowledgement with thanks is also due numerous other experimenters, including Drs. P. A. M. Gram, R. H. Hamm, M. E. Hamm, and Professor W. W. Smith. 


\section{List of References}

Aashamar, K., Nucl. Instrum. Methods 90, 263 (1970).

Ajmera, M. P., and K. T. Chung, Phys. Rev. A 12, 475 (1975).

Armstrong, B.A. Phys. Rev. 131, 1132 (1963).

Barret, R. J. et al., Nucl. Instrum. Methods 129, 441 (1975).

Bartlett, J. H., Phys. Rev. 51, 661 (1937).

Bates, Fundaminksy, Massey, and Leich, Trans. Roy. Soc. (London) 243, 93 (1950).

Bayfield, J. E., Review Section of Physics Letters 51, No. $6,317-391$ (1979).

Bevington, P., Data Reduction and Error Analysis for the Physical Sciences (McGraw-Hill, New York, 1969) Table

Berry, R. S., Chem. Rev. 69, 533 (1969).

Bethe, H., Z. Physik 57, 815 (1929).

Branscomb, L. M., and J. J. Smith, Phys. Rev. 98, 1028 (1955).

Branscomb, L. M., and B. E. J. Pagel, Monthly Nat. Reg. Astron. Soc. 118, 258 (1958).

Broad, J. T. and W. P. Reinhardt, Phys. Rev. A 14, 6, 2159 (1976).

Bryant, H. C., J. Donahue, H. Sharifian, H. Tootonchi, P. A. M. Gram, J. Pratt, and M. Yates, Phys. Rev. Lett. 38, 228 (1977).

Bryant, H. C., D. Clark, J. Donahue, C. Frost, K. Butterfield, M. Hamm, R. Hamm, W. Smith, Atomic Physics with Relativistic Beams, Seventh, ICPEAC (1980) to be published in Conference Proceedings.

Bryant, H. C., P. A. Lovoi, and G. G. Ohlsen, Phys. Rev. Lett. 27,1628 (1971). 
Chandrasekhar, S., Astrophys, 3. 102, 223 (1945), 102, 395 (1945).

Chandrasekhar, S., Astrophys, J. 128, 114 (1958).

Chandrasekhar, S., and Elbert, D., Astrophys, J. 128, 633 (1958).

Clark, D. A., C. A. Frost, H. C. Bryant, J. B. Donahue, D. W. Hudgings, M. McNaugaton, and M. A. Yates, IEEE Nuc1. Sci. NS-26\#3, 3291 (1979).

Cvejanovic, S., and F. H. Read, J. Phys. B7, 1841 (1974).

Donahue, J. B., Conference on the Application of Accelerators in Research and Industry, Denton TX (to be published in IEEE Trans. Nucl. Sci. April 1981).

Fano, V., J. Phys. B. Vol. 7, No. 14 (1974).

Feldmann, D., Z. Naturforsch 25a, 621 (1970).

Frost, C. A., H. C. Bryant, D. A. Clark, J. B. Donahue, P. A. M. Gram, R. H. Hamm, and W. W. Smith, Bul. Amer. Phys. Soc. (1980).

Gallagher, T. F., et al., Phys. Rev. A 17, 904 (1978).

Geltman, S., Astrophys, J. 136, 935 (1962).

Grujic, P., J. Phys. B5, L137 (1972).

Hamm, M. E., H. C. Bryant, D. A. Clark, J. B. Donahue, C. A. Frost, P. A. M. Gram, R. H. Hamm, and W. W. Smith, Phys. Rev. Lett. 43, 23, 1715 (1979).

Hylleraas, E. A., Z. Physik 63, 291 (1930).

Hyman, H. A., V. L. Jacobs, and P. G. Burke, J. Phys. B5, 2282 (1972).

James, F., and M. Roos, Computer Physics Communications 10, 343-367 (1975).

John, T., Astrophys, J. 131, 743 (1960).

Kang, I., Phys. Lett. 31A, 172 (1970).

Khvostenko, V. I., Sov. Phy. - JETP 10, 465 (1960). 
Landau, L., Journal of Physics U.S.S.R. $\underline{8}, 201$ (1944).

Lineberger, W. C., Phys. Rev. Lett. 25, 424 (1970).

Lineberger, W. C., Personal Communication, September 1980 .

Macek, J., Proc. Phys. Soc. (Lond.) 92, 365 (1967). Marchand, D. 123 C. Paquet, and P. Marnet, Phys. Rev. 180 ,

Pekeris, Phys. Rev. 112, 1649 (1958).

Peterkorp, R. JETP 31, 374 (1970).

Peterkorp, R. and A. Liepinsh, Sixth ICPEAC (Cambridge, USA: MIT Press), 212 (1969).

Popp, H. P., and S. Kruse, J. Quant. Spectrosc. Radiant Transfer, Vol. 16, 683-688 (1976).

Rau, A. R., Phys. Rev. A4, 207 (1971).

Reinhardt, W. R., Personal Communication, July 1980.

Risley, J., Atomic Physics IV, G. Zu Putlitz, E. Weber, A. Winnacker, Plenum Press, New York (1975).

Rudge, M. and M. Seaton, Proc. Phys. Soc. A283, 262 (1965).

Sharifian, Ph. D. Dissertation, University of New Mexico, 1977 .

Slater II, J. M., Ph.D. Dissertation, University of Colorado, 1976.

Smith, S. J., and P. S. Burch, Phys. Rev. 116, 1125 (1959).

Temkin, A., and Y. Hahn, Phys. Rev. A9, 708 (1974).

Temkin, A., Phys. Rev. A 22, 1 (1980).

Temkin, A., Phys. Rev. 176, 80 (1968).

Temkin, A., Phys. Rev. Lett. 16, 835 (1966).

Temkin, A., Personal Communication, May 1980.

Tootoonchi, C. H., Ph.D. Dissertation, University of New Mexico, 1977 . 
Vinkalns, I. and M. Gailitis, Fifth ICPEAC (Leningrad: NAUKA), 648 (1967).

Wannier, G. H., Phys. Rev. 90, 5, 817 (1953).

Weisner, J. D., and B. H. Armstrong, Proc. Phys. Soc. (London) 83, 31 (1964).

Wigner, E. P., Phys. Rev. 73, 9, 1002 (1948).

Wildt, K., Astrophys, J. 89, 295 (1939).

Woodruff, P. R., and J. A. R. Samson, Phys. Rev. Lett. 45, 2, 110 (1980). 